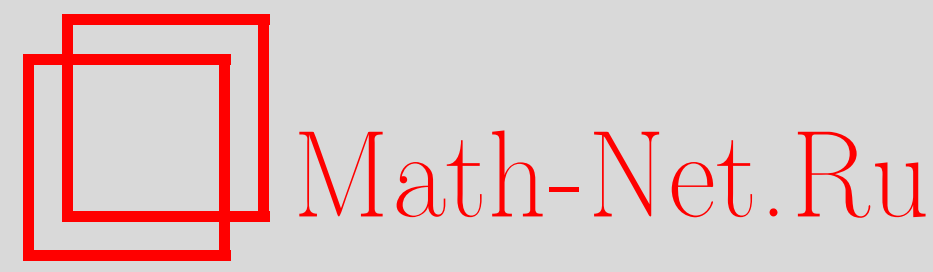

Ю. Ф. Коробейник, Представляющие системы экспонент и задача Коши для уравнений в частных производных с постоянными коэффициентами, Изв. РАН. Сер. матем., 1997, том 61, выпуск 3, 91-132

DOI: https://doi.org/10.4213/im127

Использование Общероссийского математического портала Math-Net.Ru подразумевает, что вы прочитали и согласны с пользовательским соглашением

http://www.mathnet.ru/rus/agreement

Параметры загрузки:

IP: 34.227 .88 .159

26 апреля 2023 г., 16:28:41 
УДК 517.95

\author{
Ю. Ф. Коробейник
}

\title{
Представляющие системы экспонент и задача Коши для уравнений в частных производных с постоянными коэффициентами
}

Рассматривается задача Коши по $z_{2}$ для однородного линейного уравнения в частных производных с постоянными коэффициентами и двумя независимыми переменными $z_{1}, z_{2} \in \mathbb{C}$. Показывается, что характер сравнительной гладкости по $z_{1}$ и $z_{2}$ аналитических и ультродифференцируемых решений задачи Коши существенно зависит от величины $\rho_{2}$ и, как правило, полностью ею определяется. Получены также довольно общие теоремы единственности и указаны условия, при выполнении которых построенное частное решение зависит от начальных функций и непрерывно и линейно.

Библиография: 38 наименований.

\section{§1. Постановка задачи}

Рассмотрим уравнение в частных производных с двумя независимыми комплексными переменными

$$
M w:=\sum_{j=0}^{m} \sum_{s=0}^{l_{j}} a_{s, j} \frac{\partial^{s+j} w\left(z_{1}, z_{2}\right)}{\partial z_{1}^{s} \partial z_{2}^{j}}=0 .
$$

Здесь $a_{s, j} \in \mathbb{C}, 0 \leqslant l_{j}<+\infty$ и суммирование ведется по тем $j \leqslant m$, для которых $a_{l_{j}, j} \neq 0$ (т.е. по тем $j$, для которых $a_{s, j} \neq 0$ хотя бы для одного номера $s \geqslant 0$ ). При этом $a_{l_{m}, m} \neq 0$.

Пусть при $l=1,2 \mathscr{F}_{l}$ - плотное в себе подмножество $\mathbb{C}$ и $C^{\infty}\left(\mathscr{F}_{l}\right)$-пространство всех комплекснозначных определенных на $\mathscr{F}_{l}$ функций, имеющих производные всех порядков. При этом функция $y(z)$ дифференцируема в точке $z_{0} \in \mathscr{F} l$, если существует конечный предел $\lim _{z \in \mathscr{F}_{l}} \frac{y(z)-y\left(z_{0}\right)}{z-z_{0}}$. Всюду далее $0 \in \mathscr{F}_{2}$.

Пусть при тех же $l \mathscr{E}_{l}\left(\mathscr{F}_{l}\right)$ - полное отделимое локально вьпуклое пространство (ПОЛВП) такое, что $\mathscr{E}_{l}\left(\mathscr{F}_{l}\right) \subseteq C^{\infty}\left(\mathscr{F}_{l}\right)$, и если $y_{n} \in \mathscr{E}_{l}\left(\mathscr{F}_{l}\right) \forall n \geqslant 1, y \in \mathscr{E}_{l}\left(\mathscr{F}_{l}\right)$, $y_{n} \rightarrow y$ при $n \rightarrow \infty$ в $\mathscr{E}_{l}\left(\mathscr{F}_{l}\right)$, то $\forall s \geqslant 0$ последовательность $\left\{y_{n}^{(s)}(z)\right\}_{n=0}^{\infty}$ сходится к $y^{(s)}(z)$ равномерно на каждом компакте из $\mathscr{F} l$.

Обозначим символом $\left(\mathscr{E}_{1}\left(\mathscr{F}_{1}\right) ; \mathscr{E}_{2}\left(\mathscr{F}_{2}\right)\right)$ множество функций $u\left(z_{1}, z_{2}\right)$, определенных на $\mathscr{F}_{1} \times \mathscr{F}_{2}$ и таких, что $\forall z_{1} \in \mathscr{F}_{1} u\left(z_{1}, z_{2}\right) \in \mathscr{E}_{2}\left(\mathscr{F}_{2}\right)$ (как функция от $\left.z_{2}\right)$ и $\forall z_{2} \in \mathscr{F}_{2} u\left(z_{1}, z_{2}\right) \in \mathscr{E}_{1}\left(\mathscr{F}_{1}\right)$ (как функция от $\left.z_{1}\right)$.

Работа выполнена при финансовой поддержке Российского фонда фундаментальных исследований (коды проектов № 93-011-242 и 96-01-01041).

(C) Ю.Ф. Коровейник 1997 
Для уравнения (1) ставится задача Коши (з.К.) по $z_{2}$ : найти решение $w\left(z_{1}, z_{2}\right)$ уравнения $(1)$ из $\left(\mathscr{E}_{1}\left(\mathscr{F}_{1}\right) ; \mathscr{E}_{2}\left(\mathscr{F}_{2}\right)\right)$ такое, что

$$
\left.\frac{\partial^{k} w\left(z_{1}, z_{2}\right)}{\partial z_{2}^{k}}\right|_{z_{2}=0}=\varphi_{k}\left(z_{1}\right), \quad k=0,1, \ldots, m-1
$$

где $\varphi_{k}\left(z_{1}\right)$ - заданные функции из $\mathscr{E}_{1}\left(\mathscr{F}_{1}\right)$.

Введем характеристику уравнения $(1)$, которая является основной для всего последующего изложения. Именно, назовем коррелирующим индексом (кор. инд.) уравнения (1) по переменной $z_{2}$ величину

$$
\rho_{2}=\rho\left(z_{2}\right):=\max \left\{\frac{l_{j}-l_{m}}{m-j}: 0 \leqslant j<m, a_{l_{j}, j} \neq 0\right\} .
$$

Образуем еще множества

$$
\begin{aligned}
& \mathscr{N}_{0}=\left\{j: 0 \leqslant j<m ; a_{l_{j}, j} \neq 0 ; l_{j}-l_{m}=\rho_{2}(m-j)\right\}, \quad \mathscr{N}_{2}=\{m\} \cup \mathscr{N}_{0} \\
& \mathscr{N}_{1}=\left\{j: 0 \leqslant j<m ; a_{l_{j}, j} \neq 0 ; l_{j}-l_{m}<\rho_{2}(m-j)\right\}
\end{aligned}
$$

Заметим, что $\rho_{2} \leqslant 1 \Longleftrightarrow l_{j}+j \leqslant l_{m}+m$ для всех $j, 0 \leqslant j \leqslant m$, для которых $a_{l_{j}, j} \neq 0$. В частности, для уравнения

$$
\frac{\partial^{m} w\left(z_{1}, z_{2}\right)}{\partial z_{2}^{m}}+\sum_{j=0}^{m-1} \sum_{s=0}^{l_{j}} a_{s, j} \frac{\partial^{s+j} w\left(z_{1}, z_{2}\right)}{\partial z_{1}^{s} \partial z_{2}^{j}}=0
$$

условие $\rho_{2} \leqslant 1$ равносильно тому, что $l_{j}+j \leqslant m$ для всех $j \leqslant m-1$. Иначе говоря, для уравнения (3) $\rho_{2} \leqslant 1$ тогда и только тогда, когда оно является уравнением класса Ковалевской (см. [1, с. 286]). Будем говорить, что уравнение (1) принадлежит обобщенному классу Ковалевской, если $\rho_{2} \leqslant 1$, т.е. если $l_{m}+m \geqslant l_{j}+j$ для всех $j \leqslant m-1$ таких, что $a_{l_{j}, j} \neq 0$.

В настоящей работе с помощью рядов экспонент строится частное решение з.К. $(1),(2)$ и показывается, что характер гладкости этого решения по переменным $z_{1}$ и $z_{2}$ существенно зависит от величины кор. инд. $\rho_{2}$. При этом, что весьма важно, условие $l_{m}=0$ не предполагается.

В следующем параграфе излагаются необходимые для дальнейшего сведения об асимптотическом поведении ветвей алгебраической функции, определяемой уравнением $Q(x, y)=0$, где $Q(x, y):=\sum_{j=0}^{m} y^{j} \sum_{s=0}^{l_{j}} a_{s, j} x^{s}-$ характеристический многочлен уравнения (1). Метод, применяемьй в настоящей статье, описывается в $\S 3$, и там же получены в самой общей ситуации оценки коэффищиентов представления решения з.К. (1), (2) в виде ряда экспонент, через коэффициенты разложений начальных функций. В $\S 4-7$ и $\S 9$ изучаются свойства (характер гладкости) частного (представленного в виде ряда экспонент) решения з.К. (1), (2) при различных значениях кор. инд. $\rho_{2}$. В $\S 8$ излагаются некоторые дополнительные сведения о кор. индексах, необходимые для рассмотрения случая $\rho_{2}>1$. В $\S 10$ исследуются вопросы сушествования решения з.К. (1), (2), зависяшего от начальных данных непрерывно, а в $§ 11$ - непрерывно и линейно. 


\section{§ 2. Некоторые сведения о характеристическом многочлене}

Пусть $Q(\lambda, \mu)=Q_{1}^{r_{1}}(\lambda, \mu) \ldots Q_{q}^{r_{q}}(\lambda, \mu)$ - разложение характеристического многочлена $Q(\lambda, \mu)$ на неприводимые многочлены $Q_{s}$. В дальнейшем предполагается, что ни один из многочленов $Q_{s}$ не вырождается в многочлен от одной переменной $(\lambda$ или $\mu)$. Каждое уравнение $Q_{s}^{r_{s}}(\lambda, \mu)=0$ в окрестности бесконечно удаленной точки определяет $n_{s}$ различных ветвей $\mu_{j, s}(\lambda)$ кратности $r_{s}$ соответствуюшей алгебраической функции, причем $\sum_{s=1}^{q} n_{s} r_{s}=m$, и при $|\lambda| \geqslant R_{0}$ $\mu_{j, s}(\lambda)=\sum_{k=\alpha}^{\infty} c_{j, s, k} \lambda^{-\frac{k}{\nu}}$, где $\nu$ - натуральное число, $\alpha$-целое число, $\nu=\nu(j, s)$, $\alpha=\alpha(j, s)$ (см., например, [2, c. 535-537]). Отсюда легко получить, что если $\mu_{j}(\lambda)$ и $\mu_{s}(\lambda)$ - две различные ветви, соответствуюшие одному многочлену $Q_{r}$ или разным многочленам $Q_{m_{1}}, Q_{m_{2}}$, то найдутся натуральное число $M$ и число $d>0$ такие, что при $|\lambda| \geqslant R_{1}$

$$
\left|\mu_{j}(\lambda)-\mu_{s}(\lambda)\right| \geqslant d|\lambda|^{-M} .
$$

Пусть $\mu(\lambda)$ - любая из ветвей алгебраической функции, соответствующей какому-либо неприводимому многочлену $Q_{s}$. Как показано в $[3, \S 2]$ и в $[4, \S 2]$, всегда сушествует конечный предел $\tau_{\mu}=\lim _{\lambda \rightarrow \infty} \mu(\lambda) \lambda^{-\rho_{2}}$. При этом число $\tau_{\mu}$ является одним из корней многочлена $P(x):=\sum_{k \in \mathcal{N}_{2}} a_{l_{k}, k} x^{k}$. Разньгм ветвям $\mu$, вообще говоря, не обязательно соответствуют разные $\tau_{\mu}$.

Положим $\alpha_{\mu}(\lambda)=\mu(\lambda)-\lambda^{\rho_{2}} \tau_{\mu}$. Очевидно, что $\lim _{\lambda \rightarrow \infty} \alpha_{\mu}(\lambda) \lambda^{-\rho_{2}}=0$, и если $\rho_{2} \leqslant 0$, то $\lim _{\lambda \rightarrow \infty} \alpha_{\mu}(\lambda)=0$. Пусть теперь $\rho_{2}>0$. Будем говорить, что уравнение $Q(\lambda, \mu)=0$ допускает сильную асимптотику (по $\lambda$ ), если любая ветвь $\mu(\lambda)$ имеет представление $\mu(\lambda)=\lambda^{\rho_{2}} \tau_{\mu}+\alpha_{\mu}(\lambda)$, где $\sup _{|\lambda| \geqslant R_{0}}\left|\alpha_{\mu}(\lambda)\right|<\infty$. В работах $[3, \S 2]$ и $[4, \S 2]$ получены следуюшие достаточные условия наличия сильной асимптотики по $\lambda$.

Предположим, что выполняются два условия:

a) все корни многочлена $P(x)$ простые;

b) $0<\rho_{2} \leqslant 1$;

а также одно из таких двух:

$\left.\mathrm{c}_{1}\right) \forall j \in \mathscr{N}_{1}:\left(l_{j}-l_{m}\right) \leqslant \rho_{2}(m-j-1)$ или

$\left.\mathrm{c}_{2}\right) \delta k_{0} \geqslant \rho_{2}$, где $k_{0}=m-\max \left\{l: l \in \mathscr{N}_{1}\right\}$, а $\delta=\rho_{2}-\max \left\{\left(l_{j}-l_{m}\right) /(m-j):\right.$ $\left.j \in \mathscr{N}_{1}\right\}$.

Тогда уравнение $Q(\lambda, \mu)=0$ допускает сильную асимптотику по $\lambda$ (см. [3], [4]).

Заметим, что если $\rho_{2}=\frac{1}{M}$, где $M=1,2, \ldots$, то условие $\left.c_{1}\right)$ выполняется автоматически: если бы оно было нарушено, то $\forall k \in \mathscr{N}_{1} M\left(l_{k}-l_{m}\right)<(m-k)$ и $\exists k_{0} \in \mathscr{N}_{1} M\left(l_{k_{0}}-l_{m}\right)>\left(m-k_{0}-1\right)$, откуда $m-k_{0}-1<M\left(l_{k_{0}}-l_{m}\right)<m-k_{0}$, что невозможно. В частности, если $\rho_{2}=1$ и все корни многочлена $P(x)$ простые, то уравнение $Q(\lambda, \mu)=0$ имеет сильную асимптотику по $\lambda$.

В работах [3] и [4] приведено также достаточное условие наличия сильной асимптотики для случая, когда $P(x)$ может иметь и кратные корни.

\section{§ 3. Формальное построение решения задачи Коши и оценка коэффициентов из его представления}

Всюду далее предполагается, что пространства $\mathscr{E}_{j}\left(\mathscr{F}_{j}\right), j=1,2$, кроме указанных выше свойств, обладают еще инвариантностью относительно дифференци- 
рования в том смысле, что оператор дифференцирования непрерывен в каждом из этих пространств.

Далее, предполагается, что множества $\Lambda_{j}:=\left\{\alpha \in \mathbb{C}: \exp \alpha z_{j} \in \mathscr{E}_{j}\left(\mathscr{F}_{j}\right)\right\}$ непусты при $j=1,2$.

Наконец, считаем, что пространство $\mathscr{E}_{1}\left(\mathscr{F}_{1}\right)$ имеет хотя бы одну абсолютно представляющую систему вида $\mathscr{E}_{\Lambda}:=\left(\exp \lambda_{k} z_{1}\right)_{k=1}^{\infty}, \lambda_{k} \in \Lambda_{1}, k=1,2, \ldots$, и $\lim _{k \rightarrow \infty} \lambda_{k}=\infty$.

Напомним [5], что последовательность элементов $\left(x_{k}\right)_{k=1}^{\infty}$ отделимого локально выпуклого пространства $H$ с набором преднорм $\mathscr{P}=\{p\}$ называется абсолютно представляющей системой (АПС) в $H$, если любой элемент $x$ из $H$ можно представить в виде ряда $x=\sum_{k=1}^{\infty} c_{k} x_{k}$, абсолютно сходящегося в $H$ :

$$
\sum_{k=1}^{\infty}\left|c_{k}\right| p\left(x_{k}\right)<\infty \quad \forall p \in \mathscr{P} .
$$

Будем считать, что все показатели $\lambda_{k}$ достаточно велики по модулю: $\left|\lambda_{k}\right| \geqslant R_{0}$, где число $R_{0}$ взято настолько большим, что в области $|\lambda| \geqslant R_{0}$ определены все описанные выше ветви $\left(\mu_{j}(\lambda)\right)_{j=1}^{N}$, порожденные уравнением $Q(\lambda, \mu)=0$.

Пусть $p_{j}$ - кратность ветви $\mu_{j}(\lambda)$. Тогда $\sum_{j=1}^{N} p_{j}=m$. Имеем $Q(\lambda, \mu)=$ $\sum_{k=0}^{m} \mu^{k} R_{k}(\lambda)$, где $R_{k}$ - многочлен степени $l_{k}, k=1,2, \ldots, m-1: R_{k}(\lambda)=$ $\sum_{s=0}^{l_{k}} a_{s, k} \lambda^{s}$.

Уравнение (1) обладает хорошо известным набором частных решений, играющих важную роль в дальнейшем изложении. Именно, если $\lambda_{0}-$ произвольное зафиксированное комплексное число, а $\mu$-корень уравнения $Q\left(\lambda_{0}, \mu\right)=0$ кратности $l$, то функция $z_{2}^{s} \exp \left(\lambda_{0} z_{1}+\mu z_{2}\right)$ удовлетворяет уравнению $(1)$ в $\mathbb{C}^{2}$, если $s \leqslant l-1$. Выберем последовательность $\left(\lambda_{k}\right)_{k=1}^{\infty}$, где $\lambda_{k} \rightarrow \infty$, и $R_{m}\left(\lambda_{k}\right) \neq 0, k=1,2, \ldots$ Тогда для каждого $\lambda_{k}$ найдется $m$ корней $\mu_{j, k}$ (с учетом их кратностей) уравнения $Q\left(\lambda_{k}, \mu\right)=0$. Будем предполагать, что числа $\lambda_{k}$ достаточно велики по модулю: $\left|\lambda_{k}\right| \geqslant R_{0}$. Тогда $\mu_{j, k}=\mu_{j}\left(\lambda_{k}\right)$, где $\mu_{j}$ - одна из $N=\sum_{s=1}^{q} n_{s}$ различных ветвей кратности $p_{j}$, так что $\sum_{j=1}^{N} p_{j}=m$. Искомое решение з.К. (1), (2)будем искать в виде (пока формально записанного) ряда

$$
w_{0}\left(z_{1}, z_{2}\right)=\sum_{j=1}^{N} \sum_{s=0}^{p_{j}-1} \sum_{k=1}^{\infty} a_{j, s}^{(k)}\left(z_{2}\right)^{s} \exp \left[\lambda_{k} z_{1}+\mu_{j, k} z_{2}\right]
$$

При этом предполагается, что $\lambda_{k} \in \Lambda_{1}$ и $z_{2}^{s} \exp \mu_{j, k} z_{2} \in \mathscr{E}_{2}\left(\mathscr{F}_{2}\right) \quad \forall s \leqslant p_{j}-1$, $k \geqslant 1$. Будем считать, что каждый ряд $\sum_{k=1}^{\infty} a_{j, s}^{(k)}\left(z_{2}\right)^{s} \exp \left[\lambda_{k} z_{1}+\mu_{j, k} z_{2}\right]$ сходится абсолютно в $\mathscr{E}_{1}\left(\mathscr{F}_{1}\right)$ при любом фиксированном $z_{2} \in \mathscr{F}_{2}$ и в $\mathscr{E}_{2}\left(\mathscr{F}_{2}\right)$ при любом фиксированном $z_{1} \in \mathscr{F}_{1}$ (при $\left.s=0,1, \ldots, p_{j}-1 ; j=1,2, \ldots, N\right)$. В этом случае будем говорить, что ряд (5) сходится абсолютно в $\left(\mathscr{E}_{1}\left(\mathscr{F}_{1}\right) ; \mathscr{E}_{2}\left(\mathscr{F}_{2}\right)\right)$.

При этих предположениях, учитывая, что каждое слагаемое

$$
a_{j, s}^{(k)}\left(z_{2}\right)^{s} \exp \left[\lambda_{k} z_{1}+\mu_{j, k} z_{2}\right]
$$


удовлетворяет уравнению (1), а оператор дифференцирования непрерывен в каждом пространстве $\mathscr{E}_{l}\left(\mathscr{F}_{l}\right), l=1,2$, получаем, что функция $w_{0}$ из $\left(\mathscr{E}_{1}\left(\mathscr{F}_{1}\right) ; \mathscr{E}_{2}\left(\mathscr{F}_{2}\right)\right)$ удовлетворяет уравнению (1) на множестве $\mathscr{F}_{1} \times \mathscr{F}_{2}$. При этом $\forall z_{2} \in \mathscr{F}_{2}$, $\forall n \leqslant m-1$

$$
\frac{\partial^{n} w_{0}\left(z_{1}, z_{2}\right)}{\partial z_{2}^{n}}=\sum_{j=1}^{N} \sum_{s=0}^{p_{j}-1} \sum_{k=1}^{\infty} a_{j, s}^{(k)}\left(z_{2}^{s} \exp \mu_{j, k} z_{2}\right)^{(n)} \exp \lambda_{k} z_{1},
$$

откуда для тех же $n$ и $z_{1}$

$$
\left.\frac{\partial^{n} w_{0}\left(z_{1}, z_{2}\right)}{\partial z_{2}^{n}}\right|_{z_{2}=0}=\sum_{j=1}^{N} \sum_{s=0}^{q_{j}(n)} \sum_{k=1}^{\infty} a_{j, s}^{(k)} s ! C_{n}^{s} \mu_{j, k}^{n-s} \exp \lambda_{k} z_{1}
$$

где $\mu_{j, k}=\mu_{j}\left(\lambda_{k}\right), q_{j}(n)=\min \left\{n, p_{j}-1\right\}$.

Предположим теперь, что $\left(\exp \lambda_{k} z_{1}\right)_{k=1}^{\infty}-\mathrm{A \Pi C}$ в $\mathscr{E}_{1}\left(\mathscr{F}_{1}\right)$, и разложим начальные функции $\varphi_{n}\left(z_{1}\right)$ в абсолютно сходящиеся в $\mathscr{E}_{1}\left(\mathscr{F}_{1}\right)$ ряды:

$$
\varphi_{n}\left(z_{1}\right)=\sum_{k=1}^{\infty} b_{k, n} \exp \lambda_{k} z_{1}, \quad n=0,1, \ldots, m-1
$$

Допустим, что числа $a_{j, s}^{(k)}$ можно выбрать так, чтобы при любом фиксированном $k \geqslant 1$ они удовлетворяли соотношениям

$$
b_{k, n}=\sum_{j=1}^{N} \sum_{s=0}^{q_{j}(n)} a_{j, s}^{(k)} s ! C_{n}^{s} \mu_{j, k}^{n-s}, \quad n=0,1, \ldots, m-1
$$

Тогда $\forall n \leqslant m-\left.1 \frac{\partial^{n} w_{0}\left(z_{1}, z_{2}\right)}{\partial z_{2}^{n}}\right|_{z_{2}=0}=\varphi_{n}\left(z_{1}\right)$ и $w_{0}$ - искомое решение з.К. (1), (2).

Обоснуем прежде всего возможность указанного выбора чисел $a_{j, s}^{(k)}$. Легко проверить, что при фиксированном $k \geqslant 1$ равенства (7) образуют систему $m$ линейных уравнений относительно $m$ неизвестных $a_{j, s}^{(k)}$. Эта система уже встречалась в работе [6] при исследовании задачи Коши для аналитических решений одного частного подкласса уравнения (1).

В этой работе (см. также $[3, \S 1]$ или $[4, \S 3])$ показано, что определитель системы $(7)$ равен $\prod_{j=1}^{N}\left(1 ! 2 ! \ldots\left(p_{j}-1\right) ! \prod_{r>j}\left(\mu_{r, k}-\mu_{j, k}\right)^{p_{r} \cdot p_{j}}\right)$. По формулам Крамера имеем при $j=1,2, \ldots, N ; s=0,1, \ldots, p_{j}-1 ; k=1,2, \ldots$

$$
a_{j, s}^{(k)}=\frac{1}{\Delta_{k}} \sum_{n=0}^{m-1}(-1)^{n+\beta} b_{k, n} \Delta_{k, n}^{(\beta)}
$$

Здесь $\Delta_{k, n}^{(\beta)}$ - определитель, полученный из $\Delta_{k}$ вычеркиванием его $n$-й строки и столбца с номером $\beta$, где $\beta$ равно $s$ при $j=1$ и $\beta=s+\sum_{i=1}^{j-1} p_{i}$, если $j>1$. Учитывая, что $\forall j \leqslant N$

$$
\left|\mu_{j}(\lambda)\right|=O\left(|\lambda|^{\rho_{2}}\right)
$$


и принимая во внимание еще и оценки (4), получаем из (8) важные неравенства

$$
\left|a_{j, s}^{(k)}\right| \leqslant D\left|\lambda_{k}\right|^{T} \sum_{n=0}^{m-1}\left|b_{k, n}\right|
$$

в которых конечные числа $D, T$ от $j, s$ и $k$ не зависят. Оценки (9) позволяют во всех рассмотренных далее случаях, исходя из абсолютной сходимости в $\mathscr{E}_{1}\left(\mathscr{F}_{1}\right)$ рядов $(6)$, установить абсолютную сходимость в $\left(\mathscr{E}_{1}\left(\mathscr{F}_{1}\right) ; \mathscr{E}_{2}\left(\mathscr{F}_{2}\right)\right)$ ряда $(5)$. При этом, как будет показано ниже, характер гладкости по $z_{1}$ и по $z_{2}$ решения $w_{0}\left(z_{1}, z_{2}\right)$ будет определяться величиной кор. инд. $\rho_{2}$.

В заключение этого параграфа приведем результат, согласно которому в широком классе пространств, имеющих АПС экспонент $\left(\exp \lambda_{k} z_{1}\right)_{k=1}^{\infty}$ такую, что $\lim _{k}\left|\lambda_{k}\right|=\infty$, можно всегда считать, что $\forall k \geqslant 1\left|\lambda_{k}\right| \geqslant R_{1}$, где число $R_{1}$ можно брать как угодно большим.

ПРЕДЛОЖЕНИЕ 1. Пусть Еீ- ПОЛВП функиий одного переменного $z$, инвариантное относительно дифференчирования и имеющее хотя бы одну АПС $\left(\exp \lambda_{k} z\right)_{k=1}^{\infty}$, для которой $\lim _{k}\left|\lambda_{k}\right|=\infty$.

Тогда следующие утверждения равносильнь:

1) в $\mathscr{E}$ существует $А \Pi C\left(\exp \nu_{k} z\right)_{k=1}^{\infty}$, для которой $\lim _{k}\left|\nu_{k}\right|=\infty$, и которая остается АПС в $\mathscr{E}$ после удаления из нее любого конечного числа ее әлементов;

2) каждая $А \Pi C\left(\exp \tau_{k} z\right)_{k=1}^{\infty}$ в $\mathscr{E}$, для которой $\lim _{k}\left|\tau_{k}\right|=\infty$, остается АПС в $\mathscr{E}$ после удаления любого конечного числа ее әлементов;

3) каждьй отличный от тожсдественного нуля линейный дифференциальный оператор конечного порядка с постоянными коэффициентами сюрдективен в $\mathscr{E}$;

4) $\forall \alpha \in \mathbb{C}$ оператор $y^{\prime}-\alpha y-$ эпиморфизм $\mathscr{E}$.

ДокАЗАТЕЛЬСТво. Импликации 2) $\Longrightarrow 1), 3) \Longleftrightarrow 4$ ) очевидны, и нам остается установить соотношения 1$) \Longrightarrow 4$ ), 3) $\Longrightarrow 2$ ).

1. Пусть справедливо утверждение 3$)$, и пусть $\left(\exp \tau_{k} z\right)_{k=1}^{\infty}-$ произвольная АПС в $\mathscr{E}$ такая, что $\lim _{k}\left|\tau_{k}\right|=\infty$. Покажем, что для любого натурального $M$ последовательность $\left(\exp \tau_{k} z\right)_{k=M+1}^{\infty}$ будет АПС в $\mathscr{E}$. Положим

$$
a(z)=\prod_{k=0}^{M}\left(z-\tau_{k}\right)=\sum_{l=0}^{M} b_{l} z^{l}, \quad a(D) y=\sum_{l=0}^{M} b_{l} y^{(l)}(z)
$$

Пусть $f$ - любой элемент $\mathscr{E}$. Выберем $v \in \mathscr{E}$ так, чтобы $f=a(D) v$, и разложим $v$ в абсолютно сходящийся в $\mathscr{E}$ ряд по АПС $\left(\exp \tau_{k} z\right)_{k=1}^{\infty}: v(z)=\sum_{k=1}^{\infty} d_{k} \exp \tau_{k} z$. Отсюда

$$
f(z)=(a(D) v)(z)=\sum_{k=M+1}^{\infty} d_{k} a\left(\tau_{k}\right) \exp \tau_{k} z
$$

причем ряд сходится абсолютно в $\mathscr{E}$.

2. Пусть в $\mathscr{E}$ имеется АПС $\mathscr{E}_{\nu}\left(\exp \nu_{k} z\right)_{k=1}^{\infty}$ со свойствами, указанными в утверждении 1), и пусть $L y=y^{\prime}-\alpha y, \alpha \in \mathbb{C}$. Выберем $M$ настолько большим, чтобы 
$\left|\nu_{k}\right|>|\alpha| \forall k \geqslant M$, возьмем любой элемент $f$ из $\mathscr{E}$ и разложим его в абсолютно сходящийся в $\mathscr{E}$ ряд по АПС $\mathscr{E}_{\nu}\left(\exp \nu_{k} z\right)_{k=M+1}^{\infty}$ :

$$
f(z)=\sum_{k=M+1}^{\infty} a_{k} \exp \nu_{k} z
$$

Положим $b(\lambda)=\lambda-\alpha$ и рассмотрим ряд

$$
u(z)=\sum_{k=M+1}^{\infty} \frac{a_{k}}{b\left(\nu_{k}\right)} \exp \nu_{k} z
$$

Так как $b\left(\nu_{k}\right) \neq 0$ и $\lim _{k \rightarrow \infty}\left|b\left(\nu_{k}\right)\right|=\infty$, то этот ряд сходится абсолютно в $\mathscr{E}$, причем $u^{\prime}(z)-\alpha u(z)=f(z)$ и $L-$ эпиморфизм $\mathscr{E}$.

ЗАмЕчАнИЕ. Если пространство $\mathscr{E}$ инвариантно относительно умножения на любую экспоненту $\exp \alpha x \quad \forall \alpha \in \mathbb{C}$, то, как легко убедиться, условие 4) равносильно тому, что оператор дифференцирования сюръективен в $\mathscr{E}$.

Будем называть ряд (5), построенньй по разложениям (6) начальных функций по АПС $\mathscr{E}_{\Lambda}\left(\exp \lambda_{k} z\right)_{k=1}^{\infty}$, каноническим $\mathscr{E}_{\Lambda}$-рядом (к. $\mathscr{E}_{\Lambda}$-рядом), а его сумму каноническим Ё $\mathscr{E}_{\Lambda}$-решением (к. $\mathscr{E}_{\Lambda}$-решением) з.К. (1), (2).

Основное содержание остальной части данной работы составляет выяснение условий сходимости к. $\mathscr{E}_{\Lambda}$-ряда и степени гладкости к. $\mathscr{E}_{\Lambda}$-решения з.K. (1), (2).

\section{§ 4. Случай $\rho_{2} \leqslant 0$}

Так как для любой ветви функции $\mu(\lambda)$ существует конечный предел $\lim _{\lambda \rightarrow \infty} \mu(\lambda) \lambda^{-\rho_{2}}$, то в случае, когда $\rho_{2} \leqslant 0$, имеем $\nu:=\sup \left\{\left|\mu_{j}\left(\lambda_{k}\right)\right|: k \geqslant 1\right.$, $1 \leqslant j \leqslant N\}<+\infty$. Положим $\beta=\max \left\{\left(p_{l}-1\right): 1 \leqslant l \leqslant N\right\}$ и введем банахово пространство $\mathscr{E}_{2}(\nu, \beta)$ целых функций $y\left(z_{2}\right)$ таких, что

$$
\|y\|_{2}:=\sup _{z_{2} \in \mathbb{C}} \frac{\left|y\left(z_{2}\right)\right| \exp \left(-\nu\left|z_{2}\right|\right)}{\left|z_{2}\right|^{\beta}+1}<+\infty .
$$

Нетрудно проверить, что оператор дифференцирования определен и непрерывен в $\mathscr{E}_{2}(\nu, \beta)$. Кроме того, $z_{2}^{n} \exp \alpha z_{2} \in \mathscr{E}_{2}(\nu, \beta)$ при любом целом $n \geqslant 0$, если $|\alpha|<\nu$, и при $n \leqslant \beta$, если $|\alpha|=\nu$. Пусть $\mathscr{E}_{1}\left(\mathscr{F}_{1}\right)$ - ПОЛВП функций, обладаюших указанными выше (в $\S 1,3)$ свойствами, и пусть $\mathscr{P}=\{p\}$ - определяющий топологию в $\mathscr{E}_{1}\left(\mathscr{F}_{1}\right)$ набор преднорм. Введем пространство $\left\{\mathscr{E}_{1}\left(\mathscr{F}_{1}\right), \mathscr{E}_{2}(\nu, \beta)\right\}$ отображений $f\left(z_{1}, z_{2}\right): \mathscr{F}_{1} \times \mathbb{C} \rightarrow \mathbb{C}$ таких, что:

1) $\forall z_{1} \in \mathscr{F}_{1} \quad f\left(z_{1}, z_{2}\right) \in \mathscr{E}_{2}(\nu ; \beta)$;

2) $\forall z_{2} \in \mathbb{C} \quad f\left(z_{1}, z_{2}\right) \in \mathscr{E}_{1}\left(\mathscr{F}_{1}\right)$;

3) $\forall p \in \mathscr{P} \quad(p) f\left(z_{1}, z_{2}\right):=\sup _{z_{2} \in \mathbb{C}} \frac{p\left(f\left(z_{1}, z_{2}\right)\right) \exp \left(-\nu\left|z_{2}\right|\right)}{\left|z_{2}\right|^{\beta}+1}<\infty$.

Ясно, что $\left\{\mathscr{E}_{1}\left(\mathscr{F}_{1}\right), \mathscr{E}_{2}(\nu, \beta)\right\} \subset\left(\mathscr{E}_{1}\left(\mathscr{F}_{1}\right) ; \mathscr{E}_{2}(\nu, \beta)\right)$.

Если определить топологию в $\left\{\mathscr{E}_{1}\left(\mathscr{F}_{1}\right), \mathscr{E}_{2}(\nu, \beta)\right\}$ набором преднорм $(\mathscr{P})=$ $\{(p)\}_{p \in \mathscr{P}}$, то можно убедиться в том, что $\left\{\mathscr{E}_{1}\left(\mathscr{F}_{1}\right), \mathscr{E}_{2}(\nu, \beta)\right\}-$ ПОЛВП. 
TeOPEMA 1. Пусть $\rho_{2} \leqslant 0, \quad \mathscr{F}_{1} \subset \mathbb{C}, \mathscr{E}_{1}\left(\mathscr{F}_{1}\right)-$ ПОЛВП такое, ито $\mathscr{E}_{1}\left(\mathscr{F}_{1}\right) \hookrightarrow C^{\infty}\left(\mathscr{F}_{1}\right), \mathscr{E}_{1}\left(\mathscr{F}_{1}\right)$ инвариантно относительно дифференцирования и $\mathscr{E}_{1}\left(\mathscr{F}_{1}\right)$ обладает АПС $\mathscr{E}_{\Lambda}=\left(\exp \lambda_{k} z_{1}\right)_{k=1}^{\infty}$, где $\lim _{k} \lambda_{k}=\infty$. Пусть еще $\forall \alpha \in \mathbb{C} y^{\prime}-\alpha y-$ эпиморфизм $\mathscr{E}_{1}\left(\mathscr{F}_{1}\right)$.

Тогда для любых начальных функций $\varphi_{0}, \varphi_{1}, \ldots, \varphi_{m-1}$ из $\mathscr{E}_{1}\left(\mathscr{F}_{1}\right)$ существует к. $\mathscr{E}_{\Lambda}$-решение (5) з.К. (1), (2) из $\left\{\mathscr{E}_{1}\left(\mathscr{F}_{1}\right), \mathscr{E}_{2}(\nu, \beta)\right\}$. Коэффициенты $a_{j, s}^{(k)}$ ряда (5) определяются из системы (7) по коэффициентам $b_{k, n}$ разложсений начальных функций в абсолютно сходящиеся в $\mathscr{E}_{1}\left(\mathscr{F}_{1}\right)$ ряды (6) по АПС Еீ, а сам ряд (5) сходится абсолютно в $\left\{\mathscr{E}_{1}\left(\mathscr{F}_{1}\right), \mathscr{E}_{2}(\nu, \beta)\right\}$.

ДокАЗАТЕЛЬСТВо. Пусть $\mathscr{E}_{\Lambda}=\left\{\exp \lambda_{k} z_{1}\right\}_{k=1}^{\infty}-$ АПС в $\mathscr{E}_{1}\left(\mathscr{F}_{1}\right)$ такая, что $\lim _{k} \lambda_{k}=\infty$. Пусть, далее, $\varphi_{j}\left(z_{1}\right), 0 \leqslant j \leqslant m-1,-$ набор любых $m$ функций из $\mathscr{E}_{1}\left(\mathscr{F}_{1}\right)$, и пусть $(6)$ - какие-либо разложения этих функций в абсолютно сходяшиеся в $\mathscr{E}_{1}\left(\mathscr{F}_{1}\right)$ ряды по $\mathscr{E}_{\Lambda}$. Из непрерывности оператора дифференцирования в $\mathscr{E}_{1}\left(\mathscr{F}_{1}\right)$ следует, что $\forall l=0,1, \ldots, \forall p \in \mathscr{P}, \forall j \leqslant m-1$

$$
\sum_{k=1}^{\infty}\left|b_{k, j}\right|\left|\lambda_{k}\right|^{l} p\left(\exp \lambda_{k} z_{1}\right)<\infty .
$$

Далее, $\forall z_{2} \in \mathbb{C}, \forall k \geqslant 1, \forall s \leqslant p_{j}-1, \forall j \leqslant N$

$$
\left|z_{2}\right|^{s}\left|\exp \mu_{j}\left(\lambda_{k}\right) z_{2}\right| \leqslant\left(\left|z_{2}\right|^{\beta}+1\right) \exp \nu\left|z_{2}\right| .
$$

С учетом (9) имеем $\forall p \in \mathscr{P}$

$$
\begin{aligned}
& p\left(a_{j, s}^{(k)} z_{2}^{s} \exp \left[\lambda_{k} z_{1}+\mu_{j}\left(\lambda_{k}\right) z_{2}\right]\right)=\left|a_{j, s}^{(k)}\right|\left|z_{2}\right|^{s}\left|\exp \mu_{j}\left(\lambda_{k}\right) z_{2}\right| p\left(\exp \lambda_{k} z_{1}\right) \\
& \leqslant\left(\left|z_{2}\right|^{\beta}+1\right)\left(\exp \nu\left|z_{2}\right|\right) p\left(\exp \lambda_{k} z_{1}\right) \cdot D\left|\lambda_{k}\right|^{T} \sum_{k=0}^{m-1}\left|b_{k, n}\right| .
\end{aligned}
$$

Отсюда видно, что ряд (5) при любом фиксированном $z_{2} \in \mathbb{C}$ сходится абсолютно в $\mathscr{E}_{1}\left(\mathscr{F}_{1}\right)$, и потому $w_{0}\left(z_{1}, z_{2}\right) \in \mathscr{E}_{1}\left(\mathscr{F}_{1}\right)$. Более того, из приведенных оценок следует, что ряд (5) сходится абсолютно в $\left\{\mathscr{E}_{1}\left(\mathscr{F}_{1}\right), \mathscr{E}_{2}(\nu, \beta)\right\}$. Так как топология в $\mathscr{E}_{1}\left(\mathscr{F}_{1}\right)$ мажорирует топологию поточечной сходимости на $\mathscr{F}_{1}$, то при любом фиксированном $z_{1} \in \mathscr{F}_{1}$ ряд (5) сходится абсолютно в $\mathscr{E}_{2}(\nu, \beta)$, и потому $w_{0}\left(z_{1}, z_{2}\right) \in \mathscr{E}_{2}(\nu, \beta)$.

Заметим еше, что $z_{2}^{s} \exp \mu_{j}\left(\lambda_{k}\right) z_{2} \in \mathscr{E}_{2}\left(\mathscr{F}_{2}\right)=\mathscr{E}_{2}(\nu, \beta)$, если $s \leqslant p_{l}-1$, $l=1,2, \ldots, N$. Итак, все предположения, при которых в $\S 3$ было построено решение з.К. (1), (2), имеют место, и доказательство закончено.

ЗАмечАниЕ 1. Допустим, что з.К. (1), (2) имеет решение, представимое абсолютно сходящимся в $\left\{\mathscr{E}_{1}\left(\mathscr{F}_{1}\right), \mathscr{E}_{2}(\nu, \beta)\right\}$ рядом (5). Тогда, как показано в $\S 3$, $\forall z_{1} \in \mathscr{F}_{1}$ и при $n=0,1, \ldots, m-1$

$$
\left.\frac{\partial^{n} w\left(z_{1}, z_{2}\right)}{\partial z_{2}^{n}}\right|_{z_{2}=0}=\sum_{j=1}^{N} \sum_{s=0}^{q_{j}(n)} \sum_{k=1}^{\infty} a_{j, s}^{(k)} s ! C_{n}^{s}\left(\mu_{j, k}\right)^{n-s} \exp \lambda_{k} z_{1} .
$$

Так как $\sup \left\{\left|\mu_{j, k}\right|: 1 \leqslant j \leqslant N, k \geqslant 1\right\}<+\infty$, то из абсолютной сходимости в $\left\{\mathscr{E}_{1}\left(\mathscr{F}_{1}\right), \mathscr{E}_{2}(\nu, \beta)\right\}$ ряда $(5)$ легко получить, что ряд (10) сходится абсолютно в $\mathscr{E}_{1}\left(\mathscr{F}_{1}\right)$, и потому его сумма принадлежит $\mathscr{E}_{1}\left(\mathscr{F}_{1}\right)$. Таким образом, справедливо 
СЛЕДСТВИЕ. Пусть $\rho_{2} \leqslant 0, \mathscr{E}_{1}\left(\mathscr{F}_{1}\right)-$ то же пространство, что и в теореме 1, и пусть $\varphi_{k} \in C^{\infty}\left(\mathscr{F}_{1}\right), k=0,1, \ldots, m-1$.

Тогда равносильны такие утверждения:

1) начальные функиии $\varphi_{0}, \varphi_{1}, \ldots, \varphi_{m-1}$ принадлежат $\mathscr{E}_{1}\left(\mathscr{F}_{1}\right)$;

2) существует $к . \mathscr{E}_{\Lambda}-$ ряд, абсолютно сходящийся в $\left\{\mathscr{E}_{1}\left(\mathscr{F}_{1}\right), \mathscr{E}_{2}(\nu, \beta)\right\}$.

ЗАмечание 2 . Так как $\forall k \geqslant 1\left|\lambda_{k}\right| \geqslant R_{0}$, а число $R_{0}$ можно взять как угодно большим, то, зафиксировав сколь угодно малое $\varepsilon>0$, можно в теореме 1 в качестве $\nu$ взять число $\delta+\varepsilon$, где $\delta=0$, если $\rho_{2}<0$, и $\delta$ равно наибольшему из модулей корней уравнения

$$
0=P(x) \equiv a_{l_{m}, m} x^{m}+\sum_{\substack{0 \leqslant x \leqslant m-1 \\ l_{k}=l_{m}}} a_{l_{k}, k} x^{k}
$$

в случае, когда $\rho_{2}=0$.

Теорема 1 и ее следствие показывают, что если $\rho_{2} \leqslant 0$, то для широкого класса пространств $\mathscr{E}_{1}\left(\mathscr{F}_{1}\right)$ из принадлежности начальных функций этому пространству следует сушествование очень гладкого по $z_{2}$ решения, принадлежащего очень "узкому" пространству $\mathscr{E}_{2}(\nu, \beta)$ - подпространству пространства $[1, \infty)$ всех целых функций экспоненциального типа (ц.ф.э.т.). При этом пространство $\mathscr{E}_{2}(\nu, \beta)$ определяется параметрами $\nu$ и $\beta$, которые находятся по уравнению (1) и не зависят от выбора пространства $\mathscr{E}_{1}\left(\mathscr{F}_{1}\right)$, удовлетворяюшего указанным вьше условиям. Так как $\mathscr{E}_{1}\left(\mathscr{F}_{1}\right)$ содержит АПС экспонент $\left(\exp \lambda_{k} z\right)_{k=1}^{\infty}, \lim \lambda_{k}=\infty$, то, вообще говоpя, $[1, \infty) \subseteq \mathscr{E}_{1}\left(\mathscr{F}_{1}\right)$. Таким образом, решение "более гладко" по $z_{2}$ (принадлежит более "узкому" подпространству), чем по $z_{1}$, и степень гладкости решения з.К. (1), (2) не зависит от его гладкости по $z_{1}$. Интересно отметить, что в предельном случае, когда $l_{j}=0, j=0,1, \ldots, m$, т.е. для обыкновенного дифференциального уравнения

$$
\sum_{k=0}^{m} a_{k} \frac{d^{k} w\left(z_{2}\right)}{d z_{2}^{k}}=0, \quad w^{(j)}(0)=c_{j}, \quad j=0,1, \ldots, m-1,
$$

имеем $\rho_{2}=0$; параметр $\nu$ равен наибольшему из модулей корней уравнения $0=$ $P(x)=\sum_{k=0}^{m} a_{k} x^{k}$, a $\beta+1-$ наибольшей кратности этих корней. Нетрудно видеть, что решения 3.К. для этого уравнения с любыми начальными условиями (числами) $c_{j}$ принадлежит пространству $\mathscr{E}_{2}(\nu, \beta)$. При этом решения з.К. не могут находиться (сразу все) в пространстве $\mathscr{E}_{2}(\gamma, \delta)$, если $\gamma<\nu$ или если $\gamma=\nu$, но $\delta<\beta$.

Естественно говорить, что в случае $\rho_{2} \leqslant 0$ решение з.К. (1), (2) абсолютно гладко по $z_{2}$.

Заметим еще, что теорема 1 точна в том смысле, что пространство $\mathscr{E}_{2}(\nu, \beta)$ является "минимальным" (в шкале таких пространств) из содержаших решение 3.K. (1), (2).

Приведем примеры пространств $\mathscr{E}_{1}\left(\mathscr{F}_{1}\right)$.

1. Положим $\mathscr{F}_{1}=(-R, R), 0<R \leqslant+\infty ; \mathscr{E}_{1}\left(\mathscr{F}_{1}\right)=C^{\infty}(-R, R)$ - пространство $\Phi$ реше всех бесконечно дифференцируемых на $(-R, R)$ функций с топологией, определенной набором норм

$$
\|y\|_{n}=\max \left\{\left|y^{(k)}(x)\right|: 0 \leqslant k \leqslant n-1, x \in\left[-R_{n}, R_{n}\right]\right\}, \quad n=1,2, \ldots,
$$


где $0<R_{n} \uparrow R$. Здесь $\Lambda=\mathbb{C}$, так как $\exp \alpha z \in C^{\infty}(-R, R) \forall \alpha \in \mathbb{C}$. Из результатов работ [7], [5], [8] следует, что в $C^{\infty}(-R, R)$ имеется АПС $\left(\exp \lambda_{k} z\right)_{k=1}^{\infty}$, где $\lim \sup \frac{\left|\lambda_{k}\right|}{k}<+\infty, \lim \inf \frac{\left|\lambda_{k}\right|}{k}>0$. Очевидно, что оператор дифференцирования $D y=y^{\prime}$ непрерывен в $C^{\infty}(-R, R)$. При этом $\forall \alpha \in \mathbb{C} y^{\prime}-\alpha y-$ эпиморфизм $C^{\infty}(-R, R)$. Поэтому теорема 1 и ее следствие применимы к $C^{\infty}(-R, R)$.

2. Пусть $\mathscr{F}_{1}=\mathscr{G}$ - выпуклая область, отличная от всей комплексной плоскости, и $\mathscr{E}_{1}\left(\mathscr{F}_{1}\right)=\mathscr{H}(\mathscr{G})-$ пространство $\Phi$ реше всех аналитических в области $\mathscr{G}$ функций с топологией равномерной сходимости на компактах $\mathscr{G}$. Как впервые показано А. Ф. Леонтьевым [9] для любой ограниченной области и позднее автором [10] для любой неограниченной выпуклой области $\mathscr{G}$, в $\mathscr{H}(\mathscr{G})$ имеется АПС $\left(\exp \lambda_{k} z\right)$ такая, что $\lim \sup \frac{\ln k}{\left|\lambda_{k}\right|}=0$ (в случае, если область $\mathscr{G}$ ограничена, выполнено более сильное условие $\left.\lim \sup \frac{n}{\left|\lambda_{n}\right|}<\infty\right)$. Оператор дифференцирования непрерывен в $\mathscr{H}(\mathscr{G})$, и применимы теорема 1 и ее следствие.

3. Пусть $\mathscr{F}_{1}=\mathbb{C}$ и $\mathscr{E}_{1}\left(\mathscr{F}_{1}\right)=[\rho, \sigma]$, где $1<\rho<\infty, 0 \leqslant \sigma<\infty$ и $[\rho, \sigma]-$ пространство всех целых функций роста не выше, чем типа $\sigma$ при порядке $\rho$, с набором норм

$$
\|y\|_{n}=\sup _{z \in \mathscr{G}}|y(z)| \exp \left[-\left(\sigma+\frac{1}{n}\right)|z|^{\rho}\right], \quad n=1,2, \ldots
$$

Как известно (см., например, $\left[11\right.$, с. 1061]), в $[\rho, \sigma]$ имеется АПС $\mathscr{E}_{\Lambda}=\left(\exp \lambda_{k} z\right)_{k=1}^{\infty}$ такая, что $\lim \sup k\left|\lambda_{k}\right|^{-\rho_{1}}<\infty$, где $\rho_{1}=\frac{\rho}{\rho-1}$. И здесь применимы теорема 1 и ее следствия.

4. Аналогично показывается, что в качестве $\mathscr{E}_{1}\left(\mathscr{F}_{1}\right)$ можно взять такие индуктивные пределы (положив $\left.\mathscr{F}_{1}=\mathbb{C}\right)$ :

$$
\begin{aligned}
& {[\rho, \sigma)=\operatorname{ind}_{n} H_{n},} \\
& \quad H_{n}=\left\{y(z) \in(\mathbb{C})\left|\|y\|_{n}=\sup \right| y(z) \mid \exp \left(-\sigma_{n}|z|^{\rho}\right)<\infty\right\},
\end{aligned}
$$

где $0<\rho<\infty, 0<\sigma \leqslant \infty, 0<\sigma_{n} \uparrow \sigma$;

$$
F_{\rho}=\operatorname{ind}_{n} H_{n}
$$

$$
H_{n}=\left\{y(z) \in \mathscr{H}(\mathbb{C})\left|\|y\|_{n}:=\sup _{z \in \mathbb{C}}\right| y(z) \mid \exp \left(-|z|^{\rho_{n}}\right)<\infty\right\},
$$

где $0<\rho<\infty, 0<\rho_{n} \uparrow \rho$;

$M=\operatorname{ind}_{n} H_{n}$,

$$
H_{n}=\left\{y(z) \in \mathscr{H}(\mathbb{C}) \mid\|y\|_{n}:=\sup _{z \in \mathbb{C}} \frac{|y(z)|}{\exp |z|^{n}}<\infty\right\}
$$

(пространство всех целых функций конечного порядка) и т.д.

5. Пусть $1<\alpha<\infty, 0 \leqslant \sigma<\infty, F$ - выпуклый компакт в $\mathbb{C}$ и $\mathscr{F}(\alpha, \sigma)$-множество всех отображений $F \rightarrow \mathbb{C}$, аналитических в области (возможно, пустой) Int $F$, бесконечно дифференцируемых на $\mathscr{F}$ и таких, что

$$
\forall n \geqslant 1|y|_{n}:=\sup \left\{\left|y^{(k)}(z)\right|(k !)^{-\alpha}\left(\sigma+\frac{1}{n}\right)^{-k}: z \in F, \quad k=0,1, \ldots\right\}<\infty .
$$


Легко убедиться, что $\mathscr{F}(\alpha, \sigma)$ - пространство Фреше, инвариантное относительно дифференцирования, причем множество $\operatorname{span}\{\exp \lambda z: \lambda \in \mathbb{C}\}$ плотно в $\mathscr{F}(\alpha, \sigma)$.

Пусть $h_{F}(\varphi):=\max \left\{\operatorname{Re} z e^{-i \varphi}: z \in F\right\}$ - опорная функция компакта $F$. Применяя традиционную технику, основанную на использовании теорем типа Пели-Винера-Шварца, можно показать, что отображение $\forall \varphi \in(\mathscr{F}(\alpha, \sigma))^{\prime} \mapsto \varphi\left(e^{\lambda z}\right)$ устанавливает топологический изоморфизм между сильным сопряженным к $\mathscr{F}(\alpha, \sigma)$ пространством и пространством $E=\operatorname{ind}_{n} E_{n}$, где $\forall n \geqslant 1$

$$
\begin{aligned}
E_{n} & =\left\{x(z) \in A(\mathbb{C}) \mid\|x\|_{n}:=\sup _{\lambda \in \mathbb{C}} \frac{|x(\lambda)|}{d_{n}(\lambda)}<\infty\right\}, \\
d_{n}(\lambda) & =\left|e^{\lambda z}\right|_{n}=\exp \left[|\lambda| h_{F}(-\arg \lambda)\right] \cdot \sup _{k \geqslant 0}(k !)^{-\alpha}\left(\sigma+\frac{1}{n}\right)^{-k}|\lambda|^{k} .
\end{aligned}
$$

Иначе говоря, сильное сопряженное пространство $(\mathscr{F}(\alpha, \sigma))_{\beta}^{\prime}$ допускает ехр-описание (см. [12, с. 562]). Такое представление пространства $(\mathscr{F}(\alpha, \sigma))_{\beta}^{\prime}$ было получено в выполненных в Ростовском университете дипломных работах Н. В. Новиковой для случая, когда Int $F \neq \varnothing$, и Е.В. Тищенко для случая, когда $F$ - замкнутый отрезок.

Легко проверить, что пространство $E$ изоморфно топологически пространству $\mathscr{E}=\operatorname{ind}_{n} \mathscr{E}_{n}$, где $\mathscr{E}_{n}-B$-пространство целых функций $x(z)$ таких, что

$$
\|\widehat{x}\|_{n}:=\sup _{\lambda \in \mathbb{C}}\left[|x(\lambda)| \exp \left(-|\lambda| h_{F}(-\arg \lambda)-\left(\sigma+\frac{1}{n}\right)^{-1}|\lambda|^{\frac{1}{\alpha}}\right)\right]<\infty .
$$

Очевидно, что множество $\mathbb{C}$ слабо достаточно для пространства $\mathscr{E}=$ ind $\mathscr{E}_{n}$ (определение слабо достаточного множества см., например, в $[12, \S 1]$ ). Согласно [13] можно построить дискретное подмножество $\Lambda=\left\{\lambda_{n}: n=1,2, \ldots\right\}$ множества $\mathbb{C}$ такое, что $\lim _{n \rightarrow \infty} \lambda_{n}=\infty$ и $\Lambda$ слабо достаточно для $\mathscr{E}$. Из теоремы $\mathrm{K}$ (см. [12, с. 563]) следует, что $\mathscr{E}_{\Lambda}:=\left(\exp \lambda_{k} z\right)_{k=1}^{\infty}-\mathrm{AПC} \mathrm{в} \mathscr{F}(\alpha, \sigma)$. В данном случае $Q_{1}=F, \mathscr{E}_{1}\left(Q_{1}\right)=\mathscr{F}(\alpha, \sigma)$ и применима теорема 1.

\section{§5. Случай, когда $0<\rho_{2}<1$}

Рассмотрение з.К. (1), (2) в этой ситуации начнем с одного модельного случая, который используется ниже, в $\S 7$. Пусть $0<R<\infty, K_{R}=\{z:|z|<$ $R\}$ и $\mathscr{E}_{\Lambda}=\left(\exp \lambda_{k} z\right)_{k=1}^{\infty}-\mathrm{A \Pi C} \mathrm{в} \mathrm{пространстве} \Phi$ реше $\mathscr{H}\left(K_{R}\right)$, для которой $\lim \sup _{k \rightarrow \infty} \frac{k}{\left|\lambda_{k}\right|}<\infty$ (как отмечалось выше, такая АПС всегда сушествует). Пусть, далее, $\varphi_{j}\left(z_{1}\right)(0 \leqslant j \leqslant m-1)$ - произвольные функции из $\mathscr{H}\left(K_{R}\right)$ и $(6)-$ какие-либо их разложения в абсолютно сходяшиеся в $\mathscr{H}\left(K_{R}\right)$ ряды по системе $\mathscr{E}_{\Lambda}$. Согласно критерию абсолютной сходимости таких рядов в $\mathscr{H}\left(K_{R}\right)$ (см., например, более обший результат работы [10, с. 333-334], в котором следует положить $h(\theta) \equiv R)$ имеем $\lim \sup _{k \rightarrow \infty}\left[\left|\lambda_{k}\right|^{-1} \ln \left|b_{k, j}\right|+R\right] \leqslant 0, j=0,1, \ldots, m-1$. Учитывая оценки (9), получаем

$$
\limsup _{k \rightarrow \infty}\left[\frac{1}{\left|\lambda_{k}\right|} \ln \left|a_{j, s}^{(k)}\right|+R\right] \leqslant 0, \quad j=1, \ldots, N, \quad s=0,1, \ldots, p_{j}-1 .
$$

Зафиксируем $j \leqslant N, s \leqslant p_{j}-1$ и $r<R$. Положим

$$
\mu_{j, k}=\mu_{j}\left(\lambda_{k}\right), \quad d_{j, s}^{(k)}=a_{j, s}^{(k)} \exp \left|\lambda_{k}\right| r, \quad k=1,2, \ldots
$$


Пусть сначала $\tau_{j} \neq 0$, где, как и вьше, $\tau_{j}=\lim _{\lambda \rightarrow \infty} \lambda^{-\rho_{2}} \mu_{j}(\lambda)$. Тогда если $|z| \leqslant r$, то

$$
\limsup _{k \rightarrow \infty}\left|\mu_{j, k}\right|^{-1 / \rho_{2}} \ln \left|d_{j, s}^{(k)}\right|+\left|\tau_{j}\right|^{-1 / \rho_{2}}(R-r) \leqslant 0 .
$$

По лемме работы $\left[14\right.$, с. 509] ряд $\sum_{k=1}^{\infty} d_{j, s}^{(k)} \exp \mu_{j}\left(\lambda_{k}\right) z_{2}$ сходится абсолютно в пространстве $\left[\frac{1}{1-\rho_{2}}, \sigma_{j}\right]$, где

$$
\sigma_{j}=\left(1-\rho_{2}\right) \rho_{2}^{\frac{\rho_{2}}{1-\rho_{2}}}\left|\tau_{j}\right|^{\frac{1}{1-\rho_{2}}}(R-r)^{\frac{\rho_{2}}{\rho_{2}-1}} .
$$

Если же $\tau_{j}=0$, то из $(9),(11)$ в этом случае получаем

$$
\lim _{k \rightarrow \infty}\left|\mu_{j, k}\right|^{-1 / \rho_{2}} \ln \left|d_{j, s}^{(k)}\right|=-\infty
$$

и соответствуюший ряд $\sum_{k=1}^{\infty} d_{j, s}^{(k)} \exp \mu_{j}\left(\lambda_{k}\right) z_{2}$ сходится абсолютно в $\left[\frac{1}{1-\rho_{2}}, 0\right]$. Положим $\sigma(r)=\max \left\{\sigma_{j}: 1 \leqslant j \leqslant N\right\}$, где $\sigma_{j}$ определяются по формулам (12) в случае $\tau_{j} \neq 0$, и $\sigma_{j}=0$, если $\tau_{j}=0$. Мы получили, что при любом $r<R$ и при любом фиксированном $z_{1}$ из $\overline{K_{r}}$ ряд (5) сходится абсолютно в пространстве $\left[\frac{1}{1-\rho_{2}}, \sigma(r)\right]$, и, следовательно, его сумма $w_{0}\left(z_{1}, z_{2}\right)$ принадлежит (как функция от $\left.z_{2}\right)$ этому пространству.

Пусть теперь $z_{2}$ - любое фиксированное число, и положим $c_{j, s}^{(k)}=a_{j, s}^{(k)} \exp \left|\mu_{j, k}\right| \times$ $\left|z_{2}\right|$. Тогда из (11) следует, что $\lim \sup _{k \rightarrow \infty}\left[\left|\lambda_{k}\right|^{-1} \ln \left|c_{j, s}^{(k)}\right|+R\right] \leqslant 0$ и ряд (5) сходится абсолютно в $\mathscr{H}\left(K_{R}\right)$. Поэтому $w_{0}\left(z_{1}, z_{2}\right) \in \mathscr{H}\left(K_{R}\right) \forall z_{2} \in \mathbb{C}$. По теореме Гартогса $w_{0}\left(z_{1}, z_{2}\right) \in \mathscr{H}\left(K_{R} \times \mathbb{C}\right)$.

Введем пространство $\mathscr{E}\left(\rho_{2}, R\right)$ функций $y\left(z_{1}, z_{2}\right)$ из $\mathscr{H}\left(K_{R} \times \mathbb{C}\right)$ таких, что $\forall r \in$ $[0, R), \forall n \geqslant 1$

$$
|y|_{r, n}:=\sup _{\left|z_{1}\right| \leqslant r, z_{2} \in \mathbb{C}}\left|y\left(z_{1}, z_{2}\right)\right| \exp \left[-\left(\sigma(r)+\frac{1}{n}\right)\left|z_{2}\right|^{\frac{1}{1-\rho_{2}}}\right]<\infty .
$$

Нетрудно убедиться, что $\mathscr{E}\left(\rho_{2}, R\right)$ - пространство $\Phi$ реше со счетным набором норм $\|y\|_{n}:=|y|_{R\left(1-\frac{1}{n}\right), n}, \quad n=2,3, \ldots$ Из приведенных вьше выкладок легко вывести, что если $\varphi_{j}(j=0,1, \ldots, m-1)$ - произвольные функции из $\mathscr{H}\left(K_{R}\right)$ и $(11)-$ какие-либо абсолютно сходящиеся в $\mathscr{H}\left(K_{R}\right)$ их разложения по АПС $\mathscr{E}_{\Lambda}$, то построенньй по коэффициентам этих разложений ряд (5) сходится абсолютно в $\mathscr{E}\left(\rho_{2}, R\right)$. Его сумма принадлежит $\mathscr{E}\left(\rho_{2}, R\right)$ и подавно пространству $\left(\mathscr{H}\left(K_{R}\right) ;\left[\frac{1}{1-\rho_{2}}, \infty\right)\right)$. Обратно, пусть начальные функции $\varphi_{j}(j=0,1, \ldots, m-1)$ аналитичны в окрестности начала координат, и пусть существует решение з.К. (1), (2) из $\mathscr{H}\left(K_{R} \times \mathbb{C}\right)$. Тогда $\forall j \geqslant 0 \frac{\partial^{j} w\left(z_{1}, z_{2}\right)}{\partial z_{2}^{j}} \in \mathscr{H}\left(K_{R} \times \mathbb{C}\right)$, и потому $\left.\frac{\partial^{j} w\left(z_{1}, z_{2}\right)}{\partial z_{2}^{j}}\right|_{z_{2}=0} \in \mathscr{H}\left(K_{R}\right)$. Сформулируем полученный результат.

TEOPEMA 2. Пусть $0<\rho_{2}<1,0<R<\infty$, и пусть функиии $\varphi_{j}\left(z_{1}\right)$ аналитичны в окрестности начала координат $(0 \leqslant j \leqslant m-1)$.

Тогда следующие утверждения равносильны: 
1) $\varphi_{j} \in \mathscr{H}\left(K_{R}\right), j=0,1, \ldots, m-1$;

2) существует решение з.К. (1), (2) из $\mathscr{H}\left(K_{R} \times \mathbb{C}\right)$;

3) существует решение з.К. (1), (2) из $\mathscr{E}\left(\rho_{2}, R\right)$;

4) существует к. $\varepsilon_{\Lambda}$-ряд, абсолютно сходящийся в $\mathscr{E}\left(\rho_{2}, R\right)$.

Заметим, что из абсолютной сходимости ряда $(5)$ в $\mathscr{E}\left(\rho_{2}, R\right)$ следует, что при любом фиксированном $z_{2} \in \mathbb{C}$ он сходится абсолютно в $\mathscr{H}\left(K_{R}\right)$, а при любом фиксированном $z_{1} \in K_{R}-$ в $\left[\frac{1}{1-\rho_{2}}, \sigma\left(\left|z_{1}\right|\right)\right]$.

Тем же методом (но более простыми выкладками) доказывается

Tеорема 3. Пусть $0<\rho_{2}<1$, и пусть начальные функции $\varphi_{j}\left(z_{1}\right)(0 \leqslant j \leqslant$ $m-1)$ аналитичны в окрестности начала координат.

Тогда следующие утверждения равносильны:

1) $\varphi_{j} \in \mathscr{H}(\mathbb{C}), \quad j=0,1, \ldots, m-1$;

2) существует решение з.К. (1), (2) из $\mathscr{H}\left(\mathbb{C}^{2}\right)$;

3) существует решение з.К. (1), (2) из $\left(\mathscr{H}(\mathbb{C}) ;\left[\frac{1}{1-\rho_{2}}, 0\right]\right)$;

4) существует решение з.К. (1), (2), представимое в виде к. $\mathscr{E}_{\Lambda}$-ряда (5), абсолютно сходящегося в пространстве фреше $\mathscr{E}\left(\rho_{2}, \infty\right)$ всех функиий $y\left(z_{1}, z_{2}\right)$ uз $\mathscr{H}\left(\mathbb{C}^{2}\right)$ maких, что $\forall n \geqslant 1$

$$
\left\|y\left(z_{1}, z_{2}\right)\right\|_{n}:=\sup \left\{\left|y\left(z_{1}, z_{2}\right)\right| \exp \left(-\frac{\left|z_{2}\right|^{\frac{1}{1-\rho_{2}}}}{n}\right):\left|z_{1}\right| \leqslant n, z_{2} \in \mathbb{C}\right\}<\infty .
$$

Пусть, далее, $\delta>1,0 \leqslant \sigma<\infty, \tau=\max \left\{\tau_{j}: 1 \leqslant j \leqslant m\right\}$. Полагая $\mathscr{F}_{1}=\mathscr{F}_{2}=\mathbb{C}, \mathscr{E}_{1}\left(\mathscr{F}_{1}\right)=[\delta, \sigma], \mathscr{E}_{2}\left(\mathscr{F}_{2}\right)=\left[\delta_{0}, \sigma_{0}\right]$, где $\delta_{0}\left(\delta-\delta \rho_{2}+\rho_{2}\right)=\delta$, $\left(\delta_{0} \sigma_{0}\right)^{1 / \delta_{0}} \cdot(\delta \sigma)^{-\rho_{2} / \delta}=\tau\left(\rho_{2}\right)^{\frac{\rho_{2}(\delta-1)}{\delta}}$, и используя те же рассуждения, что и при доказательстве теоремы 2 , приходим к такому результату.

Teopema 4. Пусть $0<\rho_{2}<1, \quad \delta>1, \quad 0 \leqslant \sigma<\infty, \quad \varphi_{j}\left(z_{1}\right) \in \mathscr{H}(\mathbb{C})$, $j=0,1, \ldots, m-1$. Тогда следующие утверждения равносильны:

1) $\varphi_{j} \in[\delta, \sigma], \quad j=0,1, \ldots, m-1$;

2) существует решение з.К. (1), (2) из $([\delta, \sigma] ; \mathscr{H}(\mathbb{C}))$;

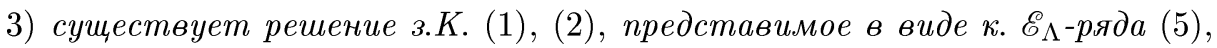
абсолютно сходящегося в пространстве $\mathscr{E}\left(\delta, \sigma ; \delta_{0}, \sigma_{0}\right)$ всех целых функиий $y\left(z_{1}, z_{2}\right)$ uз $\mathscr{H}\left(\mathbb{C}^{2}\right)$ maкux, umo $\forall n \geqslant 1$

$$
\left\|y\left(z_{1}, z_{2}\right)\right\|_{n}:=\sup _{z_{1}, z_{2} \in \mathbb{C}}\left|y\left(z_{1}, z_{2}\right)\right| \exp \left[-\left(\sigma+\frac{1}{n}\right)\left|z_{1}\right|^{\delta}-\left(\sigma_{0}+\frac{1}{n}\right)\left|z_{2}\right|^{\delta_{0}}\right]<\infty .
$$

Наконец, для введенного в $§ 4$ (пример 5) пространства $\mathscr{F}(\alpha, \sigma)$ ультрадифференцируемых на выпуклых компактах $F$ в $\mathbb{C}$ функций аналогичными рассуждениями получается такой результат.

TeOpemA 5. Пусть $0<\rho_{2}<1,0 \leqslant \sigma<\infty, 0 \leqslant \alpha<\frac{1}{\rho_{2}}$, u nусть $F-$ произвольный выпукльй компакт в $\mathbb{C}$. Тогда для любых начальных функчий $\varphi_{n}\left(z_{1}\right)(n=0,1, \ldots, m-1)$ из $\mathscr{F}(\alpha, \sigma)$ существует решение з.К. (1), (2) из 
$\left(\mathscr{F}(\alpha, \sigma) ;\left[\frac{1}{1-\alpha \rho_{2}}, \sigma_{0}\right]\right)$, где $\sigma_{0}=\left(1-\alpha \rho_{2}\right)\left(\sigma \rho_{2}\right)^{\frac{\alpha \rho_{2}}{1-\alpha \rho_{2}}}$. Это решение представляется в виде к. ஜ́ $\mathscr{\complement}_{\Lambda}$-ряда $(5)$, абсолютно сходящегося в $\left\{\mathscr{F}(\alpha, \sigma) ;\left[\frac{1}{1-\alpha \rho_{2}}, \sigma_{0}\right]\right\}$.

В $[4, \S 5]$ получен также аналогичный (но более сложно формулируемый) результат, относящийся к случаю $\alpha=\frac{1}{\rho_{2}}, F=\{z:|z| \leqslant R\}, 0<R<\infty$.

Из теорем 2-6 видно, что в случае $0<\rho_{2}<1$ характер гладкости решения з.К. (1), (2) по переменной $z_{2}$ зависит, вообще говоря, от пространства $\mathscr{E}_{1}\left(\mathscr{F}_{1}\right), \mathrm{k}$ которому должны принадлежать начальные функции $\varphi_{j}$, но каждый раз гладкость в случае аналитических или ультрадифференцируемых начальных данных по $z_{2}$ выше, чем по $z_{1}$ (в том смысле, что $\mathscr{E}_{2} \hookrightarrow \mathscr{E}_{1}$ ). Поэтому естественно говорить при $\rho_{2} \in(0,1)$, что в этом случае имеет место усиленная ("улучшенная") гладкость по $z_{2}$ решения з.K.

\section{§ 6. Случай $\rho_{2}=1$}

Рассмотрение этой ситуации начнем со случая, когда начальные функции $\varphi_{j}$ аналитичны в некоторой области. Пусть $\mathscr{G}_{0}-$ вьпуклая (не обязательно ограниченная), содержашая начало координат область в $\mathbb{C}$ с опорной функцией $g(-\varphi)$; $\left\{g_{n}(\varphi)\right\}_{n=1}^{\infty}-$ последовательность $2 \pi$-периодических ограниченных тригонометрически выпуклых (см. [15, с. 75]) функций со следующими свойствами:

1) $\forall n \geqslant 1, \forall \theta \in[0,2 \pi] g(\theta)>g_{n}(\theta) ; \min _{\theta}\left(g_{n+1}(\theta)-g_{n}(\theta)\right)>0$;

2) если $h(\theta)$ - любая $2 \pi$-периодическая тригонометрически выпуклая функция, для которой $h(\theta)<g(\theta) \forall \theta \in[0,2 \pi]$, то $\exists N \geqslant 1: \forall n \geqslant N, \forall \theta \quad g_{n}(\theta)>h(\theta)$.

Как известно [16], такая последовательность всегда найдется. Если $\mathscr{G}_{n}-$ выпуклое замкнутое множество с опорной функцией $g_{n}(-\theta)$, то $\mathscr{G}_{n}-$ компакт области $\mathscr{G}_{0}$, и топологию Фреше в $\mathscr{H}\left(\mathscr{G}_{0}\right)$ можно задать набором преднорм $|y|_{n}:=\max \{|y(z)|:$ $\left.z \in \mathscr{G}_{n}\right\}$. Всегда можно считать, что $0 \in \mathscr{G}_{n}, n=1,2, \ldots$

Для любой точки $\tau \in \mathbb{C}$ положим

$$
\left(\mathscr{G}_{0}\right)_{\tau}=\left\{\left(z_{1}, z_{2}\right) \in \mathbb{C}^{2}: z_{1}+\tau z_{2} \in \mathscr{G}_{0}\right\} .
$$

Пусть $\tau_{1}, \tau_{2}, \ldots, \tau_{m}$ - все корни многочлена $P(x)=\sum_{k \in N_{2}} a_{l_{k}, k} x^{k}$ (они не обязательно различны). Положим $\mathscr{P} \mathscr{G}_{0}=\bigcap_{k=1}^{m}\left(\mathscr{G}_{0}\right)_{\tau_{k}}$. Очевидно, что $\mathscr{P} \mathscr{G}_{0}-$ выпуклая область в $\mathbb{C}^{2}$, содержашая множество $\mathscr{G}_{0} \times\{0\}$.

Tеорема 6. Если $\varphi_{k}, \quad k=0,1, \ldots m-1,-$ любье функции из $\mathscr{H}\left(\mathscr{G}_{0}\right)$, mо решение з.К. (1), (2) в $\mathscr{H}\left(\mathscr{P} \mathscr{G}_{0}\right)$ существует. При этом если $\mathscr{E}_{\Lambda}=\left(\exp \lambda_{k} z\right)_{k=1}^{\infty}-$ любая АПС в $\mathscr{H}\left(\mathscr{G}_{0}\right)$, для которой $\lim _{k \rightarrow \infty} \frac{\ln k}{\lambda_{k}}=0$, а (6) - какие-либо разложения начальных функиий $\varphi_{k}$ в абсолютно сходящиеся в $\mathscr{H}\left(\mathscr{G}_{0}\right)$ ряды по системе $\mathscr{E}_{\Lambda}$, то в качестве решения з.К. (1), (2)можно взять сумму $w_{0}\left(z_{1}, z_{2}\right)$ к. $\mathscr{E}_{\Lambda}$-ряда (5), который сходится абсолютно в пространстве $\mathscr{H}\left(\mathscr{P} \mathscr{G}_{0}\right)$.

ДокАЗАТЕЛЬСТво. Теорема будет доказана, если мы покажем, что при любых $j$ и $s$ таких, что $1 \leqslant j \leqslant N, 0 \leqslant s_{j} \leqslant p_{j}-1$, ряд

$$
\sum_{k=1}^{\infty} a_{j, s}^{(k)}\left(z_{2}\right)^{s} \exp \left(\lambda_{k} z_{1}+\mu_{j}\left(\lambda_{k}\right) z_{2}\right)
$$


сходится абсолютно в $\mathscr{H}\left(\mathscr{P} \mathscr{G}_{0}\right)$.

Пусть $F$ - любой компакт области $\mathscr{P} \mathscr{G}_{0}$. Тогда множество $F_{1}=\bigcup_{j=1}^{m}\left\{z_{1}+\tau_{j} z_{2}\right.$ : $\left.\left(z_{1}, z_{2}\right) \in F\right\}$ будет компактом в $\mathscr{G}_{0}$. Следовательно, $\exists K: F_{1} \subset \mathscr{G}_{K}$. Положим $M=\sup \left\{\left|z_{2}\right|:\left(z_{1}, z_{2}\right) \in F\right\}$. Тогда $M<\infty$ и при $k=1,2, \ldots$

$$
\begin{aligned}
& \max \left\{\operatorname{Re} \lambda_{k}\left(z_{1}+\tau_{j} z_{2}\right):\left(z_{1}, z_{2}\right) \in F\right\} \leqslant \max \left\{\operatorname{Re} \lambda_{k} v: v \in \mathscr{G}_{K}\right\} \\
& \max \left\{\operatorname{Re}\left(\lambda_{k} z_{1}+\mu_{j}\left(\lambda_{k}\right) z_{2}\right):\left(z_{1}, z_{2}\right) \in F\right\} \\
& \leqslant \max \left\{\operatorname{Re}\left(\lambda_{k}\left(z_{1}+\tau_{j} z_{2}\right):\left(z_{1}, z_{2}\right) \in F\right\}\right. \\
& \quad+\max \left\{\operatorname{Re} \lambda_{k} \varepsilon_{k} z_{2}:\left(z_{1}, z_{2}\right) \in F\right\} \\
& \leqslant \max \left\{\operatorname{Re} \lambda_{k} v: v \in \mathscr{G}_{K}\right\}+\operatorname{Re}\left|\lambda_{k}\right| \varepsilon_{k} M
\end{aligned}
$$

где $\lim _{k \rightarrow \infty} \varepsilon_{k}=0$.

Далее, если $s \leqslant \sum_{j=1}^{N} p_{j}$, то $\max \left\{\left|z_{2}\right|^{s}:\left(z_{1}, z_{2}\right) \in F\right\} \leqslant(M+1)^{m}$. Отсюда с учетом (9) получаем для любого $k \geqslant 1$

$$
\begin{aligned}
\gamma_{j, s}^{(k)}:= & \left|a_{j, s}^{(k)}\right| \max \left\{\left|z_{2}\right|^{s} \exp \operatorname{Re}\left(\lambda_{k} z_{1}+\mu_{j}\left(\lambda_{k}\right) z_{2}\right):\left(z_{1}, z_{2}\right) \in F\right\} \\
\leqslant & (M+1)^{m} \exp \left\{\max \left(\operatorname{Re} \lambda_{k} v: v \in \mathscr{G}_{K}\right)+\operatorname{Re} M\left|\lambda_{k}\right| \varepsilon_{k}\right\} \\
& \times D \sum_{n=0}^{m-1}\left|b_{k, n}\right| \cdot\left|\lambda_{k}\right|^{T}
\end{aligned}
$$

Если $k \geqslant k_{0}$, то

$$
\begin{aligned}
\gamma_{j, s}^{(k)} & \leqslant D_{1} \sum_{n=0}^{m-1}\left|b_{k, n}\right| \exp \left|\lambda_{k}\right| h_{K+1}\left(\arg \lambda_{k}\right) \\
& =D_{1} \sum_{n=0}^{m-1}\left|b_{k, n}\right| \exp \left(\max \left\{\operatorname{Re} \lambda_{k} v: v \in \mathscr{G}_{K+1}\right\}\right) .
\end{aligned}
$$

Учитывая, что все ряды (6) сходятся абсолютно в $\mathscr{H}\left(\mathscr{G}_{0}\right)$, находим из $(14)$, что ряд (5) сходится абсолютно в $\mathscr{H}\left(\mathscr{P} \mathscr{G}_{0}\right)$.

Выясним степень точности теоремы 6. Обозначим символом $(H)(0)$ сечение плоскостью $z_{2}=0$ любой вьпуклой области $H \subset \mathbb{C}^{2}$. Область $\mathscr{P} \mathscr{G}_{0}$, в которой согласно теореме 6 гарантируется существование голоморфного решения з.К. (1), $(2)$, обладает тем свойством, что $\left(\mathscr{P} \mathscr{G}_{0}\right)(0)=\mathscr{G}_{0}$. Обозначим символом $B$ множество всех выпуклых областей $D \subset \mathbb{C}^{2}$ таких, что $(D)(0) \supseteq \mathscr{G}_{0}$ и з.К. (1), (2) разрешима в $\mathscr{H}(D)$ для любых функций $\varphi_{j}$ из $\mathscr{H}\left(\mathscr{G}_{0}\right), j=0,1, \ldots, m-1$. Если в качестве $\varphi_{0}$ взять функцию, полная область существования которой совпадает с $\mathscr{G}_{0}$, то получим, что соответствуюшее решение з.K. (1), (2), в случае его существования, не может быть аналитическим в любой выпуклой области $T \subset \mathbb{C}^{2}$ такой, что $(T)(0) \supseteq \mathscr{G}_{0}$ и $(T)(0)$ содержит хотя бы одну граничную точку $\mathscr{G}_{0}$. Таким образом, если $T \in B$, то $(T)(0)=\mathscr{G}_{0}$, и в теореме 6 область $\mathscr{P} \mathscr{G}_{0}$ нельзя заменить выпуклой областью $T \subset \mathbb{C}^{2}$ такой, что $(T)(0) \supseteq \mathscr{G}_{0}$ и $(T)(0) \cap \partial \mathscr{G}_{0} \neq \varnothing$. 
Заметим еще, что если $\mathscr{G}_{0}-$ любая выпуклая область в $\mathbb{C}, \mathscr{G}=\mathscr{P} \mathscr{G}_{0}$ и если начальные функции $\varphi_{k}$ аналитичны в окрестности начала координат, то из сушествования голоморфного в $\mathscr{P} \mathscr{G}_{0}$ решения $w\left(z_{1}, z_{2}\right)$ з.К. $(1),(2)$ следует, что при $k=0,1, \ldots, m-1$

$$
\varphi_{k}\left(z_{1}\right)=\left.\frac{\partial^{k} w\left(z_{1}, z_{2}\right)}{\partial z_{2}^{k}}\right|_{z_{2}=0} \in \mathscr{H}\left(\mathscr{G}_{0}\right) .
$$

Таким образом, справедлива

ТЕОРЕМА 7. Пусть $\rho_{2}=1$ и начальные функции $\varphi_{j}\left(z_{1}\right)$ аналитичны в окрестности начала координат. Тогда следующие утверждения равносильHbl:

а) существует решение з.К. (1), (2) из $\mathscr{H}\left(\mathscr{P} \mathscr{G}_{0}\right)$;

б) начальные функции $\varphi_{j}$ допускают аналитическое продолжение во всю область $\mathscr{G}_{0}$;

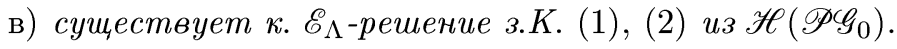

Как и в рассмотренных случаях $\rho_{2} \leqslant 0,0<\rho_{2}<1$, метод данной работы позволяет и в случае $\rho_{2}=1$ получить решение з.К. (1), (2) при различных пространствах $\mathscr{E}_{1}\left(\mathscr{F}_{1}\right)$ начальных заданий, обладающих указанными в $\S 1-3$ свойствами. Приведем примеры таких пространств.

Положим $\mathscr{F}_{1}=\mathbb{C}, \mathscr{E}_{1}\left(\mathscr{F}_{1}\right)=[\delta, \sigma]$, где $1<\delta<\infty, 0 \leqslant \sigma<\infty$. Пусть $\mathscr{E}_{\Lambda}:=\left\{\exp \lambda_{k} z_{1}\right\}_{k=1}^{\infty}-$ какая-либо АПС в $[\delta, \sigma]$ такая, что $\lim \sup _{n} n\left|\lambda_{n}\right|^{-\delta}<\infty$.

Положим $\tau_{0}=\max \left\{\left|\tau_{j}\right|^{\delta}: j=1, \ldots, m\right\}$ и введем пространство Фреше $\mathscr{E}\left[\delta, \sigma ; \tau_{0}\right]$ всех целых функций $y\left(z_{1}, z_{2}\right)$ таких, что $\forall n \geqslant 1$

$$
\|y\|_{n}:=\sup _{\left(z_{1}, z_{2}\right) \in \mathbb{C}^{2}} \frac{\left|y\left(z_{1}, z_{2}\right)\right|}{\exp \left[\left(\sigma+\frac{1}{n}\right)\left|z_{1}\right|^{\delta}+\left(\sigma \tau_{0}+\frac{1}{n}\right)\left|z_{2}\right|^{\delta}\right]}<\infty .
$$

Стандартными для данной работы рассуждениями доказывается

Tеорема 8. Пусть $\rho_{2}=1, \quad 1<\delta<\infty, \quad 0 \leqslant \sigma<\infty, \varphi_{j} \in \mathscr{H}(\mathbb{C})$, $j=0, \ldots, m-1$. Тогда следующие утверәсдения равносильны:

1) $\varphi_{j} \in[\delta, \sigma], j=0, \ldots, m-1$;

2) существует решение з.К. (1), (2) из $\mathscr{E}\left[\delta, \sigma ; \tau_{0}\right]$;

3) существует $\kappa . \mathscr{E}_{\Lambda}-$ яяд (5), абсолютно сходящийся в $\mathscr{E}\left[\delta, \sigma ; \tau_{0}\right]$.

Пусть теперь $1<\delta<\infty, 0<\sigma \leqslant \infty$ и $[\delta, \sigma)=\operatorname{ind}\left[\delta, \sigma_{n}\right], 0<\sigma_{n} \uparrow \sigma$.

Обозначим символом $\mathscr{E}\left(\delta, \sigma ; \tau_{0}\right)$ пространство всех целых функций $y\left(z_{1}, z_{2}\right)$ таких, что $\forall z_{1} \in \mathbb{C} y\left(z_{1}, z_{2}\right) \in\left[\delta, \sigma \tau_{0}\right)$ и $\forall z_{2} \in \mathbb{C} y\left(z_{1}, z_{2}\right) \in[\delta, \sigma)$. Рассуждениями, аналогичными вышеизложенным, нетрудно показать, что если $\rho_{2}=1$, то следующие утверждения равносильны:

1) $\varphi_{j} \in[\delta, \sigma), j=0, \ldots, m-1$;

2) сушествует решение з.К. (1), (2) из $\mathscr{E}\left(\delta, \sigma ; \tau_{0}\right)$.

В качестве последнего примера рассмотрим уже знакомое пространство $C^{\infty}(-R, R)$ всех бесконечно дифференцируемых на интервале $(-R, R)$ функций, где $0<R<\infty$, и топология задается набором преднорм

$$
\|y\|_{n}:=\max \left\{\left|y^{(k)}(x)\right|: 0 \leqslant k \leqslant n, x \in\left[-R_{n}, R_{n}\right]\right\}
$$


$0<R_{n} \uparrow R, n=1,2, \ldots$ Очевидно, что $\forall \lambda \in \mathbb{C}, \forall n \geqslant 1$

$$
\|\exp \lambda x\|_{n}=\max \left\{|\lambda|^{n}, 1\right\} \exp |\lambda| R_{n}|\cos \arg \lambda| .
$$

Согласно [7] в $C^{\infty}(-R, R)$ имеется АПС $\mathscr{E}_{\Lambda}$ экспонент вида

$$
\left\{\exp \frac{i k \pi x}{R}, \exp m x: k=0, \pm 1, \pm 2, \ldots ; m= \pm 1, \pm 2, \ldots\right\} .
$$

При этом каждый из рядов по системе $\mathscr{E}_{\Lambda}$

$$
\sum_{k=-\infty}^{\infty} c_{k} \exp \frac{i k \pi x}{R}+\sum_{m=1}^{\infty} d_{m} \exp m x+\sum_{m=1}^{\infty} d_{-m} \exp (-m x)
$$

сходится абсолютно в $C^{\infty}(-R, R)$ тогда и только тогда, когда $\forall n \geqslant 1$

$$
\sum_{k=-\infty}^{\infty}|k|^{n}\left|c_{k}\right|+\sum_{m=1}^{\infty}\left|d_{m}\right| \exp m R_{n}+\sum_{m=1}^{\infty}\left|d_{-m}\right| \exp m R_{n}<\infty
$$

Положим $\lambda_{0,1}=0, \lambda_{2 k, 1}=-\frac{k \pi i}{R}, \lambda_{2 k-1,1}=\frac{k \pi i}{R}, \lambda_{2 k, 2}=-k, \lambda_{2 k-1,2}=k$, $k=1,2, \ldots$ Тогда начальные функции из $C^{\infty}(-R, R)$ можно представить в виде абсолютно сходяшихся рядов

$$
\varphi_{j}\left(x_{1}\right)=\sum_{k=0}^{\infty} C_{k, j} \exp \lambda_{k, 1} x_{1}+\sum_{k=1}^{\infty} D_{k, j} \exp \lambda_{k, 2} x_{1},
$$

где $j=0, \ldots, m-1, x_{1} \in(-R, R)$.

Предположим, что все корни $\tau_{k}$ многочлена $P(x)$ простые и вещественные. Положим $\alpha=\max \left\{\left|\tau_{k}\right|: 1 \leqslant k \leqslant m\right\}$ и обозначим символом $R_{\alpha}$ внутренность ромба с вершинами в точках $(-R, 0),\left(0, \frac{R}{\alpha}\right),(R, 0)$ и $\left(0,-\frac{R}{\alpha}\right)$. Пусть $C^{\infty}\left(R_{\alpha}\right)$ - пространство $Ф$ реше комплекснозначных бесконечно дифференцируемых в $R_{\alpha}$ функций $g\left(x_{1}, x_{2}\right)$.

Имеем $\mu_{j}\left(\lambda_{k, i}\right)=\tau_{j} \lambda_{k, i}+\nu_{j}\left(\lambda_{k, i}\right)$, где при $i=1 \quad k=0,1,2, \ldots$ и при $i=2$ $k=1,2, \ldots$ Так как в данном случае уравнение (1) обладает сильной асимптотикой, то

$$
\sup _{z \in \mathbb{C}}\left|\nu_{j}(z)\right|<\infty, \quad j=0,1, \ldots, m-1 .
$$

Если по разложениям (15) (т.е. разложениям (6)) составить вышеуказанным способом соответствуюший ряд (5), в котором $z_{1}=x_{1}, z_{2}=x_{2}$, то, используя (16), легко установить, что его сумма $w_{0}\left(x_{1}, x_{2}\right)$ принадлежит пространству $C^{\infty}\left(R_{\alpha}\right)$. Обратно, если $w\left(x_{1}, x_{2}\right) \in C^{\infty}\left(R_{\alpha}\right)$, то

$$
\forall j \leqslant m-\left.1 \quad \frac{\partial^{j} w\left(x_{1}, x_{2}\right)}{\partial x_{2}^{j}}\right|_{x_{2}=0} \in C^{\infty}(-R, R) .
$$

В итоге с помощью тех же рассуждений, как и выше, приходим к такому результату. 
Теорема 9. Пусть $0<R<\infty$. Пусть, далее, $\rho_{2}=1$, и пусть все корни многочлена $P(x)$ простые и вещественнье. Тогда следуюшие утверхсдения равносильны:

а) начальные функции $\varphi_{j}(0 \leqslant j \leqslant m-1)$ принадлежст пространству $C^{\infty}(-R, R)$

б) существует решение з.К. (1), (2), представимое в виде абсолютно схо-

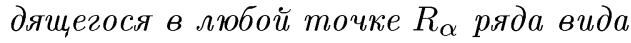

$$
\begin{aligned}
w_{0}\left(x_{1}, x_{2}\right)= & \sum_{j=1}^{N} \sum_{s=0}^{p_{j}-1} \sum_{k=0}^{\infty} a_{j, s}^{(k)}\left(x_{2}\right)^{s} \exp \left[\lambda_{k, 1} x_{1}+\mu_{j}\left(\lambda_{k, 1}\right) x_{2}\right] \\
& +\sum_{j=1}^{N} \sum_{s=0}^{p_{j}-1} \sum_{k=1}^{\infty} b_{j, s}^{(k)}\left(x_{2}\right)^{s} \exp \left[\lambda_{k, 2} x_{1}+\mu_{j}\left(\lambda_{k, 2}\right) x_{2}\right],
\end{aligned}
$$

коэффициенты этого ряда определяются единственным образом по коэффичиентам разложений начальных функций по системе (15), а его сумма $w\left(x_{1}, x_{2}\right)$ принадлежит $C^{\infty}\left(R_{\alpha}\right)$.

Нетрудно получить аналогичный результат для случая, когда все корни $P(x)$ простые и либо все чисто мнимые, либо аргументы любых двух корней совпадают или отличаются на $\pi$. В первом случае строится решение з.К. (1), (2) из пространства комплекснозначных функций, бесконечно дифференцируемых на многообразии $R_{\alpha}^{i}$ всех точек $\left(x_{1}, i y\right)$ таких, что $\left(x_{1}, y\right) \in R_{\alpha}$.

В $\S 4-6$ эффективно (в виде ряда (5)) строилось решение з.К. (1), (2) с определенными свойствами (определенной гладкостью по $z_{1}$ и $z_{2}$ ). При этом единственность решения з.К. (1), (2) в рассматриваемых классах, как правило, не предполагалась, и поэтому о свойствах других возможных решений ничего сказать было нельзя. Если же "нулевая" з.К. (1), (2) с $\varphi_{j}=0, j=0, \ldots, m-1$, имеет только нулевое решение в рассматриваемом классе, то мы получаем описание свойств любого решения з.К. Например, когда $\rho_{2}=1$ и начальные данные $\varphi_{j}$ аналитичны в выпуклой области $\mathscr{G}_{0}$, содержащей начало координат, дополнительное предположение о единственности "нулевой” з.К. в классе функций $v\left(z_{1}, z_{2}\right)$, аналитических в начале координат, приводит (с учетом того, что $\left.(0,0) \in \mathscr{P}_{0}\right)$ к равносильности таких утверждений:

1) $\varphi_{j} \in \mathscr{H}\left(\mathscr{G}_{0}\right), j=0,1, \ldots, m-1$

2) любое аналитическое в окрестности начала координат решение з.К. (1), (2) аналитично в $\mathscr{P} \mathscr{G}_{0}$.

Этот результат дополняет теорему 7.

В ситуации, рассмотренной в теореме 8, достаточно предположить, что "нулевая" з.К. для (1) имеет лишь нулевое решение в классе $\mathscr{H}\left(\mathbb{C}^{2}\right)$ всех цельх функций, чтобы получить, что при этом предположении каждое из утверждений 1)-2) теоремы 8 равносильно такому:

3) любое решение з.К. (1), (2) из класса $\mathscr{H}\left(\mathbb{C}^{2}\right)$ принадлежит пространству $\mathscr{E}\left[\delta, \sigma ; \tau_{0}\right]$.

Аналогичные дополнения можно сделать и к другим доказанным ранее теоремам из $§ 4-6$. 
В связи с этим представляет интерес выяснение, когда же з.К. для уравнения (1) с нулевыми начальными условиями

$$
\left.\frac{\partial^{j} w\left(z_{1}, z_{2}\right)}{\partial z_{2}^{j}}\right|_{z_{2}=0}=0, \quad j=0,1, \ldots, m-1
$$

имеет в том или ином классе функций $v\left(z_{1}, z_{2}\right)$ только нулевое решение.

Опишем простейший, но достаточно важный результат в этом направлении. Пусть в (1) $l_{m}=0$, т.е. уравнение (1) имеет такой вид:

$$
\frac{\partial^{m} w\left(z_{1}, z_{2}\right)}{\partial z_{2}^{m}}=-\sum_{j=0}^{m-1} \sum_{s=0}^{l_{j}} a_{s, j} \frac{\partial^{s+j} w\left(z_{1}, z_{2}\right)}{\partial z_{1}^{s} \partial z_{2}^{j}}
$$

(суммирование ведется по тем $j \leqslant m-1$, для которых $a_{l_{j}, j} \neq 0$ ).

Пусть $\mathscr{F}_{1} \subseteq \mathbb{C}$ и $T$ - класс функций $u\left(z_{1}, z_{2}\right)$, имеющих непрерывные (по совокупности переменных ) на $\mathscr{F}_{1} \times K_{r}$ частные производные всех тех порядков, которые имеются в уравнении (1), и таких, что $\forall z_{1} \in \mathscr{F}_{1}$ функция $u\left(z_{1}, z_{2}\right)$ аналитична по $z_{2}$ в $K_{r}(r=r(u))$. Используя метод, примененный в [17] при доказательстве леммы 1 , получаем, что

$$
\begin{gathered}
\forall n \geqslant 0, \forall k \leqslant \beta=\max \left\{l_{j}: 0 \leqslant j \leqslant m-1\right\}, \forall z_{2} \in K_{r}, \forall z_{1} \in \mathscr{F}_{1}, \forall w \in T \\
\frac{\partial^{n+k} w\left(z_{1}, z_{2}\right)}{\partial z_{1}^{k} \partial z_{2}^{n}}=\frac{\partial^{n+k} w\left(z_{1}, z_{2}\right)}{\partial z_{2}^{n} \partial z_{1}^{k}} .
\end{gathered}
$$

Полагая в (18) $z_{2}=0$, получаем, что $\left.\frac{\partial^{m} w\left(z_{1}, z_{2}\right)}{\partial z_{2}^{m}}\right|_{z_{2}=0}=0$. Дифференцируя равенство (18) при $z \in \mathscr{F} 1, z_{2} \in K_{r}$ по $z_{2}$ и полагая затем $z_{2}=0$, найдем, с учетом (19), что $\left.\frac{\partial^{m+1} w\left(z_{1}, z_{2}\right)}{\partial z_{2}^{m+1}}\right|_{z_{2}=0}=0 \forall z_{1} \in \mathscr{F}_{1}$. Вообще, $\left.\frac{\partial^{n} w\left(z_{1}, z_{2}\right)}{\partial z_{2}^{n}}\right|_{z_{2}=0}=0 \forall n \geqslant 0$, $\forall z_{1} \in \mathscr{F}_{1}$. Отсюда $w\left(z_{1}, z_{2}\right) \equiv 0$ в $\mathscr{F}_{1} \times K_{r}$.

Заметим, что мы не предполагали выполнение условий $l_{j}+j \leqslant m$ при $0 \leqslant j \leqslant$ $m-1$, т.е. уравнение (3) не обязательно является уравнением типа Ковалевской.

Отметим два важных примера уравнений вида (1), для которых $\rho_{2}=1$, а именно волновое уравнение $\frac{\partial^{2} w}{\partial z_{2}^{2}}-\frac{\partial^{2} w}{\partial z_{1}^{2}}=0$ и уравнение Лапласа $\frac{\partial^{2} w}{\partial z_{1}^{2}}+\frac{\partial^{2} w}{\partial z_{2}^{2}}=0$. Читатель без труда сформулирует конкретные следствия о з.К. для этих уравнений, вытекающие из полученных в этом параграфе общих результатов (эти следствия приведены в $[4, \S 6])$.

Приведенные в настоящем параграфе результаты показывают, что в случае $\rho_{2}=$ 1 характер гладкости решения з.К. (1), (2) по переменньм $z_{1}$ и $z_{2}$ примерно один и тот же: решение при фиксированном значении одной переменной принадлежит (по второй переменной) функциональному пространству той же природы (шкалы пространств), что и по первой переменной (при фиксированной второй), например, согласно теореме $8[\delta, \sigma]$ и $\left[\delta, \sigma \tau_{0}\right]$ при $1<\delta<\infty, 0 \leqslant \sigma<\infty$ и т.д. Поэтому будем говорить, что в случае $\rho_{2}=1$ решение з.К. (1), (2) равногладко по обеим переменным. 


\section{$\S 7$. Случай, когда $\rho_{2}>1$}

Рассмотрим последний возможный случай, когда $\rho_{2}>1$. Предположим, что начальные функции $\varphi_{j}\left(z_{1}\right), j=0, \ldots, m-1$, принадлежат пространству $[\nu, \sigma]$, где $\nu=\frac{\rho_{2}}{\rho_{2}-1}, 0 \leqslant \sigma<\infty$. Выберем в $[\nu, \sigma] \mathrm{AПС} \mathscr{E}_{\Lambda}:=\left(\exp \lambda_{k} z_{1}\right)_{k=1}^{\infty}$ так, чтобы $\lim _{k \rightarrow \infty}\left|\lambda_{k}\right|^{-1 / \nu} \ln k=0$.

Пусть (6) - абсолютно сходящиеся в $[\nu, \sigma]$ разложения начальных функций по системе $\mathscr{E}_{\Lambda}$. Тогда (см. начало $\left.\S 5\right)$

$$
\limsup _{k \rightarrow \infty}\left[\left|\lambda_{k}\right|^{-\rho_{2}} \ln \left|b_{k, j}\right|+\delta\right] \leqslant 0
$$

где $\delta\left(\rho_{2}\right)^{\rho_{2}}(\sigma)^{\rho_{2}-1}=\left(\rho_{2}-1\right)^{\rho_{2}-1}$, если $\sigma>0$, и $\delta=+\infty$ при $\sigma=0$.

Составим описанным в $\S 3$ способом ряд (5). Из оценки (9) следует, что для коэффициентов этого ряда справедливо такое же неравенство

$$
\limsup _{k \rightarrow \infty}\left[\left|\lambda_{k}\right|^{-\rho_{2}} \ln \left|a_{j, s}^{(k)}\right|+\delta\right] \leqslant 0, \quad 0 \leqslant s \leqslant p_{j}-1, \quad 1 \leqslant j \leqslant N .
$$

Зафиксируем какие-либо $z_{1} \in \mathbb{C}, j \leqslant N, s \leqslant p_{j}-1$ иположим $d_{j, s}^{(k)}=a_{j, s}^{(k)} \exp \lambda_{k} z_{1}$. Имеем $\lim _{k \rightarrow \infty}\left|\mu_{j, k}\right|\left|\lambda_{k}\right|^{-\rho_{2}}=\left|\tau_{j}\right|$, где $\mu_{j, k}=\mu_{j}\left(\lambda_{k}\right)$. Тогда если $\tau_{j} \neq 0$, то

$$
\limsup _{k \rightarrow \infty}\left|\mu_{j, k}\right|^{-1} \ln \left|d_{j, s}^{(k)}\right|=\limsup _{k \rightarrow \infty}\left|\tau_{j}\right|^{-1} \ln \left|a_{j, s}^{(k)}\right| \cdot\left|\lambda_{k}\right|^{-\rho_{2}}
$$

откуда

$$
\underset{k}{\limsup }\left[\ln \left|d_{j, s}^{(k)}\right| \cdot\left|\mu_{j, k}\right|^{-1}+\delta\left|\tau_{j}\right|^{-1}\right] \leqslant 0
$$

Следовательно, при любом фиксированном $z_{1} \in \mathbb{C}$ каждый ряд

$$
\sum_{k=1}^{\infty} a_{j, s}^{(k)} \exp \lambda_{k} z_{1} \exp \mu_{j, k} z_{2}
$$

сходится абсолютно в $\mathscr{H}\left(K_{\delta /\left|\tau_{j}\right|}\right)$. Если же $\tau_{j}=0$ или $\sigma=0$, то $\lim _{k \rightarrow \infty} \frac{\ln \left|d_{j, s}^{(k)}\right|}{\left|\mu_{j, k}\right|}=$ $-\infty$, и каждый из этих рядов сходится абсолютно в $\mathscr{H}(\mathbb{C})$.

Зафиксируем теперь любое $r \in(0,+\infty)$, если $\tau_{j}=0$ или если $\sigma=0$, и любое $r$ из $\left(0, \delta\left|\tau_{j}\right|^{-1}\right)$, если $\tau_{j} \neq 0, \sigma \neq 0$. Положим $b_{j, s}^{(k)}=a_{j, s}^{(k)} \exp \mu_{j, s} z_{2}$. Имеем при $\sigma>0,\left|z_{2}\right| \leqslant r$

$$
\underset{k}{\limsup }\left|\lambda_{k}\right|^{-\rho_{2}} \ln \left|b_{j, s}^{(k)}\right| \leqslant-\delta+\left|z_{2}\right|\left|\tau_{j}\right| \leqslant-\delta+r \tau
$$

где $\tau=\max \left\{\left|\tau_{j}\right|: 1 \leqslant j \leqslant m\right\}$. Следовательно, если $\left|z_{2}\right| \leqslant r<\frac{\delta}{\tau}$, то ряд $\sum_{k=1}^{\infty} b_{j, s}^{(k)} \exp \lambda_{k} z_{1}$ сходится абсолютно в $[\nu, \delta(r)]$, где $\left[\rho_{2}(\delta-r \tau)\right]^{1 / \rho_{2}}=[\nu \delta(r)]^{-1 / \nu}$, т.е. $\delta(r)=\frac{1}{\nu}\left[\rho_{2}(\delta-r \tau)\right]^{-\nu / \rho_{2}}$. Заметим, что $\delta(0)=\sigma$ (напомним, что $\delta \rho_{2}=$ $\left.(\sigma \nu)^{1-\rho_{2}}\right)$. Если же $\sigma=0$, то

$$
\begin{aligned}
\underset{k}{\limsup }\left|\lambda_{k}\right|^{-\rho_{2}} \ln \left|b_{j, s}^{(k)}\right| \leqslant & \limsup _{k}\left|\lambda_{k}\right|^{-\rho_{2}} \ln \left|a_{j, s}^{(k)}\right| \\
& +\limsup _{k}\left|\mu_{j, k}\right|\left|z_{2}\right|\left|\lambda_{k}\right|^{-\rho_{2}}=-\infty
\end{aligned}
$$


и ряд $\sum_{k=1}^{\infty} b_{j, s}^{(k)} \exp \lambda_{k} z_{1}$ сходится абсолютно в $[\nu, 0]$. Положим $\eta(r)=\delta(r)$, если $\sigma>0 ; \eta(r)=0$, если $\sigma=0 ; \eta=\delta$, если $\sigma>0 ; \eta=+\infty$, если $\sigma=0$. Сформулируем полученный результат.

TEOPEMA 10. Пусть $\rho_{2}>1$, nyсmb $\tau:=\max \left\{\left|\tau_{j}\right|: 1 \leqslant j \leqslant m\right\}, \quad \nu:=\frac{\rho_{2}}{\rho_{2}-1}$, $\delta:=\frac{1}{\rho_{2}}(\sigma \nu)^{1-\rho_{2}}$. Предположим, что $\varphi_{j} \in[\nu, \sigma]$ при некотором $\sigma \in[0,+\infty) u$ $j=0, \ldots, m-1$.

Тогда существует решение з.К. (1), (2) $w_{0}\left(z_{1}, z_{2}\right)$, представимое рядом (5), который сходится абсолютно $\left(\right.$ по $\left.z_{2}\right)$ в $\mathscr{H}\left(K_{\eta / \tau}\right)$ при любом фиксированном $z_{1} \in \mathbb{C}$ и сходится абсолютно в $\left[\nu, \eta\left(\left|z_{2}\right|\right)\right]$ при любом фиксированном $z_{2} \in$ $K_{\eta / \tau}$.

Введем пространство $\mathscr{E}[\nu, \sigma ; \eta, \tau]$ функций $y\left(z_{1}, z_{2}\right)$ из $\mathscr{H}\left(\mathbb{C} \times K_{\eta / \tau}\right)$ таких, что $\forall n \geqslant 1$

$$
\|y\|_{n}=\sup \left\{\left|y\left(z_{1}, z_{2}\right)\right| \exp \left[-\left(\eta\left(r_{n}\right)+\frac{1}{n}\right)\left|z_{1}\right|^{\nu}\right]: z_{1} \in \mathbb{C},\left|z_{2}\right| \leqslant r_{n}\right\}<\infty,
$$

где $\left\{r_{n}\right\}_{n=1}^{\infty}-$ произвольно зафиксированная последовательность положительных чисел такая, что $r_{n} \uparrow \frac{\eta}{\tau}$.

Используя примерно те же выкладки, что и при доказательстве теоремы 10 , получаем, что в предположениях этой теоремы ряд (5), представляющий решение 3.К. (1), (2), сходится абсолютно в пространстве $\Phi$ реше $\mathscr{E}[\nu, \sigma ; \eta, \tau]$.

СЛЕДСТВИЕ 1. Пусть $\rho_{2}, \tau, \nu, \eta, \delta$ - me жее, что и в теореме 10, и пусть $\varphi \in[\nu, \sigma]$ при $j=0, \ldots, m-1$ и при некотором $\sigma \in[0,+\infty)$. Тогда существует решение з.К. (1), (2)из пространства $\left(\mathscr{H}(\mathbb{C}) ; \mathscr{H}\left(K_{\eta / \tau}\right)\right)$.

СЛЕДСТВИЕ 2. Пусть $\rho_{2}, \tau, \nu, \delta$ - mе же, что и в теореме 10, и пусть $\varphi \in[\nu, \sigma]$ при $j=0, \ldots, m-1$ и при некотором $\sigma \in[0,+\infty)$. Тогда существует решение з.К. (1), (2) из пространства $\mathscr{E}[\nu, \sigma ; \eta, \tau]$.

Из определения пространства $\mathscr{E}[\nu, \sigma ; \eta, \tau]$ следует, что если $y\left(z_{1}, z_{2}\right) \in \mathscr{E}[\nu, \sigma ; \eta, \tau]$, то $y \in \mathscr{H}\left(\mathbb{C} \times K_{\eta / \tau}\right)$; более того, если положить $r_{1}=0<r_{1}<\cdots<r_{n} \uparrow \frac{\eta}{\tau}$ и учесть, что $\nu \eta(0)=\nu \delta(0)=\left(\rho_{2} \delta\right)^{\frac{1}{1-\rho_{2}}}=\nu \sigma$, то $y\left(z_{1}, 0\right) \in[\nu, \sigma]$. Далее, как легко показать, если $y \in \mathscr{E}[\nu, \sigma ; \eta, \tau]$, то $\forall k>1 \frac{\partial^{k} y}{\partial z_{2}^{k}} \in \mathscr{E}[\nu, \sigma ; \eta, \tau]$, откуда $\left.\frac{\partial^{k} y}{\partial z_{2}^{k}}\right|_{z_{2}=0} \in[\nu, \sigma], k=1,2, \ldots$

Из следствия 2 получаем

СЛЕДСТВИЕ 3. Пусть $\rho_{2}, \tau, \nu, \eta, \delta$ - mе жее, что и в теореме 10, и пусть $\varphi \in \mathscr{H}(\{0\}), j=0, \ldots, m-1$. Для того чтобы з.К. (1), (2) имела решение в $\mathscr{E}(\nu, \sigma ; \eta, \tau)$, необходимо и достаточно, чтобы $\varphi_{j} \in[\nu, \sigma], j=0, \ldots, m-1$.

Пусть теперь $1<\beta<\nu=\rho_{2} /\left(\rho_{2}-1\right)$, а $\delta_{2}$ определяется равенством $\delta_{2}(\beta-$ $\left.\beta \rho_{2}+\rho_{2}\right)=\beta$. Введем пространство Фреше

$$
\begin{aligned}
& \mathscr{E}_{0}\left[\delta_{2}, \beta\right] \\
& =\left\{y\left(z_{1}, z_{2}\right) \in \mathscr{H}\left(\mathbb{C}^{2}\right)\left|\|y\|_{n}=\sup _{z_{1}, z_{2} \in \mathbb{C}}\right| y\left(z_{1}, z_{2}\right) \mid \exp \left[-\frac{1}{n}\left(\left|z_{2}\right|^{\delta_{2}}+\left|z_{1}\right|^{\beta}\right)\right]<\infty,\right. \\
& n=1,2, \ldots\} .
\end{aligned}
$$


Используя те же рассуждения, как в случае теоремы 10, приходим к такому результату.

TЕорема 11. Пусть $\rho_{2}>1,1<\beta<\nu$, и пусть $\varphi_{j} \in[\beta, 0], j=0, \ldots, m-1$. Тогда существует решение з.К. (1), (2), представимое в виде ряда (5), который сходится абсолютно в $\left([\beta, 0] ;\left[\delta_{2}, 0\right]\right)$. Более того, этот ряд сходится абсолютно в пространстве $\mathscr{E}_{0}\left[\delta_{2}, \beta\right]$.

СЛЕДСТВИЕ 1. Пусть $\rho_{2}, \beta, \nu, \delta_{2}-$ me же, что и в теореме 11, и пусть $\varphi_{j} \in[\beta, 0], j=0, \ldots, m-1$. Тогда существует решение $w\left(z_{1}, z_{2}\right)$ з.K. (1), (2) из пространства $\left([\beta, 0] ;\left[\delta_{2}, 0\right]\right)$.

СлЕДСТВИЕ 2. В предположениях теоремы 11 з.К. (1), (2) разрешима в пространстве Фреше $\mathscr{E}_{0}\left[\delta_{2}, \beta\right]$.

Пусть $\mathscr{H}(\overline{\{0\}})$ - множество всех функций, аналитических в окрестности начала координат (своей для каждой функции).

СлЕДСТВИЕ 3. Пусть $\rho_{2}, \beta, \nu, \delta_{2}-$ me же, что и в теореме 11, и пусть $\varphi_{j} \in \mathscr{H}(\overline{\{0\}}), j=0, \ldots, m-1$. Для того чтобы з.К. (1), (2) была разрешима в пространстве $\mathscr{E}_{0}\left[\delta_{2}, \beta\right]$, необходимо и достаточно, чтобы $\varphi_{j} \in[\beta, 0], j=$ $0, \ldots, m-1$.

Покажем теперь, что полученные до сих пор результаты о разрешимости з.К. (1), (2) позволяют установить довольно общую теорему единственности. Предварительно обозначим символом $\mathscr{E}_{b}$ векторное пространство целых функций $f\left(z_{1}, z_{2}\right)$ таких, что при любом фиксированном $z_{2} \in \mathbb{C} f\left(z_{1}, z_{2}\right) \in[b, 0]$, а при любом $z_{1} \in \mathbb{C}$ $f\left(z_{1}, z_{2}\right)$ - целая функция конечного порядка (по $\left.z_{2}\right)$.

ТЕОРемА 12. Следующие утверждения равносильны:

1) $l_{m}=0$

2) нулевая з.К. (1), (17) имеет только тривиальное решение в классе функиий из $\mathscr{H}(\overline{(0,0)})$;

3) з.К. (1), (17) имеет только тривиальное решение в $\mathscr{H}\left(\mathbb{C}^{2}\right)$;

4) з.K. (1), (17) имеет лишь нулевое решение в $\mathscr{E}_{b}$ при любом $b>1$;

5) з.K. (1), (17) имеет только тривиальное решение в $\mathscr{E}_{b}$ при некотором $b>1$.

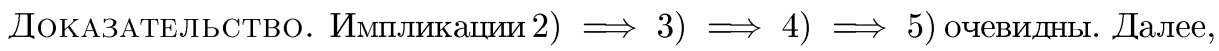
импликация 1) $\Longrightarrow 2$ ) была установлена в конце $\S 6$. Нам осталось доказать импликацию 5) $\Longrightarrow 1$ ). Допустим, что $l_{m}>0$. Пусть $\alpha-$ какой-нибудь корень уравнения $P_{m}(x):=\sum_{s=0}^{l_{m}} a_{s, m} x^{s}$. Рассмотрим уравнение

$$
0=M_{1} w, \quad \text { где } \quad M_{1} w:=M \frac{\partial w}{\partial z_{2}}=\sum_{j=0}^{m} \sum_{s=0}^{l_{j}} a_{s, j} \frac{\partial^{s+j+1}}{\partial z_{1}^{s} \partial z_{2}^{j+1}} .
$$

Пусть $\widetilde{\rho}_{2}-$ кор. инд. этого уравнения. Зададим такие начальные условия для уравнения (20):

$$
\left.\frac{\partial^{k} w\left(z_{1}, z_{2}\right)}{\partial z_{2}^{k}}\right|_{z_{2}=0}=0, k=0,1, \ldots, m-1 ;\left.\quad \frac{\partial^{m} w\left(z_{1}, z_{2}\right)}{\partial z_{2}^{m}}\right|_{z_{2}=0}=\exp \left(\alpha z_{1}\right)
$$


Применяя теорему 1 , если $\widetilde{\rho}_{2} \leqslant 0$, теорему 4 , если $0<\widetilde{\rho}_{2}<1$, теорему 8 при $\widetilde{\rho}_{2}=1$ и, наконец, теорему 11 , если $\widetilde{\rho}_{2}>1$, находим, что $\forall b>1$ в $\mathscr{E}_{b}$ сушествует решение $w_{0}$ з.К. (20), (21). Тогда $\forall z_{1}, z_{2} \in \mathbb{C}$

$$
\begin{aligned}
0 & =\sum_{j=0}^{m} \sum_{s=0}^{l_{j}} a_{s, j} \int_{0}^{z_{2}} \frac{\partial^{s+j+1} w_{0}\left(z_{1}, t\right)}{\partial z_{1}^{s} \partial t^{j+1}} d t \\
& =\sum_{j=0}^{m} \sum_{s=0}^{l_{j}}\left\{a_{s, j} \frac{\partial^{s}}{\partial z_{1}^{s}}\left[\frac{\partial^{j} w_{0}\left(z_{1}, z_{2}\right)}{\partial z_{2}^{j}}\right]-\left.\frac{\partial^{s}}{\partial z_{1}^{s}} \frac{\partial^{j} w_{0}\left(z_{1}, z_{2}\right)}{\partial z_{2}^{j}}\right|_{z_{2}=0}\right\} \\
& =M w_{0}-P_{m}(\alpha) \exp \alpha z_{1}=M w_{0} .
\end{aligned}
$$

Таким образом, $w_{0}$ - нетривиальное решение з.К. (1), (17) из $\mathscr{E}_{b}$, и утверждение 5) не имеет места. Теорема доказана.

Возникает естественный вопрос о возможности обрашения теорем 10, 11 и их первых следствий. Начнем с теоремы 11 и установим такое её частичное обращение.

TeOPEMA 13. a) Пусть $\rho_{2}>1, \quad \nu=\frac{\rho_{2}}{\rho_{2}-1}$, u nyсmb $\varphi_{j} \in \mathscr{H}(\{\overline{0}\}), \quad j=$ $0, \ldots, m-1$

b) Пусть, далее, $r_{0}$ - радиус наибольшего круга с чентром в начале координат, в котором аналитичны все начальные функиии $\varphi_{j}$, и пусть $\mathscr{E}_{\Lambda}-A П C$ в $\mathscr{H}\left(K_{r_{0}}\right)$ такая, что $\lim _{n \rightarrow \infty} \frac{\ln n}{\lambda_{n}}=0$.

с) Допустим, что существует к. $\mathscr{E}_{\Lambda}$-ряд (5) такой, что при некотором фиксированном $z_{1} \in \mathbb{C}$ он сходится абсолютно в $\mathscr{H}\left(K_{R}\right), 0<R<\infty$.

Тогда $\varphi_{j} \in[\nu, \sigma]$, где $\sigma=\left(\rho_{2}-1\right)(\alpha R)^{\frac{1}{1-\rho_{2}}} \cdot\left(\rho_{2}\right)^{\frac{\rho_{2}}{1-\rho_{2}}} \quad u \alpha=\min \left\{\left|\tau_{l}\right|: 1 \leqslant\right.$ $l \leqslant m\}$.

ДокАЗАТЕЛЬСТво. Положим $d_{j, s}^{(k)}=a_{j, s}^{(k)} \exp \lambda_{k} z_{1}$, где $s=0,1, \ldots, p_{j}-1$; $j=1,2, \ldots, N$. Из предположений теоремы следует, что каждый ряд $\sum_{k=1}^{\infty} d_{j, s}^{(k)} \times$ $\exp \mu_{j, k} z_{2}$ сходится абсолютно в $\mathscr{H}\left(K_{R}\right)$. Отсюда при тех же $s$ и $j$

$$
\limsup _{k}\left[\left|\mu_{j, k}\right|^{-1} \ln \left|d_{j, s}^{(k)}\right|+R\right] \leqslant 0,
$$

или, что все равно (в силу того, что $\lim _{k}\left|\mu_{j, k}\right| \cdot\left|\lambda_{k}\right|^{-\rho_{2}}=\left|\tau_{j}\right|$ и $\rho_{2}>1$ ),

$$
\underset{k}{\limsup }\left[\left|\lambda_{k}\right|^{-\rho_{2}} \ln \left|a_{j, s}^{(k)}\right|+\left|\tau_{j}\right| R\right] \leqslant 0, \quad 0 \leqslant s \leqslant p_{j}-1, \quad 1 \leqslant j \leqslant N .
$$

Подавно для тех же $s$ и $j$

$$
\underset{k}{\limsup }\left[\left|\lambda_{k}\right|^{-\rho_{2}} \ln \left|a_{j, s}^{(k)}\right|+\alpha R\right] \leqslant 0 .
$$

Из равенств (12) получаем, что при $n=0,1, \ldots, m-1$

$$
\underset{k}{\limsup }\left[\left|\lambda_{k}\right|^{-\rho_{2}} \ln \left|b_{k, n}\right|+\alpha R\right] \leqslant 0 .
$$


Тогда $\forall n \leqslant m-1, \forall z \in K_{r_{0}} \varphi_{n}(z)=\sum_{k=1}^{\infty} b_{k, n} \exp \lambda_{k} z$. Отсюда $\varphi_{n}(z) \in[\nu, \sigma]$, и теорема доказана.

Заметим, что если модули всех корней многочлена $P(x)$ одинаковы, то теорема 13 в этом случае является точным обрашением теоремы 10.

Кроме того, аналогичными выкладками получаем такое точное обращение теоремы 10 в случае, когда $\sigma=0$ :

ТЕОРема 14. Допустим, что выполнены предположсения а), b) теоремьи 13, и пусть существует $\kappa . \mathscr{E}_{\Lambda}$-ряд (5) такой, что при некотором фиксированном $z_{1} \in \mathbb{C}$ этот ряд сходится абсолютно в $\mathscr{H}(\mathbb{C})$. Тогда $\varphi_{j} \in[\nu, 0]$, $j=0,1, \ldots, m-1$.

Точно таким же методом можно получить обращение теоремы 11.

ТЕОРема 15. Пусть выполнены предположения а), b) теоремы 13. Пусть, далее, существует к. $\mathscr{E}_{\Lambda}$-ряд (5) такой, что при некотором фиксированном $z_{1} \in \mathbb{C}$ этот ряд сходится абсолютно в $[\gamma, 0]$, где $1<\gamma<\infty$. Тогда $\varphi_{j} \in$ $\left[\gamma_{1}, 0\right]$, əде $\gamma_{1}\left(\rho_{2} \gamma-\gamma-1\right)=\rho_{2} \gamma$

Что же касается первых следствий теорем 10, 11, то их обращение оказалось более трудным и потребовало довольно большой дополнительной информации о кор. инд., которая излагается в следующем параграфе. Причина трудностей, возникающих при обращении этих следствий, заключается в том, что мы предполагаем в них лишь наличие решения с определенной гладкостью по $z_{2}$ (при фиксированном $z_{1}$ ), но ничего не знаем о его структуре (например, возможности его представления в виде ряда (5)).

\section{§ 8. Дополнительные сведения о кор. индексах}

Напомним, что в уравнении (1) рассматриваются только те $j \leqslant m$, для которых $a_{l_{j}, j} \neq 0$. Обозначим символом $\mathscr{N}_{2}(P)$ множество всех точек с целочисленными координатами вида $(j, k)$, где $0 \leqslant j \leqslant l_{k}, 0 \leqslant k \leqslant m$ и $a_{j, k} \neq 0$. В частности, $\left(l_{m}, m\right) \in \mathscr{N}_{2}(P)$ и $\left(l_{k}, k\right) \in \mathscr{N}_{2}(P)$, если $k \leqslant m$ и $a_{l_{k}, k} \neq 0$. Очевидно, что ордината точки $\left(l_{k}, k\right)$ из $\mathscr{N}_{2}(P)$ принадлежит введенному в $\S 1$ множеству $\mathscr{N}_{0}$ тогда и только тогда, когда эта точка лежит на прямой $L: x-l_{m}=-\rho_{2}(y-m)$. Всюду далее в этом параграфе предполагается, что $\rho_{2}>0$, т.е. $\exists k \leqslant m-1: l_{k}>l_{m}$.

Проведем из точки $\left(l_{m}, m\right)$ луч, проходящий через начало координат, и будем вращать его вокруг точки $\left(l_{m}, m\right)$ против часовой стрелки до тех пор, пока на нем не окажется хотя бы одна точка $(j, k)$ из $\mathscr{N}_{2}(P)$, отличная от $\left(l_{m}, m\right)$, а все остальные точки $\mathscr{N}_{2}(P)$ лежат на этом луче или ниже его. Нетрудно убедиться в том, что такое предельное положение луча проходит по прямой $L$. Пусть $k_{0}=$ $\min \left\{k \in N_{0}:\left(l_{k}, k\right) \in L\right\}$. Тогда ниже точки $\left(l_{k_{0}}, k_{0}\right)$ на $L$ нет точек из $\mathscr{N}_{2}(P)$.

Перепишем характеристический многочлен уравнения (1):

$$
Q(\lambda, \mu)=\sum_{j=0}^{m} \mu^{j} \sum_{s=0}^{l_{j}} a_{s, j} \lambda^{s}=\sum_{j=0}^{m} \sum_{s=0}^{l_{j}} a_{s, j} \mu^{j} \lambda^{s}
$$


где суммирование во втором представлении ведется по тем $s$ и $j$, для которых $(s, j) \in \mathscr{N}_{2}(P)$, в таком виде:

$$
Q(\lambda, \mu)=\sum_{s=0}^{p} \lambda^{s} \sum_{j=0}^{\nu_{s}} a_{s, j} \mu^{j}
$$

В равенстве (22) суммирование ведется по тем $s$, для которых $a_{s, \nu_{s}} \neq 0$. Очевидно, что

$$
p=\max \left\{r:(r, s) \in \mathscr{N}_{2}(P)\right\}=\max \left\{l_{k}: 0 \leqslant k \leqslant m,\left(l_{k}, k\right) \in \mathscr{N}_{2}(P)\right\}
$$

При этом так как $\rho_{2}>0$, то $p=\max \left\{l_{k}: 0 \leqslant k<m,\left(l_{k}, k\right) \in \mathscr{N}_{2}(P)\right\}$.

Введем характеристику, аналогичную $\rho_{2}$ :

$$
\rho_{1}:=\max \left\{\frac{\nu_{s}-\nu_{p}}{p-s}: 0 \leqslant s<p, a_{s, \nu_{s}} \neq 0\right\} .
$$

Будем также называть ее индексом корреляции уравнения (1) по переменной $z_{1}$ (кор. индексом по $z_{1}$ ).

ТЕОРЕМа 16. Если $\rho_{2}>0$, то $\rho_{1} \rho_{2} \geqslant 1$. При этом $\rho_{1}=1 / \rho_{2}$ тогда и только тогда, когда правее самой нижней из лежащих на прямой $L$ точек множества $\mathscr{N}_{2}(P)$ нет ни одной точки множества $\mathscr{N}_{2}(P)$.

Заметим, что $l_{m}<p$ и $\nu_{l_{m}}=m$, откуда $\frac{m-\nu_{p}}{p-l_{m}} \leqslant \rho_{1}$. Далее, $l_{\nu_{p}}=p, \nu_{p} \leqslant m$. Допустим, что $\nu_{p}=m$. Но тогда $p=l_{\nu_{p}}=l_{m}$, что невозможно. Итак, $\nu_{p}<m$ и, следовательно, $\rho_{2} \geqslant \frac{l_{\nu_{p}}-l_{m}}{m-\nu_{p}}=\frac{p-l_{m}}{m-\nu_{p}}$. Отсюда $\rho_{1} \geqslant \frac{m-\nu_{p}}{p-l_{m}} \geqslant \frac{1}{\rho_{2}}$.

Пусть теперь правее прямой $h=l_{k_{0}}$ нет точек из $\mathscr{N}_{2}(P)$. Простое геометрическое рассмотрение показывает, что тогда $p=l_{k_{0}}$. Но так как $\nu_{l_{k}}=k$, то $\nu_{p}=k_{0}$. Далее, $\forall s<p\left(s, \nu_{s}\right) \in \mathscr{N}_{2}(P) \Longleftrightarrow a_{s, \nu_{s}} \neq 0$. Если $\left(s, \nu_{s}\right)$ из $\mathscr{N}_{2}(P)$ лежит ниже $L$, то $\frac{s-l_{m}}{m-\nu_{s}}<\rho_{2}$, откуда $\nu_{s}<m+\frac{l_{m}-s}{\rho_{2}}$. Но тогда

$$
\frac{\nu_{s}-\nu_{p}}{p-s}=\frac{\nu_{s}-k_{0}}{l_{k_{0}}-s}<\frac{m \rho_{2}+l_{m}-s-\rho_{2} k_{0}}{\rho_{2}\left(l_{k_{0}}-s\right)}=\frac{1}{\rho_{2}} .
$$

Если же $\left(s, \nu_{s}\right) \in \mathscr{N}_{2}(P) \cap L$, то $\frac{\nu_{s}-\nu_{p}}{p-s}=\frac{m-k_{0}}{l_{k_{0}}-l_{m}}=\frac{1}{\rho_{2}}$. Следовательно, $\rho_{1} \leqslant \frac{1}{\rho_{2}}$, откуда $\rho_{1} \rho_{2}=1$.

Пусть, обратно, правее прямой $x=l_{k_{0}}$ (но, разумеется, ниже $L$ ) есть хотя бы одна точка из $\mathscr{N}_{2}(P)$. Тогда $p>l_{k_{0}}$ и $p=l_{k_{1}}$, где $0 \leqslant k_{1}<k_{0}$, и $\nu_{p}=k_{1}$. Отсюда

$$
\rho_{1} \geqslant \frac{\nu_{l_{k_{0}}}-\nu_{p}}{p-l_{k_{0}}}=\frac{k_{0}-k_{1}}{l_{k_{1}}-l_{k_{0}}}>\frac{1}{\rho_{2}} .
$$

Возврашаясь к характеристическому многочлену $Q(\lambda, \mu)$, записанному в виде $(22)$, заметим, что в окрестности бесконечно удаленной точки уравнение $Q(\lambda, \mu)=0$ имеет $p$ ветвей $\lambda=\lambda_{k}(\mu)(k=0,1, \ldots, p-1)$ определяемой этим уравнением алгебраической функции, причем эти ветви не обязательно различны. Для любой такой ветви $\lambda(\mu)$ существует конечный $\lim _{\mu \rightarrow \infty} \mu^{-\rho_{1}} \lambda(\mu)=\nu$, где $\nu-$ 
один из корней многочлена $P_{1}(y):=\sum_{s \in \mathcal{N}_{4}} a_{s, \nu_{s}} y^{s}$ и $N_{4}=\{p\} \cup\{s: 0 \leqslant s<p$, $\left.\nu_{s}-\nu_{p}=\rho_{1}(p-s)\right\}$.

Нетрудно проверить, что если $\rho_{1}=\frac{1}{\rho_{2}}$, то любой ненулевой корень $y$ уравнения $P_{1}(y)=0$ представим в виде $y=x^{-\frac{1}{\rho_{2}}}$, где $P(x)=0$, и, обратно, если $P(x)=0$ и $x \neq 0$, то $x=y^{-\rho_{2}}$, где $P_{1}(y)=0$. В частности, если все корни $P$ равны по абсолютной величине, скажем, числу $A \neq 0$, то и все корни $P_{1}$ имеют одну и ту же абсолютную величину $A^{-\frac{1}{\rho_{2}}}$ (и обратно).

В следующем параграфе нам понадобится описание одного подкласса уравнений (1).

ТЕОРема 17. Для уравнения (1) равносильны следующие утвержсдения:

1) $\rho_{2}>1, \rho_{1}=1 / \rho_{2}$ и з.К. для уравнения (1) по переменной $z_{1}$ :

$$
\left.\frac{\partial^{k} w}{\partial z_{1}^{k}}\right|_{z_{1}=0}=0, \quad k=0,1, \ldots, p-1
$$

имеет только нулевое решение в $\mathscr{H}((\overline{0,0}))$;

2) $l_{0}=p>l_{k}, \quad k=1,2, \ldots, m-1 ; \quad p>l_{m}+m ; \quad p \geqslant \frac{m l_{k}-k l_{m}}{m-k}, \quad k=$ $1,2, \ldots, m-1$.

ДокАЗАТЕЛЬСтво. Пусть сначала выполнены условия 1). По теореме 12 $\nu_{p}=0$, что, очевидно, равносильно соотношениям $l_{0}=p>l_{k}, k=1,2, \ldots, m$. Как показано в начале доказательства теоремы 16 , всегда $\rho_{1} \geqslant \frac{m-\nu_{p}}{p-l_{m}} \geqslant \frac{1}{\rho_{2}}$. Так как $\nu_{p}=0$ и $\rho_{1} \rho_{2}=1$, то $l_{m}+m \rho_{2}=m$. Отсюда $p>l_{m}+m$ и $\frac{p-l_{m}}{m}=\rho_{2} \geqslant \frac{l_{k}-l_{m}}{m-k}$, $k=1,2, \ldots, m-1$ (по определению $\rho_{2}$ ).

Пусть, обратно, выполнены условия 2$)$. Тогда $\forall k \leqslant m-1$

$$
\frac{l_{0}-l_{m}}{m}=\frac{p-l_{m}}{m} \geqslant \frac{l_{k}-l_{m}}{m-k} .
$$

Тогда $\rho_{2}=\left(p-l_{m}\right) / m$ и $\rho_{2}>1$. При этом $\{0\} \in \mathscr{N}_{0}$. Прямая $L$ проходит через точки $\left(l_{m}, m\right)$ и $(p, 0)$, а все остальные точки множества $\mathscr{N}_{2}(P)$ принадлежат (замкнутой) трапеции с вершинами в точках $(0, m),\left(l_{m}, m\right),(p, 0)$ и $(0,0)$. Самой нижней из лежаших на $L$ точек множества $\mathscr{N}_{2}(P)$ является точка $(p, 0)$, и правее ее точек из $\mathscr{N}_{2}(P)$ нет. По теореме $16 \rho_{1}=1 / \rho_{2}$. Наконец, из соотношений $l_{0}=p>l_{k}$, $k=1,2, \ldots, m-1$, и $l_{0}=p>l_{m}+m$ следует, что $\nu_{p}=0$, и по теореме 12 3.К. (1), (23) имеет только нулевое решение в $\mathscr{H}((\overline{0,0}))$.

Заметим, что условия 2) теоремы 16 равносильны таким:

$$
l_{0}=p>l_{m}+m ; \quad p m+k l_{m} \geqslant m l_{k}+k p, \quad k=1,2, \ldots, m-1 .
$$

Действительно, последняя (третья) группа условий 2) равносильна последней (второй) группе условий (24). Поэтому условия (24) следуют из 2). Пусть, обратно, выполнены условия (24). Тогда при $k=1,2, \ldots, m-1$

$$
p m+k l_{m} \geqslant m l_{k}+k p>m l_{k}+k l_{m}+k m,
$$


откуда $p>l_{k}+k$.

Попутно установлено, что условие 2) теоремы 17 равносильно также таким, по форме более сильным, условиям:

$$
\begin{gathered}
l_{0}=p>l_{k}+k, \quad k=1,2, \ldots, m ; \\
p m+k l_{m} \geqslant m l_{k}+k p, \quad k=1,2, \ldots, m-1 .
\end{gathered}
$$

\section{§ 9. Случай $\rho_{2}>1$ (продолжение)}

Теперь уже можно заняться обрашением первых следствий теорем 10, 11. Предварительно перепишем уравнение (1) в таком виде:

$$
\sum_{s=0}^{p}, \sum_{j=0}^{\nu_{s}} a_{s, j} \frac{\partial^{s+j} w\left(z_{1}, z_{2}\right)}{\partial z_{1}^{s} \partial_{2}^{j}}=0,
$$

где суммирование ведется по тем $s \leqslant p$, для которых $a_{s, \nu_{s}} \neq 0 ; p=\max \left\{l_{j}: j=\right.$ $0,1, \ldots, m\} ; \nu_{s} \leqslant m, s=0, \ldots, p ; a_{p, \nu_{p}} \neq 0$.

Согласно теореме 12 условие $\nu_{p}=0$ необходимо и достаточно для того, чтобы "нулевая" з.К. (23) для уравнения (26) по переменной $z_{1}$ имела в классе $\mathscr{H}(\overline{(0,0)})$ только тривиальное решение $w\left(z_{1}, z_{2}\right) \equiv 0$. Всюду далее в этом параграфе предполагается, что $\nu_{p}=0$.

Пусть $w_{1}\left(z_{1}, z_{2}\right)$ - любое решение з.К. (1), (2), аналитическое в окрестности начала координат: $\exists r_{1}>0, \exists r_{2}>0: w_{1}\left(z_{1}, z_{2}\right) \in \mathscr{H}\left(K_{r_{1}} \times K_{r_{2}}\right)$. Тогда $\forall k \geqslant 0$

$$
\psi_{k}\left(z_{2}\right):=\left.\frac{\partial^{k} w_{1}\left(z_{1}, z_{2}\right)}{\partial z_{1}^{k}}\right|_{z_{1}=0} \in \mathscr{H}\left(K_{r_{2}}\right) .
$$

Рассмотрим для уравнения (26) з.К. по $z_{1}$ с начальньми условиями:

$$
\left.\frac{\partial^{k} w\left(z_{1}, z_{2}\right)}{\partial z_{1}^{k}}\right|_{z_{1}=0}=\psi_{k}\left(z_{2}\right), \quad k=0,1, \ldots, p-1 .
$$

Предположим еще, что $\rho_{1}<1$. Напомним, что $\rho_{1}<1$, если, в частности, выполнены условия $(25)$ (тогда $\rho_{2}=1 / \rho_{1}>1$ ). Применим теорему 2 , предварительно поменяв в ней местами $z_{1}$ и $z_{2}$. Согласно этой теореме сушествует решение $w_{2}\left(z_{1}, z_{2}\right)$ з.К. (26), (27), представимое в виде ряда

$$
\sum_{j=1}^{N_{1}} \sum_{s=0}^{q_{j}-1} \sum_{k=1}^{\infty} a_{j, s}^{(k)}\left(z_{1}\right)^{s} \exp \left[\mu_{k} z_{2}+\lambda_{j}\left(\mu_{k}\right) z_{1}\right],
$$

где $q_{j}-$ кратность ветви $\lambda_{j}(\mu)$, порожденной многочленом $Q(\lambda, \mu)$, и $\left(\exp \mu_{k} z_{2}\right)_{k=1}^{\infty}-\mathrm{A \Pi C} \mathrm{в} \mathscr{H}\left(K_{r_{2}}\right)$, для которой $\lim _{k} \frac{\ln k}{\left|\mu_{k}\right|}=0$. Ряд (28) сходится абсолютно в пространстве $\mathscr{E}\left(\rho_{1}, r_{2}\right)$ функций $y\left(z_{1}, z_{2}\right)$ из $\mathscr{H}\left(\mathbb{C} \times K_{r_{2}}\right)$ таких, что

$$
\begin{gathered}
\forall r \in\left(0, r_{2}\right), \quad \forall n \geqslant 1 \\
|y|_{r, n}:=\sup \left\{\frac{\left|y\left(z_{1}, z_{2}\right)\right|}{\exp \left[\sigma(r)+\frac{1}{n}\right]|z|^{\frac{1}{1-\rho_{1}}}}|| z_{2} \mid \leqslant r, z_{1} \in \mathbb{C}\right\}<\infty,
\end{gathered}
$$


где $\sigma(r)=\left(1-\rho_{1}\right)\left(\rho_{1}\right)^{\frac{\rho_{1}}{1-\rho_{1}}}|\mu|^{\frac{1}{1-\rho_{1}}}\left(r_{2}-r\right)^{\frac{\rho_{1}}{\rho_{1}-1}} ; \mu=\max \left\{\left|\mu_{l}\right|: 0 \leqslant l \leqslant N_{1}\right\}$, $\mu_{l}$ - корни многочлена $P_{1}(y):=\sum_{s \in N_{4}} a_{s, \nu_{s}} y^{s}$. В силу единственности решения з.К. (26), (27) $w_{1}\left(z_{1}, z_{2}\right)=w_{2}\left(z_{1}, z_{2}\right)$. Поэтому $\forall z_{1} \in \mathbb{C} w_{1}\left(z_{1}, z_{2}\right) \in \mathscr{H}\left(K_{r_{2}}\right)$ и $\forall z_{2} \in K_{r_{2}} w_{1}\left(z_{1}, z_{2}\right) \in\left[\frac{1}{1-\rho_{1}}, \sigma\left(\left|z_{2}\right|\right)\right]$. Отсюда следует, что при $l=0,1,2, \ldots$ $\forall z_{2} \in K_{r_{2}} \frac{\partial^{l} w_{1}\left(z_{1}, z_{2}\right)}{\partial z_{2}^{l}} \in\left[\frac{1}{1-\rho_{1}}, \sigma\left(\left|z_{2}\right|\right)\right]$ и, в частности,

$$
\left.\frac{\partial^{l} w_{1}\left(z_{1}, z_{2}\right)}{\partial z_{2}^{l}}\right|_{z_{2}=0} \in\left[\frac{1}{1-\rho_{1}}, \sigma(0)\right]
$$

где $\sigma(0)=\left(1-\rho_{1}\right)\left(\rho_{1}\right)^{\frac{\rho_{1}}{1-\rho_{1}}}|\mu|^{\frac{1}{1-\rho_{1}}} r_{2}^{\frac{\rho_{1}}{\rho_{1}-1}}$.

Мы получили такое частичное обрашение первого следствия теоремы 10:

ТЕОРема 18. Пусть $\rho_{2}>1, \rho_{1}<1, \nu_{p}=0$. Предположим, что начальные функиии $\varphi_{j}\left(z_{1}\right), 0<j \leqslant m-1$, аналитичны в некотором круге $\left|z_{1}\right|<r_{1}$ и что существует решение $w\left(z_{1}, z_{2}\right)$ з.K. (1), (2) из $\mathscr{H}\left(K_{r_{1}} \times K_{r_{2}}\right)$ при некотором $r_{2}>0$. Тогда при $j=0,1, \ldots, m-1 \quad \varphi_{j}\left(z_{1}\right) \in\left[\frac{1}{1-\rho_{1}}, \sigma\right]$, где $\sigma=\sigma(0)$.

Непосредственно из теоремы 18 и следствия теоремы 10 получаем

СЛЕДСТВИЕ 1. Пусть $\rho_{2}>1, \rho_{1}=1 / \rho_{2}, \nu_{p}=0$. Тогда:

1) если начальные функиии $\varphi_{j}\left(z_{1}\right)$ аналитичны в $\left|z_{1}\right|<r$ и существует решение з.K. (1), (2) из $\mathscr{H}\left(K_{r_{1}} \times K_{r_{2}}\right)$, то $\varphi_{j}\left(z_{1}\right) \in\left[\frac{\rho_{2}}{\rho_{2}-1}, \sigma\right]$, где $\sigma=$ $\left(\rho_{2}-1\right)\left(\rho_{2}\right)^{\frac{\rho_{2}}{1-\rho_{2}}}|\mu|^{\frac{\rho_{2}}{\rho_{2}-1}} r_{2}^{\frac{1}{1-\rho_{2}}}$ u $\mu$-наибольший из модулей корней уравнения $\sum_{s \in \mathscr{N}_{4}} a_{s, \nu_{s}} y^{s}=0$

2) если $\varphi_{j} \in\left[\frac{\rho_{2}}{\rho_{2}-1}, \lambda\right], \quad 0<\lambda<\infty, \quad j=0,1, \ldots, m-1$, то существует решение з.K. (1), (2) из

$$
\mathscr{E}\left[\frac{\rho_{2}}{\rho_{2}-1}, \sigma ; \eta, \tau\right] \subseteq\left(\left[\frac{\rho_{2}}{\rho_{2}-1}, \lambda\right] ; \mathscr{H}\left(K_{\eta / \tau}\right)\right),
$$

где $\eta=\left(\frac{\rho_{2}-1}{\rho_{2}}\right)^{\rho_{2}-1} \frac{\lambda^{1-\rho_{2}}}{\rho_{2}} u \tau-$ наибольший из модулей корней уравнения $\sum_{k \in \mathcal{N}_{2}} a_{l_{k}, k} x^{k}=0$.

Предположим, что все корни последнего уравнения имеют одинаковую абсолютную величину $\tau$. Тогда из сказанного в $\S 7$ (перед теоремой 17 ) следует, что в этом случае $\mu=\tau^{-1 / \rho_{2}}$. В этом случае следствие 1 дает точньй результат:

СлЕДСТВИЕ 2. Пусть $\rho_{2}>1, \rho_{1}=1 / \rho_{2}, \quad \nu_{p}=0 u \mu=\tau^{-1 / \rho_{2}}$. Пусть , далее, начальнье функиии $\varphi_{j}(0 \leqslant j \leqslant m-1)$ аналитичны в начале координат. Тогда следующие утверждения равносильны:

1) $\exists \sigma \in(0,+\infty): \varphi_{j} \in\left[\frac{\rho_{2}}{\rho_{2}-1}, \sigma\right], j=0,1, \ldots, m-1$;

2) существует решение з.K. (1), (2) из $\mathscr{H}\left(\mathbb{C} \times K_{R}\right)$, әде

$$
\tau R\left(\rho_{2}\right)^{\rho_{2}} \cdot(\sigma)^{\rho_{2}-1}=\left(\rho_{2}-1\right)^{\rho_{2}-1}
$$

3) существует решение з.K. (1), (2) из $\mathscr{E}\left[\frac{\rho_{2}}{\rho_{2}-1}, \sigma ; R \tau, \sigma\right]$, где число $R$ определяется из равенства (29); 
4) при некотором $R_{1}>0$ существует решение з.K. (1), (2) из пространства $\mathscr{H}\left(K_{R_{1}} \times K_{R}\right)$.

Еще один точный результат получается, если предположить, что начальные функции $\varphi_{j}, 0 \leqslant j \leqslant m-1$, целые. Тогда, выбирая в следствии 1 теоремы 10 число $\sigma$ равным нулю, а в теореме 18 взяв $\tau_{2}$ как угодно большим, приходим к такому результату:

ТЕОРема 19. Пусть выполнены условия (25), и пусть $\varphi_{j} \in \mathscr{H}(\mathbb{C}), \quad 0 \leqslant j \leqslant$ m-1. Тогда следующие утверждения равносильны:

1) $\varphi_{j} \in\left[\frac{\rho_{2}}{\rho_{2}-1}, 0\right], j=0,1, \ldots, m-1$;

2) существует решение з.К. (1), (2) из $\mathscr{H}\left(\mathbb{C}^{2}\right)$;

3) существует решение з.К. $(1),(2)$ из $\left(\mathscr{H}\left(\mathbb{C}^{2}\right) ;\left[\frac{\rho_{2}}{\rho_{2}-1}, 0\right]\right)$, представимое в виде ряда (5), который строится по ряду (6) и сходится абсолютно в пространстве Фреше $\mathscr{E}[\nu, 0 ; \infty]$ всех иелых функиий $y\left(z_{1}, z_{2}\right)$, для которых $\forall n \geqslant 1$

$$
\|y\|_{n}:=\sup \left\{\frac{\left|y\left(z_{1}, z_{2}\right)\right|}{\exp \left(\left|z_{1}\right|^{\nu} / n\right)}\left|z_{1} \in \mathbb{C},\right| z_{2} \mid \leqslant n\right\}<\infty, \quad \nu=\frac{\rho_{2}}{\rho_{2}-1} .
$$

Наконец, из следствия 1 без труда получаем еще один точный результат.

ТЕОРЕМа 20. Пусть выполнены условия (25), и пусть $\varphi_{j}$ аналитичны в некотором круге $\left|z_{1}\right|<r_{1}$. Тогда следуюшие утвержсдения равносильнь:

1) $\varphi_{j} \in\left[\frac{\rho_{2}}{\rho_{2}-1}, \infty\right), j=0,1, \ldots, m-1$;

2) существует решение з.K. (1), (2), аналитическое в бикруге $K_{r_{1}} \times K_{r_{2}}$ при некотором $r_{2}>0$.

СЛЕДСТВИЕ. Пусть выполнены условия (25), и пусть начальные функиии

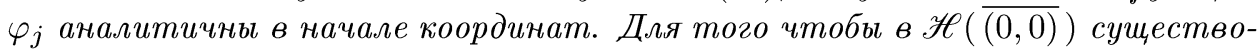
вало решение з.К. (1), (2), необходимо и достаточно, чтобы функиии $\varphi_{j}$ были иельми и имели конечный тип при порядке $\frac{\rho_{2}}{\rho_{2}-1}$.

Мы довольно подробно рассмотрели вопрос об обрашении следствия 1 теоремы 10. Тем же методом можно получить аналогичные результаты для первого следствия теоремы 11.

ТЕОрема 21. Пусть $1<\delta<\frac{\rho_{2}}{\rho_{2}-1}$, и пусть выполнены условия (25). Тогда следуюшие утвержсдения равносильны:

1) $\varphi_{j}\left(z_{1}\right) \in[\delta, 0], j=0,1, \ldots, m-1$;

2) существует решение з.К. (1), (2) из $\mathscr{H}\left(\mathbb{C}^{2}\right)$;

3) существует решение з.К. (1), (2), представимое в виде ряда (5), который сходится абсолютно в $\mathscr{E}_{0}\left[\frac{\delta}{\delta-\delta \rho_{2}+\rho_{2}}, \delta\right]$.

Рассмотрим в качестве примера уравнение Соболева-Гальперна:

$$
\sum_{k=0}^{l_{1}} a_{k} \frac{\partial^{k+1} u\left(x_{1}, x_{2}\right)}{\partial x_{1}^{k} \partial x_{2}}=\sum_{s=0}^{l_{0}} b_{s} \frac{\partial^{s} u\left(x_{1}, x_{2}\right)}{\partial x_{1}^{s}}
$$

где $a_{l_{1}} \cdot b_{l_{0}} \neq 0$. Как легко подсчитать, в данном случае $m=1, l_{m}=l_{1}, \rho_{2}=l_{0}-l_{1}$ и кор. инд. $\rho_{2}-$ целое число. Если $\rho_{2} \leqslant 0$, т.е. если $l_{0} \leqslant l_{1}$, то применима 
теорема 1 из $\S 4$. Далее, если $\rho_{2}>0$, то $\rho_{2} \geqslant 1$. При $\rho_{2}=1$, т.е. при $l_{0}=l_{1}+1$, применимы все результаты из $\S 6$, а именно теоремы 6-9. Пусть, наконец, $\rho_{2}>1$, т.е. $l_{0} \geqslant l_{1}+2$. Легко проверить, что в этом случае в обозначениях $\S 8 p=l_{0}$, $\nu_{p}=0, \rho_{1}=\frac{1}{l_{0}-l_{1}}$, причем величина $\rho_{1}=\max \left\{\frac{\nu_{s}}{l_{0}-s}: 0 \leqslant s<l_{0}, a_{s, \nu_{s}} \neq 0\right\}$ достигается только при $s=l_{1}$. Для рассматриваемого уравнения $\rho_{2} \rho_{1}=1$, а условие 2) теоремы 17 принимает вид $l_{0}>l_{1}+1$, т.е. всегда выполняется при $\rho_{2}>1$. Далее, $P(x)=a_{l_{1}} x+b_{l_{0}}, P_{1}(y)=b_{l_{0}} y^{l_{0}}+a_{l_{1}} y^{l_{1}}$. Так как теорема 17 в данном случае применима, то для уравнения Соболева-Гальперна справедливы все результаты не только $\S 7$, но и данного параграфа.

В заключение отметим, что в случае $\rho_{2}>1$ обращение всех следствий "прямых" теорем 10, 11 удалось осушествить, в отличие от ранее разобранных случаев, в которых $\rho_{2} \leqslant 1$, только при определенных дополнительных предположениях. При этом остались нерешенными такие, на наш взгляд, довольно интересные задачи.

1) Когда $\rho_{1} \neq \frac{1}{\rho_{2}}, \rho_{1}<1$, но $\nu_{p}=0$ (т.е. единственность з.К. по $z_{1}$ имеется), дать точное описание пространства $E$ целых функций такого, что в случае, если начальные функции $\varphi_{j}$ принадлежат $E$ (и только в этом случае), существует решение з.К. (1), (2) из $\mathscr{H}\left(K_{r_{1}} \times K_{r_{2}}\right)$ при некоторых $r_{1}, r_{2}>0$.

Из теорем 10 и 18 следует, что $\left[\frac{\rho_{2}}{\rho_{2}-1}, \infty\right) \subset E \subset\left[\frac{1}{1-\rho_{1}}, \infty\right)$.

2) Дать хотя бы частичное обращение теорем 10,11 в случае, когда $\rho_{2}>1$, $\nu_{p}=0$, но $\rho_{1} \geqslant 1$.

Примененный в настоящем параграфе метод основан на предположении единственности решения в рассматриваемых классах вспомогательной з.К. (23), (26) по переменной $z_{1}$, т.е. на предположении, что $\nu_{p}=0$. Было бы весьма интересно, привлекая другие методы, получить обращение первых следствий теорем 10, 11, отказавшись от предположения $\nu_{p}=0$.

\section{§ 10. Свойства пространства начальных данных}

Рассмотрим уравнение (1) в пространстве $A_{0,2}:=\mathscr{H}(\overline{(0,0)})=\operatorname{ind}_{n} \mathscr{H}\left(K_{r_{n}} \times\right.$ $\left.K_{r_{n}}\right) \quad\left(r_{n} \downarrow 0\right)$ аналитических ростков в начале координат. Пусть еше $\overline{A_{0}}=$ ind $\mathscr{H}\left(K_{r_{n}}\right)$. Как известно (см., например, [18]), $\overline{A_{0}}$ и $A_{0,2}-L N^{*}$-пространства. Определим оператор $T$ :

$$
\forall w \in A_{0,2} \rightarrow T w=\left\{w\left(0, z_{1}\right),\left.\frac{\partial w}{\partial z_{2}}\right|_{z_{2}=0}, \ldots,\left.\frac{\partial^{m-1} w}{\partial z_{2}^{m-1}}\right|_{z_{2}=0}\right\} \in \overline{A_{0}^{m}}
$$

Тогда $T$ - линейный непрерывный оператор из $A_{0,2}$ в $\overline{A_{0}^{m}}$. Пусть $E:=T\left(A_{0,2}\right)$ - образ оператора $T$. Из полученных в $\S 4-6$ результатов следует, в частности, что при $\rho_{2} \leqslant 1 E=\overline{A_{0}^{m}}$. В настояшем параграфе мы опишем некоторые топологические свойства множества $E$ в случае, когда $\rho_{2}>1$. Зададим в векторном пространстве $E$ топологию $\lambda$, индуцированную из $\overline{A_{0}^{m}}$. Назовем з.К. (1), (2) $\lambda$-возвратно-ограниченной, если для любого подмножества $Q$ пространства $E$, ограниченного в топологии $\lambda$, множество $T^{-1}(Q)$ ограничено в $A_{0,2}$.

Покажем, что если $\rho_{2}>1$, то з.К. (1), (2) не будет $\lambda$-возвратно-ограниченной. Допустим, что это не так. Положим, как выше, $\rho_{0}=\frac{\rho_{2}}{\rho_{2}-1}$ и выберем при каком-либо $R \in(0,+\infty) \mathrm{A \Pi C} \mathscr{E}_{\Lambda}:=\left(\exp \lambda_{k} z_{1}\right)_{k=1}^{\infty}$ в $\mathscr{H}\left(K_{R}\right)$ так, чтобы $\lim \sup _{k} \frac{k}{\left|\lambda_{k}\right|}<\infty$. Возьмем любую функцию $v$ из $\mathscr{H}\left(K_{R}\right) \backslash\left[\rho_{0}, \infty\right)$, т.е. любую 
аналитическую в круге $\left|z_{1}\right|<R$ функцию,не являющуюся целой функцией конечного типа при порядке $\rho_{0}$. Пусть

$$
w\left(z_{1}\right)=\sum_{k=1}^{\infty} c_{k} \exp \lambda_{k} z_{1}
$$

- какое-либо ее разложение в абсолютно сходящийся в $\mathscr{H}\left(K_{R}\right)$ ряд по АПС $\mathscr{E}_{\Lambda}$. Этот ряд сходится абсолютно в $\overline{A_{0}}$, и поэтому множество $\left\{c_{k} \exp \lambda_{k} z_{1}: k=\right.$ $1,2, \ldots\}$ ограничено в $\overline{A_{0}}$.

Пусть $\mu(\lambda)$ - ветвь, определяемая уравнением $Q(\lambda, \mu)=0$. Тогда $\lim _{\lambda \rightarrow \infty} \mu(\lambda) \lambda^{-\rho_{2}}=\tau$, где $\tau-$ один из корней многочлена $P(x)$. Выберем этот корень так, чтобы $\tau \neq 0$, и рассмотрим в $\overline{A_{0}^{m}}$ семейство $\Phi=\left\{\mathscr{F}_{k}: k=1,2, \ldots\right\}$, где $\forall k \geqslant 1$

$$
\mathscr{F}_{k}=\left(c_{k} \exp \lambda_{k} z_{1}, c_{k} \mu_{k} \exp \lambda_{k} z_{1}, \ldots, c_{k} \mu_{k}^{m-1} \exp \lambda_{k} z_{1}\right), \quad \mu_{k}=\mu\left(\lambda_{k}\right) .
$$

Так как оператор дифференцирования непрерывен в $\overline{A_{0}}$ и $\exists D<\infty:\left|\mu_{k}\right| \leqslant D\left|\lambda_{k}\right|^{\rho_{2}}$, $k=1,2, \ldots$, то $\Phi-$ ограниченное множество в $\overline{A_{0}^{m}}$. Рассмотрим функцию

$$
w_{k}\left(z_{1}, z_{2}\right)=c_{k} \exp \left(\lambda_{k} z_{1}+\mu_{k} z_{2}\right), \quad k=1,2, \ldots
$$

Очевидно, что $\forall k \geqslant 1 T w_{k}=\mathscr{F}_{k}$. Пусть $\gamma=\left(w_{k}: k=1,2, \ldots\right)$. Тогда $\gamma \subseteq$ $T^{-1}(\Phi)$ и по допущению $\gamma$ ограничено в $A_{0,2}$. Но так как $A_{0,2}-\mathrm{LN}^{*}$-пространство, то оно подавно будет регулярным индуктивным пределом:

$$
\begin{gathered}
\exists r>0, \quad \exists M<\infty: \forall k \geqslant 1 \\
\left|c_{k}\right| \max \left\{\left|\exp \left(\lambda_{k} z_{1}+\mu_{k} z_{2}\right)\right|:\left|z_{1}\right| \leqslant r,\left|z_{2}\right| \leqslant r\right\} \leqslant M,
\end{gathered}
$$

т.е. $\left|c_{k}\right| \exp \left(\left|\lambda_{k}\right|+\left|\mu_{k}\right|\right) r \leqslant M, k=1,2, \ldots$, и подавно $\forall k \geqslant 1\left|c_{k}\right| \exp \left|\mu_{k}\right| r \leqslant M$. Отсюда $\lim \sup _{k}\left(\left|\mu_{k}\right|^{-1} \ln \left|c_{k}\right|+r\right) \leqslant 0$, т.е. $\limsup _{k}\left(\left|\lambda_{k}\right|^{-\rho_{2}} \ln \left|c_{k}\right|+\tau r\right) \leqslant 0$. Но тогда ряд $\sum_{k=1}^{\infty} c_{k} \exp \lambda_{k} z_{1}$ сходится абсолютно в $\left[\rho_{0}, \sigma_{0}\right]$, где, как выше, $\rho_{0}=$ $\frac{\rho_{2}}{\rho_{2}-1}$, а $\sigma_{0}$ определяется из уравнения $\left(\rho_{0} \sigma_{0}\right)^{1 / \rho_{0}} \cdot\left(\rho_{2} \tau r\right)^{1 / \rho_{2}}=1$. Его сумма, совпадающая с $v\left(z_{1}\right)$ в некоторой окрестности начала координат, принадлежит пространству целых функций $\left[\rho_{0}, \sigma_{0}\right] \subset\left[\rho_{0}, \infty\right)$, что невозможно.

Применим полученный результат к уравнению (1) $\mathrm{c} l_{m}=0$ :

$$
\frac{\partial^{m} w\left(z_{1}, z_{2}\right)}{\partial z_{2}^{m}}+\sum_{k=0}^{m-1} \sum_{j=0}^{l_{k}} a_{j, k} \frac{\partial^{j+k} w\left(z_{1}, z_{2}\right)}{\partial z_{1}^{j} \partial z_{2}^{k}}=0
$$

ТЕОРема 22. Если для уравнения (30) $\rho_{2}>1$, то множество Е незамкнуmo $в \overline{A_{0}^{m}}$.

ДоказАТЕльство. Допустим, рассуждая от противного, что $E$ замкнуто. Тогда $E$ в индуцированной из $\overline{A_{0}^{m}}$ топологии является, как известно, LN*-пространством. Линейный оператор $T$ отображает непрерывно и инъективно LN*-пространство $A_{0,2}$ на такое же пространство $E$. По теореме Гротендика $T$ - изоморфизм. Но тогда з.К. (30), (2) $\lambda_{0}$-возвратно-ограничена, что невозможно. 
СЛЕДСТВИЕ 1. Для того чтобы кор. инд. $\rho_{2}$ уравнения (1) был больше единиць, достаточно, а в случае, если $l_{m}=0$, и необходимо, чтобь $E=$ $T(\mathscr{H}\{\overline{(0,0)}\})$ было собственным незамкнутылм подпространством $\overline{A_{0}^{m}}$.

СлЕДСТВИЕ 2. Для уравнения (30) равносильны такие утверждения:

1) $\rho_{2}>1$

2) образ оператора $T$ незамкнут в $\overline{A_{0}^{m}}$;

3) оператор $T: A_{0,2} \rightarrow \overline{A_{0}^{m}}$ не сюргективен;

4) $T\left(A_{0,2}\right)$ - незамкнутое собственное подмножество в $\overline{A_{0}^{m}}$.

\section{$\S 11$. О линейной непрерывной зависимости решения задачи Коши от начальных данных}

Для того чтобы цели настоящего параграфа и методы их достижения были более прозрачными, дадим постановку задачи и приведем полученные в процессе ее решения результаты в одной довольно типичной для данной работы ситуации, которая уже встречалась в $\S 6$. Пусть $\rho_{2}=1$ и $\mathscr{G}_{0}$ - выпуклая область в $\mathbb{C}$. Обозначим через $T$ линейный оператор, ставящий в соответствие каждой функции $w\left(z_{1}, z_{2}\right)$ из $\mathscr{H}\left(\mathscr{P} \mathscr{G}_{0}\right)$ систему $m$ функций от $z_{1}$ :

$$
w\left(z_{1}, 0\right),\left.\frac{\partial w\left(z_{1}, z_{2}\right)}{\partial z_{2}}\right|_{z_{2}=0}, \ldots,\left.\frac{\partial^{m-1} w\left(z_{1}, z_{2}\right)}{\partial z_{2}^{m-1}}\right|_{z_{2}=0},
$$

т.е. элемент пространства $\mathscr{H}\left(\mathscr{G}_{0}\right)^{m}:=\mathscr{H}\left(\mathscr{G}_{0}\right) \times \cdots \times \mathscr{H}\left(\mathscr{G}_{0}\right)$. Если в $\mathscr{H}\left(\mathscr{P} \mathscr{G}_{0}\right)$ и в $\mathscr{H}\left(\mathscr{G}_{0}\right)^{m}$ ввести стандартные топологии, то $T$ - линейный непрерывный оператор из $\mathscr{H}\left(\mathscr{P} \mathscr{G}_{0}\right)$ в $\mathscr{H}\left(\mathscr{G}_{0}\right)^{m}$.

Обратимся теперь к оператору $M$, стоящему в левой части уравнения (1). Очевидно, что $M-$ линейный непрерывный оператор в $\mathscr{H}\left(\mathscr{P} \mathscr{G}_{0}\right)$. Тогда его ядро является замкнутым подпространством пространства $\Phi$ реше $\mathscr{H}\left(\mathscr{P} \mathscr{G}_{0}\right)$, т.е. также пространством Фреше (в индуцированной из $\mathscr{H}\left(\mathscr{P} \mathscr{G}_{0}\right)$ топологии). Оператор $T$ непрерывно действует из $M^{-1}(0)$ в $\mathscr{H}\left(\mathscr{G}_{0}\right)^{m}$ и по теореме 6 является эпиморфизмом. Согласно известной теореме Майкла $[20] T$ имеет непрерывный правый обратный оператор $Q: M^{-1}(0) \rightarrow \mathscr{H}\left(\mathscr{G}_{0}\right)^{m}$,

$$
\forall \varphi\left(\varphi_{0}, \varphi_{1}, \ldots, \varphi_{m-1}\right) \in\left(\mathscr{H}\left(\mathscr{G}_{0}\right)\right)^{m} \quad T Q \varphi=\varphi .
$$

Функция $Q \varphi$ из $M^{-1}(0)$ зависит непрерывно от начальных данных $\varphi$. Следовательно, в предположениях теоремы 6 всегда существует решение з.К. (1), (2), непрерывно зависящее от начальных данных. Однако оператор $Q$ в общей ситуации нелинеен. В то же время в ряде случаев важно знать, что имеется решение з.К. (1), (2), зависящее от начальных данных не только непрерьвно, но и линейно. Иными словами, важно знать, когда оператор $T$ (точнее, его сужение $T_{M}$ на $\left.M^{-1}(0)\right)$ имеет линейньй непрерывный правый обратный (ЛНПО) $Q$.

Можно описать один простой случай, когда такой оператор $Q$ имеется. Именно, если нулевая з.К. для уравнения (1) по $z_{2}$ :

$$
\left.\frac{\partial^{s} w\left(z_{1}, z_{2}\right)}{\partial z_{2}^{s}}\right|_{z_{2}=0}=0, \quad s=0,1, \ldots, m-1,
$$


имеет только тривиальное решение в $\mathscr{H}\left(\mathscr{P} \mathscr{G}_{0}\right)$, то оператор $T_{M}: M^{-1}(0) \rightarrow$ $\left(\mathscr{H}\left(\mathscr{G}_{0}\right)\right)^{m}$ инъективен и сюръективен, и по теореме Банаха $T_{M}$ - изоморфизм.

Если же единственность решения з.К. (1), (31) в $\mathscr{H}\left(\mathscr{P} \mathscr{G}_{0}\right)$ не гарантируется, то вопрос о сушествовании ЛНПО у $T_{M}$ становится довольно сложным. Согласно $[21$, $\S 1$, п. 8] для существования такого оператора необходимо и достаточно, чтобы ядро $T_{M}^{-1}(0)$ оператора $T_{M}$, т.е. множество всех решений нулевой з.К. (1), (31) было дополнимо в $M^{-1}(0)$ - во множестве всех решений уравнения (1) из $\mathscr{H}\left(\mathscr{P} \mathscr{G}_{0}\right)$. Однако вопрос о дополнимости $T_{M}^{-1}(0)$ в $M^{-1}(0)$ тоже весьма сложен, и его решение должно быть объектом самостоятельного исследования.

Оставаясь в рамках методов, используемых в настоящей статье, мы покажем, что привлечение АПС экспонент дает возможность указать как условия достаточно обшего характера для существования ЛНПО у $T_{M}$, так и способ его построения. Для этого нам понадобится оператор представления $L$, играющий важную роль в теории АПС (см., например, [5]). В рассматриваемой ситуации этот оператор определяется следующим образом. Пусть $\mathscr{E}_{\Lambda}=\left\{\exp \lambda_{k} z\right\}_{k=1}^{\infty}-$ какая-либо последовательность экспонент в $\mathscr{H}\left(\mathscr{G}_{0}\right)$ и $\left(\mathscr{G}_{n}\right)_{n=1}^{\infty}$ - возрастающая последовательность выпуклых компактов, аппроксимирующих изнутри область $\mathscr{G}_{0}$. Топология в $\mathscr{H}\left(\mathscr{G}_{0}\right)$ задается счетным набором преднорм $p_{n}(y)=\max \left\{|y(z)|: z \in \mathscr{G}_{n}\right\}$, $n=1,2, \ldots$ Обозначим символом $A_{2}\left(\mathscr{E}_{\Lambda}\right)$ пространство всех числовых последовательностей $c=\left(c_{k}\right)_{k=1}^{\infty}$ таких, что

$$
q_{n}(c):=\sum_{k=1}^{\infty}\left|c_{k}\right| p_{n}\left(\exp \lambda_{k} z_{1}\right)<\infty, \quad n=1,2, \ldots
$$

Пространство $A_{2}\left(\mathscr{E}_{\Lambda}\right)$ с набором преднорм $\left(q_{n}(c)\right)_{k=1}^{\infty}$ является пространством $\Phi$ реше (см. [5]). Оператор представления $L$ ставит в соответствие каждой последовательности $c=\left(c_{k}\right)_{k=1}^{\infty}$ из $A_{2}\left(\mathscr{E}_{\Lambda}\right)$ функцию $L c=\sum_{k=1}^{\infty} c_{k} \exp \lambda_{k} z_{1}$ из $\mathscr{H}\left(\mathscr{G}_{0}\right)$. Очевидно, что $L$ непрерывен и что $L$ является эпиморфизмом тогда и только тогда, когда $\mathscr{E}_{\Lambda}-\mathrm{A \Pi C}$ в $\mathscr{H}\left(\mathscr{G}_{0}\right)$. Будем далее предполагать, что $\mathscr{E}_{\Lambda}-\mathrm{A} \Pi \mathrm{\text {в }} \mathscr{H}\left(\mathscr{G}_{0}\right)$. Тогда $L$ - эпиморфизм $A_{2}\left(\mathscr{E}_{\Lambda}\right)$ на $\mathscr{H}\left(\mathscr{G}_{0}\right)$. Допустим, что он имеет ЛНПО $B: \mathscr{H}\left(\mathscr{G}_{0}\right) \rightarrow$ $A_{2}\left(\mathscr{E}_{\Lambda}\right)$. Тогда любой набор начальных функций $\left(\varphi_{j}\left(z_{1}\right)\right)_{0}^{m-1}$ из $\left(\mathscr{H}\left(\mathscr{G}_{0}\right)\right)^{m}$ можно представить в виде абсолютно сходяшихся в $\mathscr{H}\left(\mathscr{G}_{0}\right)$ рядов:

$$
\varphi_{j}\left(z_{1}\right)=\sum_{k=1}^{\infty}\left(B \varphi_{j}\right)_{k} \exp \lambda_{k} z_{1}, \quad j=0, \ldots, m-1
$$

где $B \varphi=\left\{(B \varphi)_{k}\right\}_{k=1}^{\infty} \forall \varphi \in \mathscr{H}\left(\mathscr{G}_{0}\right)$.

Рассмотрим систему (7), положив в ней $b_{k, n}=\left(B \varphi_{n}\right)_{k}, n=0,1, \ldots, m-1$, $k=1,2, \ldots$ Из этой системы определяем единственным образом (при любом фиксированном $k \geqslant 1$ ) величины $a_{j, s}^{(k)}$ по формулам Крамера:

$$
a_{j, s}^{(k)}=A_{j, s, k}\left(F_{k}\right)
$$

где

$$
F_{k}=\left(\left(B \varphi_{0}\right)_{k},\left(B \varphi_{1}\right)_{k}, \ldots,\left(B \varphi_{m-1}\right)_{k}\right) \in \mathbb{C}^{m}
$$


и $A_{j, s, k}-$ линейный оператор в $\mathbb{C}^{m}$. Подставляя эти значения $a_{j, s}^{(k)}$ в $(5)$, получим

$$
w_{0}\left(z_{1}, z_{2}\right)=\sum_{j=1}^{N} \sum_{s=0}^{p_{j}-1} \sum_{k=1}^{\infty} A_{j, s, k}\left(F_{k}\right) z_{2}^{s} \exp \left[\lambda_{k} z_{1}+\mu_{j}\left(\lambda_{k}\right) z_{2}\right]
$$

Очевидно, что правая часть равенства (32) зависит от начальных функций $\left(\varphi_{j}\right)_{j=0}^{m-1}$ линейно. Покажем, что эта зависимость и непрерьвна. Пусть $\varphi\left(\varphi_{0}, \ldots\right.$ $\left.\ldots, \varphi_{m-1}\right)$ - любой элемент из $\left(\mathscr{H}\left(\mathscr{G}_{0}\right)\right)^{m}$, и пусть $\mathscr{F}-$ произвольный компакт в $\mathscr{P} \mathscr{G}_{0}$. Тогда $\mathscr{F}_{1}:=\bigcup_{j=1}^{m}\left\{z_{1}+\tau_{j} z_{2}:\left(z_{1}, z_{2}\right) \in \mathscr{F}\right\}-$ компакт в $\mathscr{G}_{0}$ и найдется номер $K \geqslant 1$ такой, что $\mathscr{F}_{1} \subset \mathscr{G}_{K+1}$. Проведя те же оценки, что и в $\S 5$, и учитывая, как и там, неравенства $(9)$, приходим к оценке $(14)$, в которой $b_{k, n}$ заменено на $\left(B \varphi_{n}\right)_{k}$ : при $k \geqslant k_{0}$

$$
\begin{aligned}
\gamma_{j, s}^{(k)} & \leqslant D_{1} \sum_{n=0}^{m-1}\left|\left(B \varphi_{n}\right)_{k}\right| \exp \max \left\{\operatorname{Re} \lambda_{k} v: v \in \mathscr{G}_{K+1}\right\} \\
& =D_{1} \sum_{n=0}^{m-1}\left|\left(B \varphi_{n}\right)_{k}\right| p_{K+1}\left(\exp \lambda_{k} z\right)
\end{aligned}
$$

для всех $1 \leqslant j \leqslant N, 0 \leqslant s \leqslant p_{j}-1$. Увеличив, в случае необходимости, константу $D_{1}$, можно добиться того, чтобы для тех же $j, s$ и для всех $l \geqslant 1$ и любого $\varphi$ из $\left(\mathscr{H}\left(\mathscr{G}_{0}\right)\right)^{m}$

$$
\gamma_{j, s}^{(l)} \leqslant D_{1} \sum_{n=0}^{m-1}\left|\left(B \varphi_{n}\right)_{k}\right| p_{K+1}\left(\exp \lambda_{l} z\right)
$$

Но тогда

$$
\begin{aligned}
& \max \left\{\left|w_{0}\left(z_{1}, z_{2}\right)\right|:\left(z_{1}, z_{2}\right) \in \mathscr{F}\right\} \leqslant \sum_{j=1}^{N} \sum_{s=0}^{p_{j}-1} \sum_{l=1}^{\infty} \gamma_{j, s}^{(l)} \\
& \leqslant D_{1}\left(\sum_{j=1}^{N} p_{j}\right) \sum_{n=0}^{m-1} \sum_{l=1}^{\infty}\left|\left(B \varphi_{n}\right)_{l}\right| p_{K+1}\left(\exp \lambda_{l} z\right)=D_{2} \sum_{n=0}^{m-1} q_{K+1}\left(B \varphi_{n}\right) .
\end{aligned}
$$

Выберем по номеру $K$ номер $K_{1}$ и постоянную $D_{3}$ такие, что $\forall f \in \mathscr{H}\left(\mathscr{G}_{0}\right)$ $q_{K+1}(B f) \leqslant D_{3} p_{K_{1}}(f)$. В итоге

$$
\max \left\{\left|w_{0}\left(z_{1}, z_{2}\right)\right|:\left(z_{1}, z_{2}\right) \in \mathscr{F}\right\} \leqslant D_{1} D_{3} \sum_{n=0}^{m-1} p_{K_{1}}\left(\varphi_{n}\right)
$$

Таким образом, правая часть равенства (32) является линейным непрерьвным оператором из $\left(\mathscr{H}\left(\mathscr{G}_{0}\right)\right)^{m}$ в $\mathscr{H}\left(\mathscr{P} \mathscr{G}_{0}\right)$, причем

$$
T_{M} w_{0}=\varphi:=\left(\varphi_{0}, \varphi_{1}, \ldots, \varphi_{m-1}\right)
$$

Следовательно, $T_{M}$ имеет ЛНПО, представимый в виде (32). 
Нам осталось указать условия, при которых оператор представления $L$ имеет ЛНПО. Пусть $\psi(z)$ - какая-либо функция, конформно отображающая единичньй круг $|z|<1$ на область $\mathscr{G}_{0}$. Согласно [22] если область $\mathscr{G}_{0}$ ограничена и $\sup _{|z|<1}\left|\psi^{\prime}(z)\right|<\infty$, то найдется АПС $\mathscr{E}_{\Lambda}$ в $\mathscr{H}\left(\mathscr{G}_{0}\right)$ такая, что $\lim \sup _{k} \frac{k}{\left|\lambda_{k}\right|}<\infty$ и соответствующий оператор представления $L$ имеет ЛНПО.

Мы установили такой результат:

ТЕОРема 23. Пусть для уравнения (1) $\rho_{2}=1$. Пусть, далее, $\mathscr{G}_{0}-$ выпуклая область в $\mathbb{C} и \psi(z)$ - функиия, конформно отображающая единичный круг на $\mathscr{G}_{0}$. Тогда:

1) оператор $T_{M}$ имеет непрерывный правый обратный (и, таким образом, существует решение з.К. (1), (2), непрерывно зависящее от начальных данных);

2) если область $\mathscr{G}_{0}$ ограничена и $\sup _{|z|<1}\left|\psi^{\prime}(z)\right|<\infty$, то оператор $T_{M}$ имеет линейный непрерывный правый обратный (и, таким образом, существует решение з.К. (1), (2), зависящее от начальных данных и непрерывно, и линейно).

В частности, $T_{M}$ имеет ЛНПО, если граница ограниченной выпуклой области $\mathscr{G}_{0}$ принадлежит классу $C^{(2)}$.

Теорема 23 дает достаточные условия для существования ЛНПО у оператора $T_{M}$. Было бы интересно, на наш взгляд, получить критерий сушествования ЛНПО.

Использованные в настоящем параграфе соображения применимы и в других ситуациях - например, для пространств $[\rho, \sigma],[\rho, \sigma)$, для случаев $\rho_{2}<1$ и $\rho_{2}>1$ и т.д. Важно лишь, чтобы в пространстве $\mathscr{E}_{1}\left(\mathscr{F}_{1}\right)$, к которому принадлежат начальные функции, имелась АПС экспонент такая, что соответствующий оператор представления обладает ЛНПО. В этом случае точно так же, как в данном параграфе, можно указать достаточные условия существования ЛНПО у оператора $T_{M}$.

\section{§ 12. Упрощение представления решения для невырожденного случая}

Положим при любом фиксированном $\lambda \in \mathbb{C}$

$$
T_{\lambda}(\mu)=Q(\lambda, \mu)=\sum_{k=0}^{m} \mu^{k} \mathscr{R}_{k}(\lambda) .
$$

Образуем дискриминант многочлена (от $\mu) T_{\lambda}(\mu)$. Ясно, что он будет некоторым многочленом $v(\lambda)$ от $\lambda$. Предположим, что многочлен $v(\lambda)$ отличен от тождественного нуля. Тогда $\exists R_{1} \in(0,+\infty): v(\lambda) \neq 0$, если $|\lambda| \geqslant R_{1}$.

Пусть, как раньше $($ см. § 3$), \mathscr{E}_{\Lambda}=\left(\exp \lambda_{k} z_{1}\right)_{k=1}^{\infty}-\mathrm{A \Pi C}$ в $\mathscr{E}_{1}\left(\mathscr{F}_{1}\right)$ и $\lim _{k}\left|\lambda_{k}\right|=\infty$. Пусть, далее, $\mathscr{E}_{1}\left(\mathscr{F}_{1}\right)$ инвариантно относительно дифференцирования, а оператор $y^{\prime}-\alpha y$ при любом $\alpha \in \mathbb{C}$ является эпиморфизмом $\mathscr{E}_{1}\left(\mathscr{F}_{1}\right)$. Тогда по предложению 1 из $\S 3$ последовательность $\left(\exp \lambda_{k} z\right)_{k=N}^{\infty}$ будет АПС в $\mathscr{E}_{1}\left(\mathscr{F}_{1}\right)$ при любом натуральном $N$. Пусть $R_{2} \geqslant R_{1}$ и $R_{2}$ настолько велико, что $\mathscr{R}_{m}(\lambda) \neq 0$, когда $|\lambda| \geqslant R_{2}$. В области $|\lambda|>R_{2}$ уравнение $Q(\lambda, \mu)=0$ имеет точно $m$ простых ветвей $\mu_{j}(\lambda)$, 
$j=1,2, \ldots, m$, соответствующей алгебраической функции. Учитывая предложение 1 , можно всегда считать, что если $\mathscr{E}_{\Lambda}-\mathrm{AПC} \mathrm{в} \mathscr{E}_{1}\left(\mathscr{F}_{1}\right)$, то $\forall k \geqslant 1\left|\lambda_{k}\right| \geqslant R_{2}$. В этом случае решение з.К. (1), (2) можно искать в виде ряда

$$
w_{0}\left(z_{1}, z_{2}\right)=\sum_{j=1}^{m} \sum_{k=1}^{\infty} a_{j}^{(k)} \exp \left[\lambda_{k} z_{1}+\mu_{j}\left(\lambda_{k}\right) z_{2}\right] .
$$

Коэффициенты $a_{j}^{(k)}$ определяются при любом фиксированном $k \geqslant 1$ из следующих соотношений (более простых, чем (7)):

$$
b_{k, n}=\sum_{j=1}^{m} a_{j}^{(k)}\left(\mu_{j, k}\right)^{n}, \quad n=0,1, \ldots, m-1, \quad \mu_{j, k}=\mu_{j}\left(\lambda_{k}\right) .
$$

Определитель системы (34) является определителем Вандермонда и отличен от нуля, так как все числа $\mu_{j, k}, j=1,2, \ldots, m$, попарно различны. Как в $\S 3$, получаем для коэффициентов $a_{j}^{(k)}$, определяемых единственным образом из системы (34), оценку вида (9):

$$
\left|a_{j}^{(k)}\right| \leqslant D\left|\lambda_{k}\right|^{T} \sum_{n=0}^{m-1}\left|b_{k, n}\right|, \quad j=1,2, \ldots, m, \quad k=1,2, \ldots
$$

Все дальнейшие рассуждения, уже изложенные выше, остаются в силе так же, как и результаты, полученные в обеих частях настоящей работы. Единственное различие заключается лишь в том, что решение з.К. имеет представление (33), более простое, чем (5).

Заметим, что если многочлен $Q(\lambda, \mu)$ неприводим, то его дискриминант $T_{\lambda}(\mu)$ всегда отличен от тождественного нуля и, следовательно, решение з.К. (1), (2) можно построить в виде ряда (33).

\section{$\S 13$. Задача Коши в пространстве ростков бесконечно дифференцируемых функций}

В настоящей работе рассматривалась з.К. (1), (2) в различных пространствах обычных функций - бесконечно дифференцируемых или аналитических. Единственным исключением был $\S 10$, в котором исследованы свойства "пространства начальных данных" $E=T\left(A_{0,2}\right)$ для з.К. (1), (2) в пространстве аналитических ростков в точке $(0,0) \in \mathbb{C}^{2}$.

Представляется естественным применить метод настоящей статьи к более общим классам решений з.К. (1), (2) из пространств ростков бесконечно дифференцируемых функций. Уточним прежде всего постановку задачи, дав предварительно несколько определений.

Пусть $Q$ - произвольное непустое подмножество $\mathbb{C}$ и $v(Q)$ - некоторая произвольно зафиксированная совокупность содержащих $Q$ плотных в себе (но не обязательно открытых) множеств $T$ из $\mathbb{C}$ такая, что $\forall T_{j} \in v(Q)(j=1,2) \exists T_{3} \in v(Q)$ : $T_{3} \subseteq T_{1} \cap T_{2}$.

Множества из $v(Q)$ называем окрестностями $Q$ (из системы $v(Q)$ ). 
Пусть $j=1,2$ и $T_{j} \in v(Q)$, где $Q$ - произвольное подмножество $\mathbb{C}$. Две функции $f_{j} \in C^{\infty}\left(T_{j}\right)$ называются әквивалентнылми (по системе $v(Q)$ ), если $\exists T_{3} \in v(Q)$ : $T_{3} \subseteq T_{1} \cap T_{2}$ и $\left.f_{1}\right|_{T_{3}}=\left.f_{2}\right|_{T_{3}}$.

Класс эквивалентности, состояший из всех эквивалентных друг другу функций, назовем $\left(C^{\infty}, v(Q)\right)$-ростком, а векторное пространство, составленное из всех таких ростков, обозначим символом $\left(Q ; C^{\infty} ; v(Q)\right)$.

Приведем некоторые примеры пространств $\left(Q ; C^{\infty} ; v(Q)\right)$, часть которых уже встречалась выше.

1. $Q$ - область в $\mathbb{C} ; v(Q)$ - одноэлементное множество: $v(Q)=\{Q\}$. В данном случае $\left(Q ; C^{\infty} ;\{Q\}\right)$ отождествляется с пространством $A(Q)$ всех аналитических в области $Q$ функций.

2. $Q$ - континуум в $\mathbb{C}$, и $v(Q)$ - множество $D(Q)$ всех областей, содержаших $Q$. Пространство $\left(Q ; C^{\infty} ; D(Q)\right)$ совпадает с пространством $\mathscr{H}(Q)$ всех аналитических ростков на $Q$, рассматривавшимся еще Гротендиком и Мартино.

3. $Q$ - выпуклый компакт в $\mathbb{C}$, и $v(Q)=\{Q\}$. Тогда $\left(Q ; C^{\infty} ;\{Q\}\right)$ совпадает с пространством $A C^{\infty}(Q)$ всех функций, аналитических в Int $Q$ и бесконечно дифференцируемых на $Q$. В частности, если $Q=[-R,+R], 0<R<+\infty$, то $\left(Q ; C^{\infty} ;\{Q\}\right)$ - пространство $C^{\infty}[-R,+R]$ всех функций, определенных и бесконечно дифференцируемых на $[-R,+R]$.

4. Более обшим образом, если $Q$ - любое выпуклое подмножество $\mathbb{R}$ (не обязательно ограниченное), т.е. интервал, сегмент или полуинтервал, а $v(Q)=\{Q\}$, то $\left(Q ; C^{\infty} ;\{Q\}\right)$ совпадает с пространством $C^{\infty}(Q)$ всех бесконечно дифференцируемых в промежутке $Q$ функций.

5. Если $Q=(-R,+R)$, где $0<R<\infty$, и $v(Q)=D(Q)$ - множество всех областей, содержащих $Q$, то $\left(Q ; C^{\infty} ; D(Q)\right)$ - пространство всех аналитических ростков на $Q$, называемое еще пространством вещественно-аналитических функиий наQ.

Будем говорить, что последовательность $\left(g_{n}\right)_{n=1}^{\infty}$ ростков из $\left(Q ; C^{\infty} ; v(Q)\right)$ сходится в пространстве $\left(Q ; C^{\infty} ; v(Q)\right) x$ әлементу $g$ из этого пространства, если $\exists \mathscr{G} \in v(Q): \forall s \geqslant 0$ последовательность $\left(\tilde{g}_{n}^{(s)}\right)_{n=1}^{\infty}$ сходится к $\tilde{g}^{(s)}$ равномерно на каждом компакте из $\mathscr{G}$ (здесь $\tilde{g}_{n}$ и $\tilde{g}$ - представители соответственно ростков $g_{n}$ и $g$, не зависяшие от $s)$.

Пусть $l=1,2, \mathscr{F}_{l} \subseteq \mathbb{C}$ и $v_{l}\left(\mathscr{F}_{l}\right)$ - фиксированная система окрестностей $\mathscr{F}_{l}$. Пусть, далее, $\mathscr{E}_{l}\left(\mathscr{F}_{l}\right)-$ ПОЛВП ростков, вложенное секвенциально непрерывно в $\left(\mathscr{F}_{l} ; C^{\infty} ; v_{l}\left(\mathscr{F}_{l}\right)\right)$.

Обозначим символом $\left(\mathscr{E}_{1}\left(\mathscr{F}_{1}\right) ; \mathscr{E}_{2}\left(\mathscr{F}_{2}\right)\right)$ векторное пространство всех элементов $u\left(z_{1}, z_{2}\right)$ с такими свойствами: при $l=1,2 \exists T_{l} \in v_{l}\left(\mathscr{F}_{l}\right): \forall z_{l} \in T_{l} u\left(z_{1}, z_{2}\right) \in$ $\mathscr{E}_{3-l}\left(\mathscr{F}_{3-l}\right)$.

Предположим, что $0 \in \mathscr{F}_{2}$, и поставим для уравнения (1) задачу Коши по переменной $z_{2}$ : найти решение уравнения $(1) w\left(z_{1}, z_{2}\right)$ из $\left(\mathscr{E}_{1}\left(\mathscr{F}_{1}\right) ; \mathscr{E}_{2}\left(\mathscr{F}_{2}\right)\right)$ такое, что выполнены соотношения $(2)$, в которых $\varphi_{k}-$ заданные ростки из $\mathscr{E}_{1}\left(\mathscr{F}_{1}\right) \quad(k=$ $0,1, \ldots, m-1)$.

При этом элемент $w\left(z_{1}, z_{2}\right)$ из $\left(\mathscr{E}_{1}\left(\mathscr{F}_{1}\right) ; \mathscr{E}_{2}\left(\mathscr{F}_{2}\right)\right)$ считается решением уравнения (1), если при $l=1,2 \exists T_{l} \in v_{l}\left(\mathscr{F}_{l}\right): w\left(z_{1}, z_{2}\right) \in C^{\infty}\left(T_{l}\right)$ при каждом фиксированном $z_{3-l}$ из $T_{3-l}$ и если правая часть (1) тождественно равна нулю на $T_{1} \times T_{2}$.

Формальное построение решения з.K. (1), (2) производится при тех же исход- 
ных предположениях относительно пространств $\mathscr{E}_{l}\left(\mathscr{F}_{l}\right)$ ростков и так же, как это проделано выше, в $\S 3$, для пространств функций. При этом считаем, что ряд (5) сходится абсолютно в $\left(\mathscr{E}_{1}\left(\mathscr{F}_{1}\right) ; \mathscr{E}_{2}\left(\mathscr{F}_{2}\right)\right)$, если сушествуют множества $T_{l}$ из $v_{l}\left(\mathscr{F}_{l}\right)$ $(l=1,2)$ такие, что при любом фиксированном $z_{l}$ из $T_{l}$ каждый ряд

$$
\sum_{k=1}^{\infty} a_{j, s}^{(k)} z_{2}^{s} \exp \left[\lambda_{k} z_{1}+\mu_{j, k} z_{2}\right], \quad s=0,1, \ldots, p_{j}-1, \quad j=1,2, \ldots, N
$$

сходится абсолютно в $\mathscr{E}_{3-l}\left(\mathscr{F}_{l}\right)$.

Допустим, что ряд (5) сходится абсолютно в $\left(\mathscr{E}_{1}\left(\mathscr{F}_{1}\right) ; \mathscr{E}_{2}\left(\mathscr{F}_{2}\right)\right)$ и что это пространство секвенциально полно. Тогда сумма $w_{0}\left(z_{1}, z_{2}\right)$ ряда $(5)$ принадлежит пространству $\left(\mathscr{E}_{1}\left(\mathscr{F}_{1}\right) ; \mathscr{E}_{2}\left(\mathscr{F}_{2}\right)\right)$. Учитьвая еще, что каждое слагаемое $a_{j, s}^{(k)} z_{2}^{s} \times$ $\exp \left[\lambda_{k} z_{1}+\mu_{j, k} z_{2}\right]$ удовлетворяет уравнению (1) в $\mathbb{C}^{2}$, а оператор дифференцирования непрерьвен в каждом пространстве $\mathscr{E}_{l}\left(\mathscr{F}_{l}\right), l=1,2$, убеждаемся в наличии окрестностей $D_{l}$ из $v_{l}\left(\mathscr{F}_{l}\right)$ таких, что $D_{l} \subseteq T_{l}$ и $w_{0}\left(z_{1}, z_{2}\right) \in C^{\infty}\left(D_{l}\right)$ при любом фиксированном $z_{3-l}$ из $D_{3-l} \quad(l=1,2)$. Кроме того, при $w=w_{0}$ левая часть уравнения (1) тождественно равна нулю на $D_{1} \times D_{2}$. Следовательно, $w_{0}$ - решение уравнения (1) в определенном выше смысле. Далее, $\forall z_{2} \in \mathscr{F}_{2}, \forall z_{1} \in D_{1}$, $\forall n \leqslant m-1$

$$
\frac{\partial^{n} w_{0}\left(z_{1}, z_{2}\right)}{\partial z_{2}^{n}}=\sum_{j=1}^{N} \sum_{s=0}^{p_{j}-1} \sum_{k=1}^{\infty} a_{j, s}^{(k)}\left(z_{2}^{s} \exp \mu_{j, k} z_{2}\right)^{(s)} \exp \lambda_{k} z_{1}
$$

откуда при тех же $n$ и $z_{1}$

$$
\left.\frac{\partial^{n} w_{0}\left(z_{1}, z_{2}\right)}{\partial z_{2}^{n}}\right|_{z_{2}=0}=\sum_{j=1}^{N} \sum_{s=0}^{q_{j}(n)} \sum_{k=1}^{\infty} a_{j, s}^{(k)} s ! C_{n}^{s} \mu_{j, k}^{n-s} \exp \lambda_{k} z_{1}
$$

где, как выше, $\mu_{j, k}=\mu_{j}\left(\lambda_{k}\right), q_{j}(n)=\min \left\{n, p_{j}-1\right\}$.

Как и раньше, показываем, что числа $a_{j, s}^{(k)}$ можно выбрать так, чтобы при любом $k \geqslant 1$ выполнялись соотношения (7), в которых числа $b_{k, n}$ являются коэффициентами разложений $(6)$ начальных ростков $\varphi_{n}$ из $\mathscr{E}_{1}\left(\mathscr{F}_{1}\right)$. При этом справедливы неравенства (9). Во всех тех случаях, когда из абсолютной сходимости рядов (6) можно вьвести с помощью двух неравенств (9) абсолютную сходимость рядов (5) в $\left(\mathscr{E}_{1}\left(\mathscr{F}_{1}\right) ; \mathscr{E}_{2}\left(\mathscr{F}_{2}\right)\right)$, получаем, как вьше, что сумма $w_{0}$ ряда $(5)$ является решением з.К. (1), (2) из $\left(\mathscr{E}_{1}\left(\mathscr{F}_{1}\right) ; \mathscr{E}_{2}\left(\mathscr{F}_{2}\right)\right)$. Далее, предложение 1 остается справедливым и для ПОЛВП $\left(Q ; C^{\infty} ; v(Q)\right)$-ростков (а не функций, как раньше).

Все изложенные после $\S 3$ результаты остаются в силе (с небольшими изменениями в доказательствах и формулировках $)$ и для пространств $\left(Q ; C C^{\infty} ; v(Q)\right)$-ростков. В частности, справедлива теорема 1 и ее следствие, в формулировке которых слово “функций” (теорема 1) и “функции” (следствие) следует заменить на соответственно " $\left(Q ; C^{\infty} ; v(Q)\right)$-ростков" и “ $\left(Q ; C^{\infty} ; v(Q)\right)$-ростки".

Это дает возможность рассмотреть новые примеры пространств $\mathscr{E}_{1}\left(\mathscr{F}_{1}\right)$, кроме имеюшихся в $\S 4$. Приведем один из таких примеров. 
Пусть $\mathscr{K}$ - выпуклый компакт в $\mathbb{C}$ и $\mathscr{H}(\mathscr{K})$ - пространство аналитических ростков на $\mathscr{K}$ с индуктивной топологией

$$
\mathscr{H}(\mathscr{K})=\operatorname{ind}_{n} A C\left(\mathscr{G}_{n}\right),
$$

где $\mathscr{G}_{n}$ - последовательность ограниченных выпуклых областей, убывающих монотонно к $\mathscr{K}$ как к ядру: $\mathscr{G}_{n} \supseteq \mathscr{G}_{n+1} \supseteq \mathscr{K}, \mathscr{K}=\bigcap_{m=1}^{\infty} \mathscr{G}_{m}$, и $A C\left(\mathscr{G}_{n}\right)$ - банахово пространство аналитических в области $\mathscr{G}_{n}$ и непрерывных на $\overline{\mathscr{G}}_{n}$ функций с нормой $\|y\|_{n}:=\max \left\{|y(z)|: z \in \overline{\mathscr{G}_{n}}\right\}$. Как известно [18], $\mathscr{H}(\mathscr{K})-$ регулярный индуктивный предел $B$-пространств $A C\left(\mathscr{G}_{n}\right)$. Kроме того, $\mathscr{H}(\mathscr{K})=\operatorname{ind}_{n} \mathscr{H}\left(\mathscr{G}_{n}\right)$. Из сушествования в $\mathscr{H}\left(\mathscr{G}_{n}\right)$ АПС с описанными в примере 2 из $\S 4$ свойствами нетрудно вывести, что в $\mathscr{H}(\mathscr{K})$ имеется АПС экспонент $\mathscr{E}_{\Lambda}=\left(\exp \lambda_{k} z\right)_{k=1}^{\infty}$, для которой $\lim _{k \rightarrow \infty} \frac{\ln k}{\left|\lambda_{k}\right|}=0$. Более того, если $0 \in \mathscr{K}$ и Int $\mathscr{K} \neq \varnothing$, то можно положить $\mathscr{G}_{n}=\operatorname{Int}\left(q_{n} \mathscr{G}\right)$, где $q_{n} \searrow 1$. Если $\mathscr{E}_{\Lambda}-\mathrm{A \Pi C}$ в $\mathscr{H}\left(\mathscr{G}_{1}\right)$ со свойством $\lim \sup _{k \rightarrow \infty} \frac{k}{\left|\lambda_{k}\right|}<\infty$, то согласно [23] $\mathscr{E}_{\Lambda}-\mathrm{A \Pi C}$ в $\mathscr{H}\left(\mathscr{G}_{n}\right) \forall n \geqslant 1$, и подавно $\mathscr{E}_{\Lambda}-\mathrm{AПC}$ в $\mathscr{H}(\mathscr{K})$. Следовательно, здесь применимы указанные аналоги теоремы 1 и ее следствия.

В частности, если $\mathscr{K}=\{0\}$, то $\mathscr{H}(\mathscr{K})=\overline{A_{0}}-$ пространство всех аналитических ростков в начале координат с обычной индуктивной топологией. Из (обобщенной) теоремы 1 следует, что для любых аналитических в начале координат функций $\varphi_{j}$, $j=0,1, \ldots, m-1$, сушествует решение з.К. (1), (2) из $\left\{\overline{A_{0}}, \mathscr{E}_{2}(\nu, \beta)\right\}$.

Аналогичные примеры можно привести и для значений $\rho_{2}>0$. Опишем один такой пример (см. начало $\S 6$ ). Пусть $\mathscr{G}_{0}$ - ограниченная выпуклая область, содержащая начало координат, и $\mathscr{B}_{0}-$ ее замыкание в $\mathbb{C}$. Положим $\forall \tau \in \mathbb{C}$

$$
\left(\mathscr{B}_{0}\right)_{\tau}=\left\{\left(z_{1}, z_{2}\right) \in \mathbb{C}^{2}: z_{1}+\tau z_{2} \in \mathscr{B}_{0}\right\}
$$

Пусть, как раньше, $\tau_{1}, \tau_{2}, \ldots, \tau_{m}$ - все корни многочлена $P(x)=\sum_{k \in \mathcal{N}_{2}} a_{l_{k}, k} x^{k}$ и $\mathscr{P} \mathscr{B}_{0}=\prod_{k=1}^{m}\left(\mathscr{B}_{0}\right)_{\tau_{k}}$. Легко проверить, что $\mathscr{P} \mathscr{B}_{0}=\overline{\mathscr{P} \mathscr{G}_{0}}$.

Так же, как теорема 6, доказывается

ТЕОРема 24. Если $\varphi_{k}, k=0,1, \ldots, m-1,-$ любые ростки из $\mathscr{H}\left(\mathscr{B}_{0}\right)$, то решение з.К. (1), (2) в $\mathscr{H}\left(\mathscr{P} \mathscr{B}_{0}\right)$ существует. При этом если $\mathscr{E}_{\Lambda}=\left(\exp \lambda_{k} z\right)_{k=1}^{\infty}$ - любая АПС в $\mathscr{H}\left(\mathscr{B}_{0}\right)$, для которой $\lim _{k \rightarrow \infty} \frac{\ln k}{\left|\lambda_{k}\right|}=0$, a (11) - какие-либо разложения (представителей) начальных ростков $\varphi_{k}$ в абсолютно сходящиеся в $\mathscr{H}\left(\mathscr{B}_{0}\right)$ ряды по системе $\mathscr{E}_{\Lambda}$, то в качестве решения з.К. (1), (2) мож-

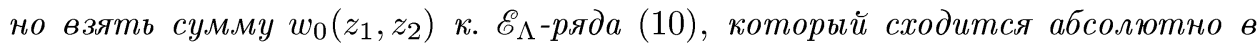
пространстве $\mathscr{H}\left(\mathscr{P} \mathscr{B}_{0}\right)$.

\section{§ 14. Заключительные замечания}

1. Проанализировав исходные предположения, при которых получены вышеизложенные результаты, убеждаемся в том, что фактически была использована непрерывность оператора дифференцирования в пространствах $\mathscr{E}_{l}\left(\mathscr{F}_{l}\right)(l=1,2)$ и наличие в $\mathscr{E}_{1}\left(\mathscr{F}_{1}\right)$ АПС, инвариантной относительно дифференцирования. Но эти 
предположения могут выполняться и для "плохих" (негладких) решений при "плохих" начальных данных - например, при начальных данных, являющихся обобшенными функциями (распределениями). Однако применение изложенного метода решения з.К. в такой ситуации имеет уже ряд особенностей, начиная с перехода от классического решения к обобшенному и заканчивая проверкой наличия АПС с нужными свойствами в пространствах распределений. Так как построение таких (инвариантных относительно дифференцирования) АПС в различных пространствах обощенных функций только начинается, то решение з.К. с помощью АПС в этой ситуации должно быть предметом отдельного исследования.

2. В данной работе показано, что характер гладкости аналитического или ультрадифференцируемого решения з.К. (1), (2) по переменным $z_{1}$ и $z_{2}$ определяется величиной кор. инд. $\rho_{2}$. Следует отметить, что попытки ввести такой параметр, который бы находился эффективно по уравнению (1) и определял сравнительный характер гладкости решения з.К. (1), (2) по переменным $z_{1}$ и $z_{2}$, предпринимались и ранее, в работах Г. С. Салехова и его сотрудников (см. [17], [24], [25]). Однако, на наш взгляд, введенные им параметры типа "веса" оказались не слишком удачными (более подробно этот вопрос проанализирован в $[18, \S 14]$ ).

3. Будем говорить, что уравнение (1) обобщенно нормально по Ковалевской, если оно является уравнением обобщенного класса Ковалевской (см. 1 1), т.е. если $\rho_{2} \leqslant 1$, или, что все равно,

$$
l_{m}+m \geqslant l_{k}+k, \quad k=0,1, \ldots, m-1 \text {. }
$$

В частном случае $l_{m}=0$, т.е. для уравнения (3) условия (35) принимают вид

$$
m \geqslant l_{k}+k, \quad k=0,1, \ldots, m-1 \text {. }
$$

Это условие указано С. В. Ковалевской в ее известном мемуаре от 1875 г., где доказаны существование и единственность решения з.К. (3), (2) в классе голоморфных в окрестности начала функций.

Из результатов $§ 4-6$ следует, что условие (35) обеспечивает сушествование (но не единственность!) голоморфного в окрестности $(0,0)$ решения з.К. (1), (2) для любых голоморфных в окрестности (0) начальных данных. Поэтому это условие можно рассматривать как естественное обобщение на уравнение (1) условия (36) нормальности по Ковалевской уравнения (3). Необходимо, однако, отметить, что в случае, когда $\rho_{2} \leqslant 1$, но $l_{m}>0$, единственности решения з.К. (1), (2)согласно теореме 12 нет даже в очень "узких" подклассах голоморфных решений, и методы С. В. Ковалевской и ее последователей здесь уже неприменимы.

4. Аналитические решения з.К. для различных частных типов уравнения (3) исследовались еще в давних работах Ле Ру, Хольмгрена, Жевре и других (см., например, [26]). В последнее десятилетие появился ряд интересных работ (см., например, [27]-[33]), в которых получены сушественные результаты об аналитических и бесконечно дифференцируемых решениях з.К. для уравнений более общего вида, чем (3), в частности, для уравнений с переменными коэффициентами (но разрешенными относительно старшей производной). Имеются и работы о з.К. для уравнений, не разрешенных относительно производной (см., например, статью [34] 
и библиографию к ней). Однако в этих работах, как правило, з.К. (1), (2) рассматривается в пространстве обобщенных решений, причем $z_{1}=x \in \mathbb{R}^{n}, n \geqslant 1$, $z_{2}=t \geqslant 0$, а сами решения имеют степенной рост по $t$.

Результаты фундаментального характера о з.К. (3), (2) в различных классах обобщенных и классических решений получены И.Г. Петровским и его школой (см. [35] и имеющиеся там обзоры) для случая, когда $z_{2}=t \geqslant 0$, а $z_{1}=x \in \mathbb{R}^{n}$.

Не имея возможности останавливаться здесь на анализе работ [26]-[35] и примыкающих к ним, отметим лишь, что метод данной статьи отличается от методов, использованных в [26]-[35], а ее результаты не следуют из результатов этих работ.

Что же касается метода настояшей статьи, основанного на использовании АПС экспонент, то с его помощью з.К. для уравнений в частных производных с постоянными коэффищиентами и двумя независимыми переменными исследована в работе [14] для общего уравнения второго порядка $(m=2)$, в работах [36], [6] - для некоторых типов уравнения (1) произвольного порядка, отвечающих случаю $\rho_{2}=1$, а в статье [37] - случаю $\rho_{2}<1$. Однако понятие кор. инд., являюшееся центральным в данной работе, появилось только в работах автора [3], [4], [38].

5. Метод данной статьи можно применить и к 3.К. для систем линейных уравнений с зависящими от одномерной переменной $z_{2}$ коэффищиентами и с многомерной независимой переменной $z_{1} \in \mathbb{C}^{p}, p \geqslant 1$. Полученные на этом пути результаты, которые, к сожалению, не имеют такого же законченного характера, как в данной статье, предполагается изложить отдельно.

\section{Список литературы}

1. Мизохата C. Теория уравнений с частными производными. М.: Мир, 1977.

2. Маркушевич А. И. Теория аналитических функций. Т. 2. М.: Наука, 1968.

3. Коробейник Ю. Ф. О периодических решениях задачи Коши. I // Изв. ВУЗов. Математика. 1996. № 3. С. 38-49.

4. Коробейник Ю. Ф. Представляющие системы экспонент и задача Коши для уравнений в частных производных с постоянными коэффициентами. І. Ростов-на-Дону, 1996. Деп. в ВИНИТИ 11.11.96, № 3289-В96.

5. Коробейник Ю. Ф. Представляющие системы // УМН. 1981. Т. 36. №1. С. 73-126.

6. Михайлов А.Б. Об аналитических решениях задачи Коши // Изв. ВУЗов. Северо-Кавказский регион. Естественные науки. 1993. № 3-4. С. 55-60.

7. Напалков В. В. Достаточные множества в одном классе целых функций // Вопросы аппроксимации функций комплексного переменного. Уфа, 1980. С. 110-115.

8. Коробейник Ю.Ф., Абанин А.В. Абсолютно представляющие системы экспонент в пространстве бесконечно дифференцируемых функций и некоторые их применения: Тез. междунар. конференции памяти Н. В. Ефимова. Ростов-на-Дону, 1996. С. 115-116.

9. Леонтьев А. Ф. Ряды экспонент. М.: Наука, 1976.

10. Коробейник Ю. Ф. Представляющие системы // Изв. АН СССР. Сер. матем. 1978. T. 42. № 2. C. $325-355$.

11. Коробейник Ю. Ф. Описание общего вида нетривиальных разложений нуля по экспонентам. Приложения // Изв. АН СССР. Сер. матем. 1991. Т. 55. № 5. С. 1049-1069.

12. Коробейник Ю. Ф. Индуктивные и проективные топологии. Достаточные множества и представляющие системы // Изв. АН СССР. Сер. матем. 1986. Т. 50. № 3. С. 539-565.

13. Епифанов О.В. Вариации слабо достаточных множеств в пространствах аналитических функций // Изв. ВУЗов. Математика. 1986. № 7. С. 50-56.

14. Коробейник Ю. Ф., Михайлов А. Б. Об аналитических решениях задачи Коши // Дифоференц. уравн. 1991. Т. 27. № 3. С. 503-510. 
15. Левин Б. Я. Распределение корней целых функций. М.: ГИТТЛ, 1956.

16. Ткаченко В. А. Уравнения типа свертки в пространствах аналитических функционалов // Изв. АН СССР. Сер. матем. 1977. Т. 41. № 2. С. 378-391.

17. Салехов Г. С. О задаче Коши-Ковалевской для одного класса линейных уравнений с частными производными в области сколь угодно гладких функций // Изв. АН СССР. Сер. матем. 1950. Т. 14. № 4. С. 355-366.

18. Коробейник Ю. Ф. Представляюшие системы экспонент и задача Коши для уравнений в частных производных с постоянными коэффициентами. II. Ростов-на-Дону, 1996. Деп. в ВИНИТИ 11.11.96. № 3290-В96.

19. Хавин В. П. Пространства аналитических функций // Итоги науки. Математический анализ. М.: ВИНИТИ, 1966. С. 76-174.

20. Michael E. Continious Selections. I // Annals of Math. 1956. V. 63. № 2. P. 361-382.

21. Бурбаки Н. Топологические векторные пространства. М.: ИЛ, 1959.

22. Коробейник Ю. Ф., Мелихов $C$. Н. Линейный непрерывный правый обратный для оператора представления и конформные отображения // Докл. РАН 1992. Т. 323. №5. C. $826-829$.

23. Коробейник Ю. Ф., Мелихов $C$. H. Линейный непрерывный правый обратный для оператора представления и приложения к операторам свертки // Сиб. матем. журн. 1993. T. 34. №1. C. $70-84$.

24. Салехов Г. С. К проблеме Коши для линейных уравнений с частными производными в области бесконечно дифференцируемых функций // УМН. 1952. Т. 2. № 2. С. 226-228.

25. Салехов Г. С., Фридлендер В.Г. К вопросу о задаче, обратной задаче Коши-Ковалевской // УМН. 1952. Т. 3. №2. С. 169-192.

26. Holmgren E. Sur l'équation de la propagation de la chaleur // Arkiv för Math. Astr. och Physik. 1908. V. 4. № 1-2, 3-4.

27. Bony J.M., Shapira P. Existence et prolongement des solutions holomorphes des équations aux derivées partielles // Invention Math. 1972. V. 17. P. 95-105.

28. Hamada T., Leray J., Takeuchi A. Prolongment analytique de la solution du problème de Cauchy linéaire // J. de Math. Pures Appl. 1985. V. 64. № 3. P. 257-319.

29. D'Agnolo Andrea. Domain d'existence pour le problème de Cauchy en théorie des faisceaux // J. de Math. Pures Appl. 1993. V. 72. № 1. P. 1-13.

30. Дубинский Ю. А. О корректности задачи Коши в классах целых функций конечного порядка // ДАН СССР. 1988. Т. 301. №6. С. 1305-1308.

31. Дубинский Ю. А. Задача Коши и псевдодифференциальные операторы в комплексной области // УМН. 1990. Т. 45. №2. С. 115-142.

32. Дубинский Ю. А. Задача Коши в комплексной области. М.: Изд-во МЭИ, 1996.

33. Одинцов О.В. Дифференциальные операторы бесконечного порядка в некоторых пространствах целых функций // Изв. РАН. Сер. матем. 1993. Т. 57. № 5. С. 168-185.

34. Павлов А. Л. Задача Коши для уравнения типа Соболева-Гальперна в пространствах функций степенного роста // Матем. сб. 1993. Т. 184. №11. С. 3-20.

35. Петровский И. Г. Избранные труды. Системы уравнений с частными производными. М.: Наука, 1986.

36. Михайлов А. Б., Подпорин В. П. Определение области аналитичности решений задачи Коши // Изв. СКНЦ ВШ. Сер. естествен. наук. 1992. № 1-2. С. 20-24.

37. Коробейник Ю. Ф. Об аналитических решениях задачи Коши для уравнений параболического типа // Дифференц. уравн. 1994. Т. 30. № 10. С. 1774-1781.

38. Коробейник Ю. Ф. О задаче Коши для уравнения в частных производных с двумя независимыми переменными // Алгебра и анализ. Ч. 2. Теория функций и приближений, функциональный анализ, краевые задачи для дифференциальных уравнений в частных производных: Тез. междунар. научной конференции. Казань: Изд-во Казан. ун-та, 1994. C. $75-76$.

Ростовский государственный университет

Поступило в редакцию e-mail: kor@rsu.rnd.su 